\title{
First-Order Phase Transition in Potts Models with finite-range interactions
}

\author{
T. Gobron and I. Merola
}

\begin{abstract}
We consider the $Q$-state Potts model on $\mathbb{Z}^{d}, Q \geq 3, d \geq 2$, with Kac ferromagnetic interactions and scaling parameter $\gamma$. We prove the existence of a first order phase transition for large but finite potential ranges. More precisely we prove that for $\gamma$ small enough there is a value of the temperature at which coexist $Q+1$ Gibbs states. The proof is obtained by a perturbation around mean-field using Pirogov-Sinai theory. The result is valid in particular for $d=2, Q=3$, in contrast with the case of nearest-neighbor interactions for which available results indicate a second order phase transition. Putting both results together provides an example of a system which undergoes a transition from second to first order phase transition by changing only the finite range of the interaction.
\end{abstract}

\section{Introduction}

The Potts model is one of the most studied systems in Statistical Mechanics not to mention its interest in other areas of mathematics and computer sciences. Since its original description by Potts as a simplified version of the clock model [25], it has become an ever growing source of interest, in particular in the field of phase transition. Originally introduced as the simplest generalization of the Ising Model (classical spins with $Q$ values interacting through alike/unlike interactions), it acquired a further significance through the Fortuin-Kasteleyn representation [14, which allows for both a straightforward generalization to any real positive value of the parameter $Q$ (random cluster model [16]-[17]), and a direct connection between its partition function and the Tutte dichromatic polynomial [28]29, which have a central meaning in large areas of graph theory. The FK random cluster representation, gave also rise to important connections with percolation theory $(Q \rightarrow 1)$ and resistor networks $(Q \rightarrow 0)$.

Though the Potts model is in general not solvable, it has been regarded since the original work by Potts as a simple example of an order-disorder phase transition. A lot of work has been dedicated to a rigorous study of the critical properties of the model and their dependence on the number $Q$ of spin values and the dimension $d$ of the lattice. The are exact computations [4, [5] which show that the transition is first order for nearest neighbor interactions in two dimensions when $Q>4$ while it is continuous for $Q \leq 4$, (see $\underline{30}$ for a review), but a complete proof is still missing. A thorough analysis is however available for 
the mean field version of the model where the transition is continuous for $Q \leq 2$ and first order for $Q \geq 3$, independently of the dimensions.

There have been several attempts in various directions to weaken the mean field hypothesis. The idea is to regard the Potts model as a perturbation of its mean field version and this has been achieved in three different regimes: large number of dimensions $d, \mathbf{1 8}$, large number of components $Q, \mathbf{1 2}$, [19], and long range interactions, [1, [7, [8] and the present paper. We also mention that early attempts led to an heuristic determination of a value $Q_{c}(d)$ beyond which the transition becomes mean-field like, and in particular to a few exact results $Q_{c}(2)=4, Q_{c}(4)=2, Q_{c}(6)=1[\mathbf{3 0}]$.

Indeed the most natural way to approximate mean field is to use long range interactions as in [1] where the occurrence of a phase transition is proved in one dimension with an interaction which decays as $1 / r^{2}$. Numerical results, 27, indicate that a transition from continuous to first order occurs for interactions with a power law decay $1 / r^{s}$ when $s$ varies across some $Q$-dependent critical value. More recently $[\mathbf{8}$ have proved for long-range interactions the existence at a special value of the temperature of $Q+1$ distinct DLR states, where $Q$ of them describe ordered phases, each one with a dominant spin, while the last one describes a disordered phase where all spins have same average value. The result applies to special interactions (they should be "reflection positive") and requires slow power law decay in low dimension, $d<3$, while for $d \geq 3$ some exponentially decaying potentials can be also considered.

As already mentioned the present paper is also based on approximating mean field by using long range interactions, but in a sense and with a methodology different from the above papers. We follow the approach proposed by Kac, in particular in its implementation by Lebowitz and Penrose 23. Calling $\gamma$ the scaling parameter of the Kac potential (so that mean field is recovered in the limit $\gamma \rightarrow 0$ ) we will prove that a mean field behavior is observed also at finite $\gamma$ 's, i.e. without taking $\gamma \rightarrow 0$, which, for the potentials that we consider, means that the range of the interaction is strictly finite. More precisely we will show that the coexistence in the mean-field model of $Q+1$ phases at the inverse critical temperature $\beta_{c}^{\mathrm{mf}}$ implies that the same occurs also for finite (and suitably small) values of $\gamma$ but at an inverse temperature $\beta_{c}=\beta_{c}(\gamma)$ which is close to but not necessarily equal to $\beta_{c}^{\mathrm{mf}}$. Moreover in a paper still in preparation we show that the present techniques allow to determine also the structure of the phase diagram around $\beta_{c}(\gamma)$ : there are $\delta$ and $\gamma_{\delta}$ both positive so that for any $\gamma \leq \gamma_{\delta}$ the following holds. When $\beta \in\left(\beta_{c}(\gamma)-\delta, \beta_{c}(\gamma)\right)$ there is a unique extremal, translational invariant [disordered] DLR state while for $\beta \in$ $\left(\beta_{c}(\gamma), \beta_{c}(\gamma)+\delta\right)$ there are exactly $Q$ extremal, translational invariant [ordered] DLR states. This shows that the transition is first order for $Q \geq 3$ and all $d \geq 2$ provided the interaction has a "sufficiently long range", but recall its range is strictly finite.

In some respect, the above results are quite surprising and contradict some deeply rooted beliefs and in particular: 
- All finite range models with the same symmetries and the same dimensions behave the same, in particular nearest neighbors and finite range ferromagnetic bounded spin systems are in a same universality class.

- The Pirogov Sinai theory applies away from the critical point.

- Though a rigorous analysis is still missing, the available results for the $(Q=3, d=$ 2) Potts model with nearest neighbor interaction strongly indicate a second order phase transition [6. In contradistinction, our results show that for a finite but long enough range of interactions, the transition is first order, which in turn suggests that there is a critical interaction range where the transition changes nature from first to second order. If this was to be the case, we would get an example of a modification of the qualitative behavior induced by changing the [finite] range of the interaction and the first item above would be proven false.

- The second statement about Pirogov-Sinai applies to our context because we use extensively the Pirogov-Sinai techniques by "perturbing the mean field ground states". The perturbation is on the inverse interaction range which is 0 in mean field and $\gamma>0$ for the true system. To our knowledge this is the first example where the Pirogov-Sinai theory works in a range which includes the critical point.

The idea of perturbing mean field with Kac potentials is clearly contained in the original papers by Lebowitz and Penrose 23, who introduced a coarse grained description of the model which then plays a fundamental role in the proofs. Using this approach not only as a tool to derive the limit $\gamma \rightarrow 0$ but also in order to study rigorously phase transitions for fixed (small) values of $\gamma>0$ is much more recent, [13] and [10-[1]. The above papers deal with a ferromagnetic Ising systems with Kac potentials and the spin flip symmetry allows to avoid Pirogov-Sinai. Such a symmetry is absent in the models considered in [21], [10] and [2] and a Pirogov-Sinai approach [24] is required, as well as in the Potts model we are considering here. Unfortunately the idea that it is sufficient to take $\gamma$ (instead of the temperature) as the small parameter to get the classical Pirogov-Sinai theory working as well for Kac potentials is a little naive and there is no paper, we believe, in the huge literature on Pirogov-Sinai which covers our case. We thus have to enter into the theory itself and not only check that our model verifies a list of general conditions.

A discussion on the differences with the classical Pirogov-Sinai theory and the techniques used to overcome the corresponding problems has a rather technical nature and does not fit well in an introductory section, so we postpone it to Section 4 where we also outline the scheme of the proofs. We just mention here that an output of the Pirogov-Sinai theory is a control of the local structure of the phase diagram. The work however becomes much simpler if less ambitiously we restrict to the problem of finding a temperature at which the $Q+1$ phases coexist. This does not require to determine the landscape of the metastable free energies of the $Q+1$ phases, but only the existence of a temperature where they are all equal. This is what we do here, the result as it stands, is indeed compatible with the existence of many other nearby temperatures where the same happens. In the 
forthcomming paper mentioned previously, we will exclude such possibilities with an argument which extends $\mathbf{9}$ avoiding the analysis of metastable free energies landscape and allows to characterize all the ergodic DLR states.

The paper is organized as follows: In Section 2, we define the model and state our main result. In section 3, we introduce the scales which appear in the problem and define the contours we will deal with. In Section 4, we explain the strategy of the proofs and introduce abstract contours models which are at the core of the Pirogov-Sinai theory in the Zahradnik approach, 31. In section 5, we define the coarse-grained configurations and prove a "Lebowitz Penrose theorem", introducing a mean field functional. In section 6 we identify the value of the inverse temperature $\beta$ at which the order-disorder phase transition takes place. Section 7 contains an estimate for the finite volume corrections to the pressure, which requires both a control on the decay of correlation and a small deviation estimate. In Section 8, we prove an approximate factorization theorem for the contour weights stated in section 4 and derive the large deviation estimate which provides the Peierls constant.

Six appendices are added at the end of the paper: Appendix A contains a short review of the mean field theory for the Potts model as well as the derivation of the properties on which we rely in the rest of the paper. Appendix B discuss the local equilibrium properties which are used in various parts of the paper. In Appendix C, we prove the existence of the pressure for the abstract models introduced in section 6. In Appendix D and E, we prove two lemmas needed in section 7. Finally, in Appendix E, we give the proof of theorem 6.1]

\section{Model and main results.}

Two equivalent representations: The $Q$-state Potts model on $\mathbb{Z}^{d}, Q>2$ an integer, may be equivalently regarded as a system of classical spins which take $Q$ values, called "colors", or else as a system of $Q$ species of particles with the constraint that at each site there is one and only one particle. In the sequel, we will rather stick to the second interpretation since its implementation fits better both with the coarse-graining we need to consider, and with the mean-field free energy functional to be introduced later.

In the first interpretation we call $\sigma(i)$ the spin at site $i \in \mathbb{Z}^{d}, \tilde{\Omega}_{0}:=\left\{a_{1}, \ldots a_{Q}\right\}$, the set of "colors" the spins take value in, $\tilde{\Omega}:=\tilde{\Omega}_{o}^{\mathbb{Z}^{d}}$, the configuration space, and $\tilde{\Omega}_{\Lambda}:=\tilde{\Omega}_{o}^{\Lambda}$ its restriction to a finite subset $\Lambda$ of $\mathbb{Z}^{d}$.

In the second interpretation $\xi_{q}(i)$ denotes the occupation number at site $i \in \mathbb{Z}^{d}$ of the species $q \in Q$. Let $\Omega_{0}:=\left\{\vec{u}_{1}, \cdots, \vec{u}_{Q}\right\}$ be the set of unit vectors in $\mathbb{R}^{Q}$ with components

$u_{q, k}=\delta_{q, k}$. Due to the constraint $\sum_{q \in Q} \xi_{q}(i)=1$, the collection of all occupation numbers at 
site $i$ can be written as a density vector, $\vec{\xi}(i)=\left(\xi_{q}(i)\right)_{q \in Q}$, taking value in $\Omega_{o}$. We denote the configuration space as $\Omega:=\Omega_{o}^{\mathbb{Z}^{d}}$.

There is obviously a one to one correspondence between $\Omega$ and $\tilde{\Omega}$, defined by associating to each element $\sigma$ of $\tilde{\Omega}$, a vector configuration $\vec{\xi}$ of $\Omega$ as

$$
\vec{\xi}(i)=\vec{u}_{q} \Longleftrightarrow \sigma(i)=a_{q}
$$

Kac potentials: Denoting by $\gamma>0$ a "scaling parameter", let $J_{\gamma}$ be the kernel defined on $\mathbb{R}^{d} \times \mathbb{R}^{d}$ as

$$
J_{\gamma}(x, y)=\gamma^{d} \mathcal{J}(\gamma(x-y))
$$

where $\mathcal{J}(r)$ is a spherically symmetric probability density supported by the unit ball and differentiable with bounded derivative.

Then the Potts-Kac energy in a finite region $\Lambda$ with boundary conditions $\sigma_{\Lambda^{c}} \in \tilde{\Omega}_{\Lambda^{c}}$ is

$$
H_{\gamma, \Lambda}\left(\sigma_{\Lambda} \mid \sigma_{\Lambda^{c}}\right):=-\frac{1}{2} \sum_{\substack{i, j \in \Lambda \\ i \neq j}} J_{\gamma}(i, j) \mathbf{1}_{\left\{\sigma_{\Lambda}(i)=\sigma_{\Lambda}(j)\right\}}-\sum_{\substack{i \in \Lambda \\ j \in \Lambda^{c}}} J_{\gamma}(i, j) \mathbf{1}_{\left\{\sigma_{\Lambda}(i)=\sigma_{\Lambda^{c}}(j)\right\}}
$$

which, in the particle representation, reads

$$
H_{\gamma, \Lambda}\left(\vec{\xi}_{\Lambda} \mid \vec{s}_{\Lambda^{c}}\right)=-\frac{1}{2} \sum_{\substack{i, j \in \Lambda \\ i \neq j}} J_{\gamma}(i, j) \vec{\xi}_{\Lambda}(i) \cdot \vec{\xi}_{\Lambda}(j)-\sum_{\substack{i \in \Lambda \\ j \in \Lambda^{c}}} J_{\gamma}(i, j) \vec{\xi}_{\Lambda}(i) \cdot \vec{s}_{\Lambda^{c}}(j)
$$

where the characteristic functions in (2.3) have been substituted by a scalar product between density vectors, $\vec{\xi} \cdot \vec{\xi}^{\prime}:=\sum_{q=1}^{Q} \xi_{q} \xi_{q}^{\prime}$. This representation allows in particular to extend the definition (2.4) to a wider set of boundary conditions $\vec{s}_{\Lambda^{c}}$, where $\vec{s}$ is taken in $L^{\infty}\left(\mathbb{Z}^{d}, S_{Q}\right)$, with $S_{Q}$ is the set of all density vectors in $\mathbb{R}^{Q}$,

$$
S_{Q}=\left\{\vec{\rho} \in \mathbb{R}_{+}^{Q}, \sum_{q} \rho_{q}=1\right\}
$$

The set $S_{Q}$ is the convex set in $\mathbb{R}^{Q}$ which extremal points identify with the elements of $\Omega_{O}$ :

The finite-volume Gibbs specifications are then the probability measures

$$
\mu_{\gamma, \beta, \Lambda}\left(\vec{\xi}_{\Lambda} \mid \vec{s}_{\Lambda^{c}}\right)=\frac{e^{-\beta H_{\gamma, \Lambda}\left(\vec{\xi}_{\Lambda} \mid \vec{s}_{\Lambda^{c}}\right)}}{Z_{\gamma, \beta, \Lambda}\left(\vec{s}_{\Lambda^{c}}\right)}
$$

where $Z_{\gamma, \beta, \Lambda}\left(\vec{s}_{\Lambda^{c}}\right)$ is the partition function

$$
Z_{\gamma, \beta, \Lambda}\left(\vec{s}_{\Lambda^{c}}\right):=\sum_{\vec{\xi}_{\Lambda}} e^{-\beta H_{\gamma, \Lambda}\left(\vec{\xi}_{\Lambda} \mid \vec{s}_{\Lambda^{c}}\right)}
$$


Mean field: The mean field free energy density is

$$
\phi_{\beta}^{\operatorname{mf}}(\vec{\rho})=-\frac{1}{2} \sum_{q=1}^{Q} \rho_{q}^{2}+\frac{1}{\beta} \sum_{q=1}^{Q} \rho_{q} \ln \left(\rho_{q}\right), \quad \vec{\rho}=\left(\rho_{q}\right)_{q \in Q} \in S_{Q}
$$

The $q$-th component $\rho_{q}$ is interpreted as the density of particles of species $q$, the first term in (2.7) is then (at leading order in the number of particles) the energy density supposing that each particle interacts equally with all the others, and the second term is the entropy. Referring to Appendix $\mathrm{A}$ for details, we recall that in the mean field theory, for each value of $Q$, there is a critical inverse temperature $\beta_{c}^{\mathrm{mf}}$ such that:

for all $\beta<\beta_{c}^{\mathrm{mf}}, \phi_{\beta}^{\mathrm{mf}}$ has a unique minimizer denoted by $\vec{\rho}_{\beta}^{-1}$;

for all $\beta>\beta_{c}^{\text {mf }}$ there are $Q$ minimizers $\vec{\rho}_{\beta}^{p}, p \in\{1, \cdots, Q\}$;

for $\beta=\beta_{c}^{\mathrm{mf}}$ there are $Q+1$ minimizers $\vec{\rho}_{c}^{\hat{p}}, \hat{p} \in\{-1,1, \cdots, Q\}$.

In the above result and in the sequel, we label with $\hat{p}, \hat{p} \in\{-1,1, \cdots, Q\}$, the $Q+1$ mean field minimizers at the critical temperature, $\hat{p}=-1$ referring to the disordered phase, and $\hat{p}=q, q>0$ the ordered one in which the color $q$ dominates.

Main result: For the finite range Kac-Potts models with $\gamma$ small enough, a situation similar to the mean-field results holds. Calling a set of DLR measures mutually independent if none of them is a convex combination of the others, we will prove in the sequel the following:

Theorem 2.1. For any $d \geq 2$ and $Q \geq 3$, there exists $\bar{\gamma}>0$ such that for any $\gamma \in(0, \bar{\gamma})$, there is a value $\beta=\beta_{c}(\gamma)$ at which there are $Q+1$ mutually independent $D L R$ measures with Gibbs specifications (2.6) $, \mu_{\gamma, \beta_{c}(\gamma)}^{\hat{p}}, \hat{p}=-1,1, \cdots, Q$.

In the course of the proof, we will characterize quite explicitly the support properties of the DLR measures $\mu_{\gamma, \beta_{c}(\gamma)}^{\hat{p}}$ which will make evident closeness to mean field, in particular we will see that for a suitable constant $c$

$$
\left|\beta_{c}(\gamma)-\beta_{c}^{\mathrm{mf}}\right|<c \gamma^{1 / 2}
$$

and prove that with large probability in $\mu_{\gamma, \beta_{c}(\gamma)}^{\hat{p}}$ the empirical average of $\vec{\xi}(i)$ over suitably large blocks is close to the (critical) mean field value $\vec{\rho}_{c}^{\hat{p}}$.

In a forthcoming paper we will also prove that the DLR measures $\mu_{\gamma, \beta_{c}(\gamma)}^{\hat{p}}$ are translational invariant and have trivial $\sigma$-algebra at infinity; moreover any other translational invariant DLR measure is a convex combination of the $\mu_{\gamma, \beta_{c}(\gamma)}^{\hat{p}}$ which are then the only ergodic DLR measures. 


\section{Scales, Phase Indicators and Contours}

section3 Coarse graining is the master word in the proof of Theorem 2.1. We will need to define three scale lengths $\ell_{0}, \ell_{-, \gamma}$ and $\ell_{+, \gamma}$, depending on the scale parameter $\gamma$. The first one, the shortest, will be used to estimate partition functions à la Lebowitz-Penrose.

$\ell_{0}$ is much shorter than the interaction range $\gamma^{-1}$, yet much larger than the lattice spacing, set equal to $1 . \ell_{-, \gamma}$ is the scale at which one "recognizes a phase": the empirical average of the spins in boxes of side $\ell_{-, \gamma}$ will be used as an indicator of the local state of the system. When compared to the mean field equilibrium value it will allow us to check whether the system is locally close to an equilibrium. $\ell_{-, \gamma}$ will be chosen much larger than $\ell_{0}$, yet still much smaller than the range $\gamma^{-1}$ so that the indicator can be regarded as a truly local estimator. Finally $\ell_{+, \gamma}$ is chosen much larger than $\gamma^{-1}$, such that if in a box of side $\ell_{+, \gamma}$ the phase indicator constantly indicates the same equilibrium, then the behavior of the spins inside the box but far from the boundaries is almost uncorrelated to the outside.

A possible choice for $\ell_{0}, \ell_{-, \gamma}$ and $\ell_{+, \gamma}$ is to have them scale as $\gamma^{-\frac{1}{2}}, \gamma^{-(1-\alpha)}$ and $\gamma^{-(1+\alpha)}$, with $\alpha$ positive and small enough. More precisely we set these three lengthes as the closest powers of 2 to these values

$$
\ell_{0}=2^{\left[\frac{1}{2} \frac{\ln \gamma^{-1}}{\ln 2}\right]}, \quad \ell_{ \pm, \gamma}=2^{\left[(1 \pm \alpha) \frac{\ln \gamma^{-1}}{\ln 2}\right]}
$$

([.] is the integer part of $\cdot)$, so that the ratios $\frac{\ell_{+, \gamma}}{\ell_{-, \gamma}}$ and $\frac{\ell_{-, \gamma}}{\ell_{0}}$ are integers. We can then construct three partitions $\mathcal{D}^{\ell}$ of $\mathbb{R}^{d}$ in cubes of size $\ell, \ell=\ell_{0}, \ell_{-, \gamma}, \ell_{+, \gamma}$ which are one coarser than the other (if $\gamma$ is small enough). In order to define our local phase indicator,we need to define an accuracy parameter $a$. In the course of the proof, various restrictions on the possible choices of $\alpha$ and $a$ will appear, none of which critical, nor necessarily optimal. We write them here for the reader's convenience, but it is somewhat simpler to keep in mind the choice $\alpha<<1$ and $a<<\alpha$. In the sequel, we will require:

$$
\begin{aligned}
\alpha & <\frac{1}{16 d} \\
a & <\min \left(\frac{1}{4}, \frac{\alpha}{2}\right) \\
-d(1-\alpha)+2 a & <-2 d \alpha
\end{aligned}
$$

We define a local phase indicator as

$$
\eta_{x}(\vec{\xi}):= \begin{cases}\hat{p} \quad \text { if }\left\|\vec{\xi}^{\ell_{-, \gamma}}(x)-\vec{\rho}_{c}^{\hat{p}}\right\|_{\star}<\gamma^{a}, \quad \hat{p} \in\{-1,1, \ldots, Q\} \\ 0 \quad \text { otherwise. }\end{cases}
$$

where $\|\cdot\|_{\star}$ is the sup norm, $\vec{\rho}_{c}^{\hat{p}}$ is the $\hat{p}$-th minimizer of the mean field free energy functional at $\beta=\beta_{c}^{\text {mf }}$ and $\vec{\xi}^{\ell}(x)$ is the empirical average of $\vec{\xi}$ over $C_{x}^{\ell}$, the cube of the partition $\mathcal{D}^{\ell}$ which contains $x$ :

$$
\vec{\xi}^{\ell}(x)=\ell^{-d} \sum_{i \in C_{x}^{\ell}} \vec{\xi}(i)
$$


We also define a phase indicator at scale $\ell_{+, \gamma}$,

$$
\Theta_{x}(\vec{\xi})= \begin{cases}\eta_{x}(\vec{\xi}) & \text { if } \eta_{y}(\vec{\xi})=\hat{p} \quad \forall y: C_{y}^{\ell_{+}, \gamma} \sim C_{x}^{\ell_{+}, \gamma}, \\ 0 & \text { otherwise. }\end{cases}
$$

The set $\left\{y: C_{y}^{\ell_{+}, \gamma} \sim C_{x}^{\ell_{+, \gamma}}\right\}$ is the set of cubes in $\mathcal{D}^{\ell_{+}, \gamma} *$-connected with $C_{x}^{\ell_{+, \gamma}}$, i.e. : $\overline{C_{y}^{\ell+, \gamma}} \sqcap \overline{C_{x}^{\ell+, \gamma}} \neq \emptyset$.

By definition, for any $\hat{p} \neq \hat{q}$, the distance between any regions $\left\{x: \Theta_{x}(\vec{s})=a_{\hat{p}}\right\}$ and $\left\{x: \Theta_{x}(\vec{s})=a_{\hat{q}}\right\}$ is at least $2 \ell_{+, \gamma}$. The interspace between these regions will be the support for the contours which we now define with respect to a configurations $\vec{\xi} \in \Omega$. A similar definition will also hold with respect to a continuous profile $\vec{\rho} \in L^{\infty}\left(\mathbb{R}^{d}, S_{Q}\right)$.

Definition 3.1. A contour $\Gamma \equiv\left(\operatorname{sp}(\Gamma), \eta_{\Gamma}\right)$ for a configuration $\vec{\xi}$ in $\Omega$, is specified by a couple $\left(\operatorname{sp}(\Gamma), \eta_{\Gamma}\right)$ where $\operatorname{sp}(\Gamma)$ is one of the maximal connected component of the subset $\left\{x: \Theta_{x}(\vec{\xi})=0\right\}$ and $\eta_{\Gamma}$ is the coarse grained configuration on $\operatorname{sp}(\Gamma)$ at scale $\ell_{\gamma,-}$ : $\eta_{\Gamma} \equiv\left\{\eta_{x}(\vec{\xi})\right\}_{x \in \operatorname{sp}(\Gamma)}$.

We now define the weight of these contours in the following way:

We denote by $|\Gamma| \equiv|\operatorname{sp}(\Gamma)|$ the volume of the region $\operatorname{sp}(\Gamma)$, and by $N_{\Gamma}:=\frac{|\Gamma|}{\left|C_{0}^{\ell+, \gamma}\right|}$ the number of $\mathcal{D}^{\ell_{+, \gamma}}$-cubes in $\operatorname{sp}(\Gamma)$. For any bounded contour $(|\Gamma|<\infty)$, we denote by $\operatorname{Ext}(\Gamma)$ the (unique) unbounded connected component of $\operatorname{sp}(\Gamma)^{c}$ and by $\left\{\operatorname{Int}_{i}(\Gamma)\right\}, i \in I$ the collection of its bounded connected components.

For any set $B \sqsubset \mathbb{R}^{d} B \sqsubset \mathbb{R}^{d}, r \in \mathbb{R}$, we define

$$
\begin{aligned}
\delta_{\text {in }}^{r}[B] & :=\left\{x \in B: \operatorname{dist}\left(x, B^{c}\right) \leq r\right\} \\
\delta_{\text {out }}^{r}[B] & :=\left\{x \in B^{c}: \operatorname{dist}(x, B) \leq r\right\}
\end{aligned}
$$

where $\operatorname{dist}(x, B)=\inf _{y \in B} \operatorname{dist}(x, y)$ and $\operatorname{dist}(x, y)=\sup _{1 \leq k \leq d}\left|x_{k}-y_{k}\right|$.

Now, for any point $x$ in $\delta_{\text {out }}^{\ell+\gamma}[\operatorname{sp}(\Gamma)], \Theta_{x} \neq 0$ and its value is fixed by $\eta_{\Gamma}$. We define

$$
\begin{aligned}
A^{\hat{q}} \equiv A^{\hat{q}}(\Gamma):=\left\{x \in \delta_{\text {out }}^{\ell+, \gamma}[\operatorname{sp}(\Gamma)]: \Theta_{x}=a_{q}\right\} & ; \quad A(\Gamma):=\bigsqcup_{\hat{q}} A^{\hat{q}}=\delta_{\text {out }}^{\ell+, \gamma}[\operatorname{sp}(\Gamma)] \\
I^{\hat{q}}(\Gamma):=\left\{i: \operatorname{Int}_{i}(\Gamma) \sqcap A^{\hat{q}} \neq \emptyset ;\right\} & ; \quad \operatorname{Int}^{\hat{q}}(\Gamma):=\bigsqcup_{i \in I^{\hat{q}}(\Gamma)} \operatorname{Int}_{i}(\Gamma)
\end{aligned}
$$

We call $\Gamma$ a " $\hat{p}$-contour" if $\operatorname{Ext}(\Gamma) \sqcap A^{\hat{p}} \neq \emptyset$ and define

$$
c(\Gamma):=\operatorname{sp}(\Gamma) \sqcup_{i \in I} \operatorname{Int}_{i}(\Gamma)
$$

Let denote by $\mathcal{E}(\Gamma, \hat{p})$ the event that $\Gamma$ is a $\hat{p}$-contour:

$$
\mathcal{E}(\Gamma, \hat{p}):=\left\{\vec{\xi}: \eta_{x}(\vec{\xi})=\eta_{\Gamma} \quad \forall x \in \operatorname{sp}(\Gamma) \quad ; \begin{array}{c}
\Theta_{x}(\vec{\xi})=a_{\hat{q}} \quad \forall x \in A^{\hat{q}}(\Gamma) \quad \forall \hat{q} \in\{-1,1, \ldots, Q\} \\
\Theta_{x}(\vec{\xi})=a_{\hat{p}} \quad \forall x \in A(\Gamma) \sqcap \operatorname{Ext}(\Gamma)
\end{array}\right\}
$$


and by $\mathcal{E}(\not, \hat{p})$ the event that the phase $\hat{p}$ extends on $\operatorname{sp}(\Gamma) \sqcup A(\Gamma)$

$$
\mathcal{E}(X, \hat{p}):=\left\{\vec{\xi}: \Theta_{x}(\vec{\xi})=a_{\hat{p}} \quad \forall x \in \operatorname{sp}(\Gamma) \sqcup A(\Gamma)\right\}
$$

We then define the weight of a $\hat{p}$-contour as the ratio:

$$
w_{\gamma, \beta}^{\hat{p}}\left(\Gamma ; \vec{\xi}_{A^{\hat{p}}}\right):=\frac{\mu_{\gamma, \beta, c(\Gamma) \backslash \operatorname{Int}^{\hat{p}}(\Gamma)}\left(\left.\mathcal{E}(\Gamma, \hat{p})\right|_{\vec{\xi}_{A^{\hat{p}}}}\right)}{\mu_{\gamma, \beta, c(\Gamma) \backslash \operatorname{Int}^{\hat{p}}(\Gamma)}\left(\mathcal{E}(\Gamma, \hat{p}) \mid \vec{\xi}_{A^{\hat{p}}}\right)}
$$

Let $\mathcal{X}^{\hat{p}}$ be the set of "correct" $\hat{p}$-configurations:

$$
\mathcal{X}^{\hat{p}}:=\left\{\vec{s}: \Theta_{x}(\vec{s})=a_{\hat{p}} \forall x \in \mathbb{R}^{d}\right\}
$$

where, depending on the context, $\vec{s}$ will be either in $\Omega$ or in $L^{\infty}\left(\mathbb{Z}^{d}, S_{Q}\right)$

Using an iteration procedure, the partition function with boundary conditions in $\mathcal{X}^{\hat{p}}$ can be rewritten in terms of $\hat{p}$-contours as:

$$
Z_{\gamma, \beta, \Lambda}^{\hat{p}}\left(\vec{s}_{\Lambda^{c}}\right)=\sum_{\underline{\Gamma} \in \mathcal{B}_{\Lambda}^{\hat{p}}} \sum_{\vec{\xi}_{\Lambda} \in \mathcal{X}_{\Lambda}^{\hat{p}}} \prod_{\Gamma \in \underline{\Gamma}} w_{\gamma, \beta}^{\hat{p}}\left(\Gamma ; \vec{\xi}_{A^{\hat{p}}}\right) e^{-\beta H_{\gamma, \Lambda}\left(\vec{\xi}_{\Lambda} \mid \vec{s}_{\Lambda^{c}}\right)}
$$

where $\underline{\Gamma}$ is a configuration of compatible $p$-contours and $\mathcal{B}_{\Lambda}^{\hat{p}}$ is a the set of all possible configurations of compatible $\hat{p}$-contours with support inside $\Lambda$.

The expression (3.19) already shows some of the main differences we incounter here with respect to the classical Pirogov-Sinai theory at low temperature.

- The "ground configurations" $\mathcal{X}^{\hat{p}}, \hat{p}=-1,1, \ldots, Q$ are not fixed configurations, but ensemble of configurations. The study of these configurations and of their perturbations is then more complicated and involve the study of variational problem for a non local functional.

- The partition function cannot be express in terms of p-compatible contours because it persists a weak interaction between contours

A similar situation appear in [10-11], where Kac models are considered at low temperature. For such models, the references configurations cannot be chosen as the ground states of the energy even at low temperatures, because the direct interaction between two spin is too weak, an one needs to take into account the local entropy. The partition function is then expressed in terms of interacting contours.

Nevertheless the techniques developed in 10-11, cannot be immediately applied here, since they are based on a cluster expansion at low temperatures in order to extend the classical PS theory to the case of weak interactions, giving a result uniform in the range of the interaction.

Here, we will follow the extension of Pirogov-Sinai techniques to high temperatures used in 22 and $[\mathbf{2 6}$ which also deal with perturbation of a mean-field theory. 


\section{Main steps of the proof}

In this section we state, postponing the proofs, the main steps that lead to the proof of the Theorem 2.1

A very preliminary step is an approximate factorization of the contours weights (3.17), which relies on properties of the following mean-field free energy functional defined on the functions in $\vec{\rho} \in L^{\infty}\left(\Lambda, S_{Q}\right)$ as follows:

$$
F_{\gamma, \beta, \Lambda}\left(\vec{\rho}_{\Lambda} \mid \vec{\rho}_{\Lambda^{c}}\right)=V_{\gamma, \Lambda}\left(\vec{\rho}_{\Lambda} \mid \vec{\rho}_{\Lambda^{c}}\right)-\frac{1}{\beta} I\left(\vec{\rho}_{\Lambda}\right)
$$

where $\vec{\rho}_{\Lambda^{c}} \in L^{\infty}\left(\Lambda^{c}, S_{Q}\right)$ defines the boundary conditions. The two functionals $V_{\gamma, \Lambda}\left(\vec{\rho}_{\Lambda} \mid \vec{\rho}_{\Lambda^{c}}\right)$ and $I\left(\vec{\rho}_{\Lambda}\right)$ are respectively the energy and the entropy of $\vec{\rho}_{\Lambda}$,

$$
\begin{gathered}
V_{\gamma, \Lambda}\left(\vec{\rho}_{\Lambda} \mid \vec{\rho}_{\Lambda^{c}}\right)=-\frac{1}{2} \int_{\Lambda} \int_{\Lambda} J_{\gamma}(x, y)\left(\vec{\rho}_{\Lambda}(x) \cdot \vec{\rho}_{\Lambda}(y)\right) d x d y-\int_{\Lambda} \int_{\Lambda^{c}} J_{\gamma}(x, y)\left(\vec{\rho}_{\Lambda}(x) \cdot \vec{\rho}_{\Lambda^{c}}(y)\right) d x d y \\
I\left(\vec{\rho}_{\Lambda}\right)=-\int_{\Lambda} \sum_{q=1}^{Q} \rho_{\Lambda, q}(x) \ln \left(\rho_{\Lambda, q}(x)\right) d x
\end{gathered}
$$

These functionals comes out naturally after a coarse graining procedure on the scale $\ell_{0}$. In particular, we will prove the following

Proposition 4.1. There exists a constant $c>0$ such that for all $\gamma>0$ and all bounded $\mathcal{D}^{\ell_{0}}$-measurable regions $\Lambda$ in $\mathbb{R}^{d}$,

$$
\left|\log Z_{\gamma, \beta, \Lambda}\left(\vec{\xi}_{\Lambda^{c}}\right)+\beta \inf _{\vec{\rho}_{\Lambda}} F_{\gamma, \beta, \Lambda}\left(\vec{\rho}_{\Lambda} \mid \vec{\xi}_{\Lambda^{c}}^{\ell_{0}}\right)\right| \leq \beta c \gamma^{\frac{1}{2}}|\Lambda|
$$

This result together with a local stability result for the functional around its minimizers allows us to set $\vec{\rho}_{c}^{\hat{q}}$ (respectively $\vec{\rho}_{c}^{\hat{p}}$ ) on $A^{\hat{q}}, \hat{q} \neq \hat{p}$, in the expression of the numerator (respectively denominator) of the left hand side of (3.17) at the price of a small error and get the following bound:

Theorem 4.2. There are $\bar{\gamma}, \bar{b}$ and a constant $c$ such that for all $\gamma<\bar{\gamma}$, $\left|\beta-\beta_{c}^{\mathrm{mf}}\right|<\bar{b}:$

$$
\begin{aligned}
& w_{\gamma, \beta}^{\hat{p}}\left(\Gamma ; \vec{\xi}_{A^{\hat{p}}}\right) \leq \prod_{\hat{q} \neq \hat{p}} \frac{Z_{\gamma, \beta, \operatorname{Int}}^{\hat{q}}(\Gamma) \backslash A^{\hat{q}}\left(\hat{\rho}^{\hat{q}}\right)}{Z_{\gamma, \beta, \operatorname{Int}^{\hat{q}}(\Gamma) \backslash A^{\hat{q}}}^{\hat{\rho}}\left(\vec{\rho}^{\hat{p}}\right)} \exp \left\{-\beta\left|A^{\hat{q}}\right|\left(\phi_{\beta}^{\operatorname{mf}}\left(\vec{\rho}^{\hat{q}}\right)-\phi_{\beta}^{\operatorname{mf}}\left(\vec{\rho}^{\hat{p}}\right)\right)\right\}
\end{aligned}
$$

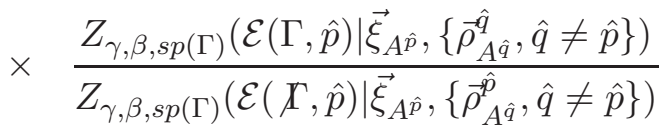

$$
\begin{aligned}
& \times \quad \exp \left\{c \gamma^{\frac{1}{2}}|\Gamma|\right\}
\end{aligned}
$$

Provided a good control on the ratios of partition functions in the first line of (4.2), the factor in the second line will provide the Peierls bound, using a large deviation result for the functional $F_{\gamma, \beta}$ on $\operatorname{sp}(\Gamma)$ and paying again a price of order $\exp \left\{c \gamma^{\frac{1}{2}}|\Gamma|\right\}$. This will finally lead to the the following Theorem: 
Theorem 4.3. There is $\bar{\gamma}$ such that for any $\gamma<\bar{\gamma}$ there exists $\beta_{c}(\gamma)$, such that $\forall \hat{q}$ :

$$
w_{\gamma, \beta_{c}(\gamma)}^{\hat{q}}\left(\Gamma ; \vec{\xi}_{A^{\hat{q}}}\right) \leq \exp \left\{-\mathfrak{K}_{\gamma}\left|N_{\Gamma}\right|\right\} \quad \text { with } \quad \mathfrak{K}_{\gamma}=c_{f} \gamma^{2 a}\left|C^{\ell_{-, \gamma}}\right|
$$

with $c_{f}$ a constant depending only on $d$ and $Q$,

$$
c_{f}=\frac{1}{3^{d+1}}\left(Q-\beta_{c}^{\mathrm{mf}}\right)>0
$$

The proof of the Theorem 2.1 will follow immediately from the above, using the well known Peierls argument that will be omitted here.

Now the main work is to obtain a good control on the ratios

$$
\frac{Z_{\gamma, \beta, \Delta}^{\hat{q}}\left(\vec{\rho}^{\hat{q}}\right)}{Z_{\gamma, \beta, \Delta}^{\hat{p}}\left(\vec{\rho}^{\hat{p}}\right)}
$$

Of course, whenever both $\hat{p}$ and $\hat{q}$ refer to ordered states, the ratio is equal to one by symmetry (by permutation of the colors), and we need essentially to consider the case when $\hat{p}$ or $\hat{q}$ equals -1 . Here we need the Pirogov-Sinai theory and we follow the Zahradnìk's approach. We introduce $Q+1$ "abstract contour models" defined on the product spaces: $\mathcal{X}^{\hat{p}} \times \mathcal{B}^{\hat{p}}$. The partition function of the $\hat{p}$-th abstract model and the weights of the contours are defined recursively as:

$$
Z_{\mathrm{abs}, \beta, \Lambda}^{\hat{p}}\left(\vec{s}_{\Lambda^{c}}\right)=\sum_{\underline{\Gamma} \in \mathcal{B}_{\Lambda}^{\hat{p}}} \sum_{\vec{\xi}_{\Lambda} \in \mathcal{X}_{\Lambda}^{\hat{p}}} \prod_{\Gamma \in \underline{\Gamma}} W^{\hat{p}}(\Gamma ; \vec{\xi}) e^{-\beta H_{\gamma, \Lambda}\left(\vec{\xi}_{\Lambda} \mid \vec{s}_{\Lambda^{c}}\right)}
$$

and

$$
\hat{W}_{\gamma}^{\hat{p}}(\Gamma ; \vec{s}):=\min \left\{w_{\mathrm{abs}, \beta}^{\hat{p}}(\Gamma ; \vec{s}), e^{-\frac{\mathfrak{k}_{\gamma}}{2} N_{\Gamma}}\right\}
$$

where $\mathfrak{K}_{\gamma}$ is given by (4.3) and $w_{\mathrm{abs}, \beta}^{\hat{p}}(\Gamma ; \vec{s})$ is given by

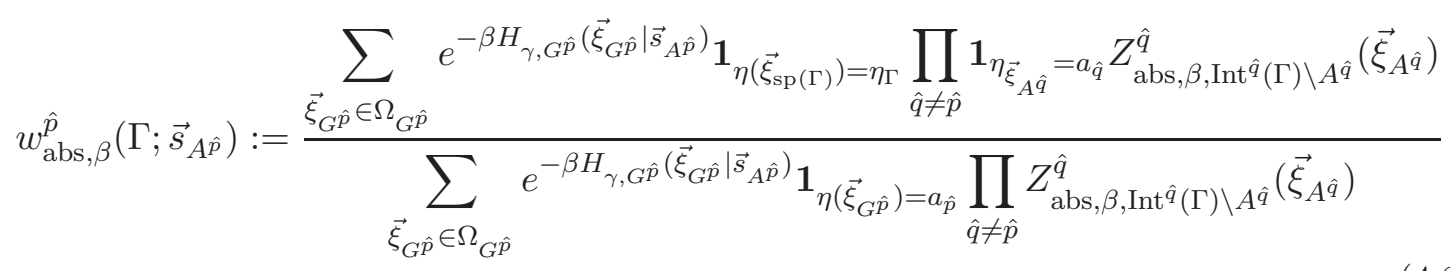

where $G^{\hat{p}}=\operatorname{sp}(\Gamma) \bigsqcup_{\hat{q} \neq \hat{p}} A^{\hat{q}}$.

We stress that the elements of a pair $(\vec{\xi}, \underline{\Gamma})$ in an abstract model are totally unrelated, in fact the configuration $\vec{\xi}$ is in $\mathcal{X}^{\hat{p}}$ and therefore has no contours. The sum $\sum_{\vec{\xi}_{G \hat{p}} \in \Omega_{G \hat{p}}}$ appearing in the definition (4.8) enters only as definition of the weights, without any relations with the configurations $\vec{\xi} \in \mathcal{X}^{\hat{p}}$ of the abstract model that we are considering. 
For any bounded $\mathcal{D}^{\ell_{+, \gamma}}$ measurable region $\Lambda$ and any $\vec{s}_{\Lambda^{c}}$ in $L^{\infty}\left(\mathbb{Z}^{d}, S_{Q}\right)$, we define the "dilute", finite volume Gibbs measures on $\mathcal{X}_{\Lambda}^{\hat{p}} \times \mathcal{B}_{\Lambda}^{\hat{p}}$ as,

$$
\mu_{\mathrm{abs}, \Lambda}^{\hat{p}}\left(\vec{\xi}^{\prime}, \underline{\Gamma} \mid \vec{s}_{\Lambda^{c}}\right):=\frac{\mathbf{1}_{\vec{\xi}^{\prime} \in \mathcal{X}^{\hat{p}}}}{Z_{\mathrm{abs}, \beta, \Lambda}^{\hat{p}}\left(\vec{s}_{\Lambda^{c}}\right)} \prod_{\Gamma \in \underline{\Gamma}} W^{\hat{p}}\left(\Gamma, \vec{\xi}^{\prime}\right) e^{-H_{\gamma, \Lambda}\left(\vec{\xi}_{\Lambda}^{\prime} \mid \vec{s}_{\Lambda^{c}}\right)}
$$

The following theorem states the relation between the true model and the abstract ones. Its proof can be easily obtained by induction on the volume, but we omit it here since it is a standard result in Pirogov Sinai theory.

Theorem 4.4. If for any $\hat{p}$ and any $\hat{p}$-contour $\Gamma$ the weights $\hat{W}_{\gamma}^{\hat{p}}(\Gamma ; \vec{\xi})$, defined by (4.7), satisfy

$$
\hat{W}_{\gamma}^{\hat{p}}(\Gamma ; \vec{s})<e^{-\frac{\mathfrak{k}_{\gamma}}{2} N_{\Gamma}}
$$

then

$$
W_{\gamma}^{\hat{p}}(\Gamma ; \vec{s})=w_{\gamma, \beta}^{\hat{p}}(\Gamma ; \vec{s}) \quad ; \quad Z_{\mathrm{abs}, \beta, \Lambda}^{\hat{p}}\left(\vec{s}_{\Lambda^{c}}\right)=Z_{\beta, \Lambda}^{\hat{p}}\left(\vec{s}_{\Lambda^{c}}\right)
$$

Let denote by $P_{\mathrm{abs}, \Lambda, \gamma, \beta}^{\hat{p}}\left(\underline{\vec{\xi}}_{\Lambda^{c}}\right)$ the "finite volume pressure" of the $\hat{p}$-th abstract model:

$$
P_{\mathrm{abs}, \Lambda, \gamma, \beta}^{\hat{p}}\left(\underline{\vec{\xi}}_{\Lambda^{c}}\right):=\frac{1}{\beta|\Lambda|} \ln Z_{\mathrm{abs}, \beta, \Lambda}^{\hat{p}}\left(\underline{\vec{\xi}}_{\Lambda^{c}}\right)
$$

The following theorem characterizes the infinite volume limit of these pressures

Theorem 4.5. Let $\left\{\Lambda_{n}\right\}$ an increasing sequence of sets in $\mathbb{R}^{d}$ of side $2^{n} \ell_{+, \gamma}$. For each $\hat{p}$ in $\{-1,1, \cdots, Q\}$, there exists the limit:

$$
P_{\mathrm{abs}, \gamma, \beta}^{\hat{p}}:=\lim _{n \rightarrow \infty} \frac{1}{\beta\left|\Lambda_{n}\right|} \ln P_{\mathrm{abs}, \Lambda_{n}, \gamma, \beta}^{\hat{p}}\left(\vec{\rho}_{\beta}^{\hat{p}}\right)
$$

which is continuous in $\beta$. Moreover there are constants $c_{b}, \bar{\gamma}$ such that for any $\gamma<\bar{\gamma}$ there is a value of $\beta$, noted $\beta_{c}(\gamma)$, with $\left|\beta_{c}^{\mathrm{mf}}-\beta_{c}(\gamma)\right|<c_{b} \gamma^{1 / 2}$ such that all the pressures are equal

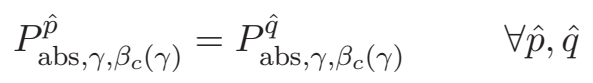

The existence of the limits follows by general arguments for regular interactions and the existence of a (non necessarily unique) value of $\beta$ at which they are equal follows, for $\gamma$ small enough, using a continuity argument and the fact that the mean field pressures are crossing at $\beta=\beta_{c}^{\mathrm{mf}}$. Fixing $\beta=\beta_{c}(\gamma)$ (4.13) holds and the ratios:

$$
\frac{Z_{\mathrm{abs}, \beta, \Lambda}^{\hat{q}}\left(\vec{\rho}^{\hat{q}}\right)}{Z_{\mathrm{abs}, \beta, \Lambda}^{\hat{p}}\left(\vec{\rho}^{\hat{p}}\right)}
$$

converge to 1 in the limit $|\Lambda| \rightarrow \infty$ but the control of the finite volume corrections requires an extra analysis with respect to the standard low temperature case, where the reference configurations are singletons. Here we use a partial cluster expansion to sum 
over the contours using the measure (4.9)) and write its marginal $\mu_{\mathrm{abs}, \Lambda}^{\hat{p}}\left(. \mid \vec{s}_{\Lambda^{c}}\right)$ on $\mathcal{X}_{\Lambda}^{\hat{p}}$. We characterize then the marginals $\mu_{\mathrm{abs}, \Lambda}^{\hat{p}}$ using a generalized Dobrushin argument.

Following Dobrushin, we introduce an interpolation Hamiltonian as follows. Let $u \in$ $[0,1]$ and :

$$
\hat{h}_{u}^{\hat{p}}\left(\vec{\xi}_{\Lambda} \mid \vec{\xi}_{\Lambda^{c}}\right):=u H_{\gamma, \Lambda}\left(\vec{\xi}_{\Lambda} \mid \vec{\xi}_{\Lambda^{c}}\right)+(1-u) \mathfrak{H}_{\gamma, \Lambda}^{\hat{p}}(\vec{\xi})
$$

where $\mathfrak{H}^{\hat{p}}$ are the one body "mean field" Hamiltonians:

$$
\mathfrak{H}_{\gamma, \Lambda}^{\hat{p}}(\vec{\xi}):=\sum_{i \in \Lambda} \sum_{j \neq i} J_{\gamma}(i, j)\left(\left(\vec{\xi}-\vec{\rho}^{\hat{p}}\right) \cdot \vec{\rho}^{\hat{p}}\right)+H_{\gamma, \Lambda}\left(\vec{\rho}^{\hat{p}} \mid \vec{\rho}^{\hat{p}}\right)
$$

We denote by $\mu_{\mathrm{abs}, \Lambda ; u}^{ \pm}\left(\vec{\xi}_{i} \mid \underline{\vec{\xi}}\right)$ the [finite volume] Gibbs measure with hamiltonian $\hat{h}_{u}^{ \pm}(\vec{\xi})$ on $\mathcal{X}_{\Lambda}^{ \pm}$and by $Z_{\mathrm{abs}, \beta, \Lambda ; u}^{ \pm}$the associated partition function. The finite volume pressures of the abstract model can be written in terms of correlations as:

$$
P_{\mathrm{abs}, \gamma, \Lambda}^{\hat{p}}=\frac{1}{\beta|\Lambda|} \ln Z_{\mathrm{abs}, \beta, \Lambda ; 0}^{\hat{p}}-\frac{1}{|\Lambda|} \int_{0}^{1} d u\left\langle\tilde{H}_{\Lambda}^{\hat{p}}\left(\vec{\xi}_{\Lambda}^{\prime} \mid \vec{\rho}^{\hat{p}}\right)-\mathfrak{H}_{\Lambda}^{\hat{p}}\left(\vec{\xi}_{\Lambda}^{\prime}\right)\right\rangle_{\tilde{\mu}_{\mathrm{abs}, \Lambda ; u}^{\hat{p}}}
$$

The estimates for the finite volume corrections to the pressure will follow essentially from the proof of exponentially decay of correlations for the measures $\mu_{\mathrm{abs}, \Lambda ; u}^{\hat{p}}\left(\vec{\xi}_{i} \mid \underline{\xi}\right)$, which is based mainly on the following two results:

Theorem 4.6. For any $i \in \mathbb{Z}^{d}$ there is a measurable set $G_{i}^{\hat{p}} \sqsubset \mathcal{X}^{\hat{p}}$ depending only on $\left\{\underline{\vec{\xi}}_{j}, j \in C_{i}^{\ell-, \gamma} \backslash i\right\}$, such that there exists $b(i, j)$ with the following properties:

$$
\begin{aligned}
& b(i, j) \geq 0 ; b(i, i)=0 \\
& \sup _{i \in \mathbb{Z}^{d}} \sum_{j \in C_{i}^{\ell-, \gamma}} b(i, j)<\delta<1 \\
& \mathcal{R}\left(\mu_{\mathrm{abs}, i ; u}^{\hat{p}}\left(\vec{\xi}_{i} \mid \underline{\vec{\xi}}\right), \mu_{\mathrm{abs}, i ; u}^{\hat{p}}\left(\vec{\xi}_{i} \mid \underline{\vec{\xi}}\right)\right) \leq \sum_{j} b(i, j) \operatorname{dist}\left(\underline{\vec{\xi}}_{j}, \underline{\xi}_{j}\right) \quad \text { for any } \underline{\vec{\xi}}, \underline{\vec{\xi}} \in G_{i} \text { and any } u \in(0,1)
\end{aligned}
$$

where $\mathcal{R}\left(\mu_{\mathrm{abs}, i ; u}^{\hat{p}}\left(\vec{\xi}_{i} \mid \underline{\xi}\right), \mu_{\mathrm{abs}, i ; u}^{\hat{p}}\left(\vec{\xi}_{i} \mid \underline{\xi}\right)\right)$ is the Vaserstein distance associated to the metric on the configuration space defined by:

$$
\operatorname{dist}\left(\vec{\xi}_{i}, \vec{\xi}_{i}^{\prime}\right):=\frac{1}{2} \sum_{i}\left|\xi_{i}(i)-\xi_{i}^{\prime}(i)\right|
$$

We will prove that the theorem holds with $G_{i}^{\hat{p}}$ defined as :

$$
G_{i}^{\hat{p}}:=\left\{\vec{\xi} \in \mathcal{X}^{(\hat{p})}: \vec{\xi}^{(i, q)} \in \mathcal{X}^{(\hat{p})} \forall q\right\}
$$

where we have denoted

$$
\vec{\xi}_{j}^{(i, q)}= \begin{cases}\vec{\xi}_{j} & j \neq i, \\ \vec{u}_{q} & j=i .\end{cases}
$$

$G_{i}^{\hat{p}}$ is then the set of configurations $\vec{\xi}$ which belong to $\mathcal{X}^{(\hat{p})}$ independently of the value of $\vec{\xi}_{i}$ and it is measurable on $\vec{\xi}_{i^{c}}$. 
Notice that when $\underline{\vec{\xi}}, \underline{\vec{\xi}}$ are not in $G_{i}^{\hat{p}}$, the probability measures for $\vec{\xi}_{i}$ have support on a strict subset of $\Omega$ and the statement of the theorem 4.6 would not hold in general.

The other result that allows to prove exponentially decay of the correlations is a bound on the probability of the "bad set" of configurations, $G_{i}^{\hat{p}^{c}}$ :

$$
\sup _{\overrightarrow{\xi^{*}} \in \mathcal{X}^{\hat{p}}} \mu_{\mathrm{abs}, D, u}^{(\hat{p})}\left(G_{i}^{\hat{p}^{c}} \mid \vec{\xi}^{*}\right) \leq e^{-c \gamma^{2 a} \ell_{-, \gamma}^{d}}
$$

$c$ a positive constant and $D=C_{i}^{\ell_{-, \gamma}}$.

Together with the exponential decay of the correlations, we need also to have a control on the contribution of the parts close to the boundary. This control follows from the next theorem that, in words, states that well inside a "correct region" (i.e. $\Theta=\hat{q}, \hat{q} \neq 0$ ), the typical configurations becomes "very close" (i.e. on the small scale $\ell_{0}$ ) to the corresponding mean field value.

Theorem 4.7. Let $A$ a finite subset of $\mathbb{Z}^{d}, \hat{A}:=A \sqcup \delta_{\text {out }}^{\ell_{+} \gamma}[A], \hat{h}_{u}\left(\vec{\xi}_{\hat{A}} \mid s_{\hat{A}^{c}}\right)$ as in (17.3) and $\hat{\mu}_{\text {abs; } \hat{A}, u}^{\hat{p}}$ is the measure on $\mathcal{X}_{\hat{A}}^{\hat{p}}$ associated $\hat{h}_{u}^{\hat{p}}\left(\vec{\xi}_{\hat{A}^{\prime}} \mid \vec{\xi}_{\hat{A}^{c}}\right)$, then uniformly in $u$ :

$$
\hat{\mu}_{\mathrm{abs} ; \hat{A}, u}^{\hat{p}}\left(\mathbf{1}_{\left|S_{A}^{\hat{p}}(\vec{\xi})\right| \geq \gamma^{1 / 8}|A|}\right) \leq e^{-c \gamma^{3 / 8}|A|}
$$

where:

$$
S_{A}^{\hat{p}}(\vec{\xi}):=\left\{i \in A \sqcup \delta_{\text {out }}^{\gamma^{-1}}[A]:\left\|\vec{\xi}^{\left(\ell_{0}\right)}(i)-\vec{\rho}^{\hat{p}}\right\|_{\star} \geq \gamma^{1 / 8}\right\}
$$

Now collecting all these results, we get an estimate for the ratio of partition functions of two abstract models, We then prove the following theorem:

Theorem 4.8. There is $\bar{\gamma}$, and a constant $\kappa_{1}$ such that for any $\gamma<\bar{\gamma}$, there is a value of $\beta, \beta_{c}(\gamma)$ :

$$
\left|\ln \left(\frac{Z_{\mathrm{abs}, \beta_{c}(\gamma), \Lambda}^{(+)}\left(\vec{\rho}^{(+)}\right) e^{R_{\gamma, \Lambda}^{\mathrm{mf}, \hat{q}}}}{Z_{\mathrm{abs}, \beta_{c}(\gamma), \Lambda}^{(-)}\left(\vec{\rho}^{(-)}\right) e^{R_{\gamma, \Lambda}^{\mathrm{mf}, \hat{p}}}}\right)\right| \leq \kappa_{1} \gamma^{1 / 8}|\Gamma|
$$

where $R_{\gamma, \Lambda}^{\mathrm{mf}, \hat{p}}$ are the mean-field finite volume corrections to the pressures,

$$
R_{\gamma, \Lambda}^{\mathrm{mf}, \hat{p}}:=\frac{\beta}{2} \sum_{\substack{x \in \Lambda \\ y \in \Lambda^{c}}} J_{\gamma}(x, y)\left(\hat{\rho}^{\hat{p}} \cdot \vec{\rho}^{\hat{p}}\right)
$$

Deriving a factorization theorem similar to Theorem 4.2 for the abstract contours models lead then to a bound for the abstract weights as in Theorem 4.3 Hence, using Theorem 4.4 we can identify abstract and true weights at temperature $\beta=\beta_{c}(\gamma)$ and in turn prove Theorem 4.3 whiich lead to our result.

In the next sections we will proceed by proving all results presented here, but in a different order. Precisely we will postpone the proof of the Theorem 4.2 and start the analysis of the abstract models, their pressures and the uniqueness of the associated measures. 
Finally we will prove the large deviation estimate needed in 4.3 As a preliminary step we introduce the mean field functional whose minimizers define the unperturbed states above which the Pirogov-Sinai analysis is developed, and discuss its properties.

\section{Coarse graining and mean field functional}

Let $\ell$ a large positive integer and $\mathcal{D}^{\ell}$ a partition of $\mathbb{R}^{d}$ in cubes of size $\ell$. For all $x$ in $\mathbb{R}^{d}$, we denote by $C_{x}^{\ell}$ the cube of $\mathcal{D}^{\ell}$ containing $x$. We define a coarse-grained configuration on $\mathcal{D}^{\ell}$ as follows: for each configuration $\vec{\xi} \in \Omega$ and any $x \in \mathbb{R}^{d}$, the coarse-grained configuration (at scale $\ell$ ) is the $\mathrm{Q}$-dimensional vector,

$$
\vec{\xi}^{\ell}(x)=\ell^{-d} \sum_{i \in C_{x}^{\ell}} \vec{\xi}(i)
$$

where we make use of our notational conventions. The $k^{t h}$ component $\xi_{k}^{\ell}(x)$ is the empirical density of color $a_{k}$ in $C_{x}^{\ell}$. Due to the underlying discretization, $\vec{\xi}^{\ell}(x)$ takes values in the finite set $M_{\ell^{d}}^{Q}$

$$
M_{\ell^{d}}^{Q}=\left\{\left(r_{1}, \cdots, r_{Q}\right) ;\left(\ell^{d} r_{k}\right) \in \mathbb{N}, \sum_{k=1}^{Q} r_{k}=1\right\}
$$

Let $\Lambda$ a $\mathcal{D}^{\ell}$-measurable subset of $\mathbb{R}^{d}$. The set of coarse-grained configurations in $\Lambda$ is denoted by $\Omega_{\Lambda}^{\ell}$ and corresponds to the set of $\mathcal{D}^{\ell}$-measurable functions on $\Lambda$ with values in $M_{\ell^{d}}^{Q}$. We extend the discrete set $M_{\ell^{d}}^{Q}$ to the simplex $S_{Q}$ in $\mathbb{R}^{Q}$ defined in (2.5). Thus all coarse-grained configurations in $\Omega_{\Lambda}^{\ell}$ are also elements of $L^{\infty}\left(\Lambda, S_{Q}\right)$. Conversely we will approximate any function in $L^{\infty}\left(\Lambda, S_{Q}\right)$ by a coarse grained configuration. For any $\vec{\rho} \in L^{\infty}\left(\Lambda, S_{Q}\right)$, we will denote by $\vec{\rho}^{\ell}$ its $\mathcal{D}^{\ell}$-measurable approximation

$$
\vec{\rho}^{\ell}(x)=\ell^{-d} \int_{C_{x}^{\ell}} \vec{\rho}(y) d y
$$

for all $x$ in $\Lambda$, and by $[\vec{\rho}]^{\ell}$ the only function in $\Omega_{\Lambda}^{\ell}$ such that

$$
-\frac{1}{2 \ell^{d}}<[\vec{\rho}]_{k}^{\ell}(x)-\rho_{k}^{\ell}(x) \leq \frac{1}{2 \ell^{d}}
$$

for all $k$ and all $x$ in $\Lambda$.

For $\Lambda$ a finite $\mathcal{D}^{\ell}$-measurable region in $\mathbb{R}^{d}$, we define the mean field free energy functional on $L^{\infty}\left(\Lambda, S_{Q}\right)$ by,

$$
F_{\gamma, \beta, \Lambda}\left(\vec{\rho}_{\Lambda} \mid \vec{\rho}_{\Lambda^{c}}\right)=V_{\gamma, \Lambda}\left(\vec{\rho}_{\Lambda} \mid \vec{\rho}_{\Lambda^{c}}\right)-\frac{1}{\beta} I\left(\vec{\rho}_{\Lambda}\right)
$$

where $\vec{\rho}_{\Lambda^{c}} \in L^{\infty}\left(\Lambda^{c}, S_{Q}\right)$ defines the boundary conditions. The two functionals $V_{\gamma, \Lambda}\left(\vec{\rho}_{\Lambda} \mid \vec{\rho}_{\Lambda^{c}}\right)$ and $I\left(\vec{\rho}_{\Lambda}\right)$ are respectively the energy and the entropy of configuration $\vec{\rho}_{\Lambda}$,

$V_{\gamma, \Lambda}\left(\vec{\rho}_{\Lambda} \mid \vec{\rho}_{\Lambda^{c}}\right)=-\frac{1}{2} \int_{\Lambda} \int_{\Lambda} J_{\gamma}(x, y)\left(\vec{\rho}_{\Lambda}(x) \cdot \vec{\rho}_{\Lambda}(y)\right) d x d y-\int_{\Lambda} \int_{\Lambda^{c}} J_{\gamma}(x, y)\left(\vec{\rho}_{\Lambda}(x) \cdot \vec{\rho}_{\Lambda^{c}}(y)\right) d x d y$ 


$$
I\left(\vec{\rho}_{\Lambda}\right)=-\int_{\Lambda} \sum_{q=1}^{Q} \rho_{\Lambda, q}(x) \ln \left(\rho_{\Lambda, q}(x)\right) d x=:-\int_{\Lambda} \vec{\rho}_{\Lambda}(x) \cdot \ln \vec{\rho}_{\Lambda}(x) d x
$$

For all $\mathcal{A} \sqsubset \Omega_{\Lambda}$ we define the constrained partition function $Z_{\gamma, \beta, \Lambda}\left(\mathcal{A} \mid \vec{\xi}_{\Lambda^{c}}\right)$

$$
Z_{\gamma, \beta, \Lambda}\left(\mathcal{A} \mid \vec{\xi}_{\Lambda^{c}}\right)=\sum_{\vec{\xi}_{\Lambda} \in \mathcal{A}} e^{-\beta H_{\gamma, \Lambda}\left(\vec{\xi}_{\Lambda} \mid \vec{\xi}_{\Lambda^{c}}\right)}
$$

We now state a theorem relating constrained partition functions and mean field free energy:

Theorem 5.1. There exists a constant $c>0$ such that for all $\gamma>0, \ell \in\left(1, \gamma^{-1}\right)$ and all bounded $\mathcal{D}^{\ell}$-measurable region $\Lambda$ of $\mathbb{R}^{d}$, the following inequalities hold:

For all subsets $\mathcal{A}$ of $\Omega_{\Lambda}^{\ell}$,

$$
\ln Z_{\gamma, \beta, \Lambda}\left(\left\{\vec{\xi}_{\Lambda}^{\ell} \in \mathcal{A}\right\} \mid \vec{\xi}_{\Lambda^{c}}\right)+\beta \inf _{\vec{\rho}_{\Lambda} \in \mathcal{A}} F_{\gamma, \beta, \Lambda}\left(\vec{\rho}_{\Lambda} \mid \vec{\xi}_{\Lambda^{c}}^{\ell}\right) \leq \beta c \epsilon(\gamma, \ell)|\Lambda|
$$

and for all $\vec{\rho}_{\Lambda} \in L^{\infty}\left(\Lambda, S_{Q}\right)$,

$$
\ln Z_{\gamma, \beta, \Lambda}\left(\left\{\vec{\xi}_{\Lambda}: \vec{\xi}_{\Lambda}^{\ell}=\left[\vec{\rho}_{\Lambda}\right]^{\ell}\right\} \mid \vec{\xi}_{\Lambda^{c}}\right)+\beta F_{\gamma, \beta, \Lambda}\left(\vec{\rho}_{\Lambda} \mid \vec{\xi}_{\Lambda^{c}}^{\ell}\right) \geq-\beta c \epsilon(\gamma, \ell)|\Lambda|
$$

where

$$
\epsilon(\gamma, \ell)=\gamma \ell+\frac{\ln \ell}{\ell^{d}}
$$

Proof. We first estimate the difference between the energy $H_{\gamma, \Lambda}\left(\vec{\xi}_{\Lambda} \mid \vec{\xi}_{\Lambda^{c}}\right)$ and its coarsegrained approximation $V_{\gamma, \Lambda}\left(\vec{\xi}_{\Lambda}^{\ell} \mid \vec{\xi}_{\Lambda^{c}}^{\ell}\right)$. Given two cubes $C_{1}$ and $C_{2}$ of the partition $\mathcal{D}^{\ell}$, for any two points $i \in C_{1}$ and $j \in C_{2}$, we have

$$
\left|J_{\gamma}(i, j)-\frac{1}{\ell^{2 d}} \int_{C_{1} \times C_{2}} J_{\gamma}(x, y) d x d y\right| \leq 2 \sqrt{d}\|\nabla \mathcal{J}\|_{\infty} \gamma^{d+1} \ell \mathbf{1}_{d(C 1, C 2) \leq \gamma^{-1}}
$$

where $d(C 1, C 2)=\inf _{x \in C_{1}, y \in C_{2}}|x-y|$.

Hence

$$
\begin{aligned}
\left|H_{\gamma, \Lambda}\left(\vec{\xi}_{\Lambda} \mid \vec{\xi}_{\Lambda^{c}}\right)-V_{\gamma, \Lambda}\left(\vec{\xi}_{\Lambda}^{\ell} \mid \vec{\xi}_{\Lambda^{c}}^{\ell}\right)\right| & \leq \sum_{\substack{C_{1} \in \mathcal{D}_{\Lambda}^{\ell} \\
C_{2} \in \mathcal{D}^{\ell}}}\left|\sum_{\substack{i \in C_{1} \cap \mathbb{Z}^{d} \\
j \in C_{2} \cap \mathbb{Z}^{d}}} J_{\gamma}(i, j) \vec{\xi}_{\Lambda}(i) \cdot \vec{\xi}_{\Lambda}(j)-\int_{C_{1} \times C_{2}} J_{\gamma}(x, y) \vec{\xi}_{\Lambda}^{\ell}(x) \cdot \vec{\xi}_{\Lambda}^{\ell}(y)\right| \\
\leq & \sum_{\substack{C_{1} \in \mathcal{D}_{\Lambda}^{\ell} \\
C_{2} \in \mathcal{D}^{\ell}}}\left|\sum_{\substack{i \in C_{1} \cap \mathbb{Z}^{d} \\
j \in C_{2} \cap \mathbb{Z}^{d}}}\left(J_{\gamma}(i, j)-\frac{1}{\ell^{2 d}} \int_{C_{1} \times C_{2}} J_{\gamma}(x, y) d x d y\right) \vec{\xi}_{\Lambda}(i) \cdot \vec{\xi}_{\Lambda}(j)\right| \\
\leq & 2 \sqrt{d}\|\nabla \mathcal{J}\|_{\infty} \gamma^{d+1} \ell \sum_{C_{1}, C_{2}}\left|C_{1}\right|\left|C_{2}\right| \mathbf{1}_{d\left(C_{1}, C_{2}\right) \leq \gamma^{-1}} \\
\leq & c_{d} \gamma \ell|\Lambda|
\end{aligned}
$$

where $c_{d}$ is a constant independent on $\gamma, \ell \leq \gamma^{-1}$ and $\Lambda$

$$
c_{d}=23^{d} \sqrt{d}\|\nabla \mathcal{J}\|_{\infty}
$$


Thus for any profile $\vec{\rho}_{\Lambda}$ in $\Omega_{\Lambda}^{\ell}$, we have

$$
\left|\ln \frac{Z_{\gamma, \beta, \Lambda}\left(\left\{\vec{\xi}_{\Lambda}: \vec{\xi}_{\Lambda}^{\ell}=\vec{\rho}_{\Lambda}\right\} \mid \vec{\xi}_{\Lambda^{c}}\right)}{\left|\left\{\vec{\xi}_{\Lambda}: \vec{\xi}_{\Lambda}^{\ell}=\vec{\rho}_{\Lambda}\right\}\right| e^{-\beta V_{\gamma, \Lambda}\left(\vec{\rho}_{\Lambda} \mid \vec{\xi}_{\Lambda^{c}}^{\ell}\right)}}\right| \leq c_{d} \gamma \ell|\Lambda|
$$

The cardinality of $\left\{\vec{\xi}_{\Lambda}: \vec{\xi}_{\Lambda}^{\ell}=\vec{\rho}_{\Lambda}\right\}$ can be related to the entropy of $\vec{\rho}_{\Lambda}$. The error bounds for the Stirling formula

$$
\frac{1}{12 N+1} \leq \frac{\ln (N !)}{N(\ln (N)-1)+\ln (\sqrt{2 \pi N})} \leq \frac{1}{12 N}
$$

lead to the following estimate

$$
|\ln |\left\{\vec{\xi}_{\Lambda}: \vec{\xi}_{\Lambda}^{\ell}=\vec{\rho}_{\Lambda}\right\}\left|-I\left(\vec{\rho}_{\Lambda}\right)\right| \leq d Q|\Lambda| \frac{\log \ell}{\ell^{d}}
$$

which implies

$$
\left|\ln Z_{\gamma, \beta, \Lambda}\left(\left\{\vec{\xi}_{\Lambda}: \vec{\xi}_{\Lambda}^{\ell}=\vec{\rho}_{\Lambda}\right\} \mid \vec{\xi}_{\Lambda^{c}}\right)+\beta F_{\gamma, \beta, \Lambda}\left(\vec{\rho}_{\Lambda} \mid \vec{\xi}_{\Lambda^{c}}^{\ell}\right)\right| \leq \beta c_{d} \gamma \ell|\Lambda|+Q d|\Lambda| \frac{\log \ell}{\ell^{d}}
$$

Now an easy upper bound for the cardinality of $\Omega_{\Lambda}^{\ell}$ gives for all $\mathcal{A} \sqsubset \Omega_{\Lambda}^{\ell}$

$$
\ln |\mathcal{A}| \leq \ln \left|\Omega_{\Lambda}^{\ell}\right| \leq Q d \frac{|\Lambda|}{\ell^{d}} \log \ell
$$

where $|\mathcal{A}|$ denotes the cardinality of the set $\mathcal{A}$. Combining these two last inequalities gives the first part of Theorem 5.1 with $c=\max \left(c_{d}, \frac{2 Q d}{\beta}\right)$. On the other side, for all $\vec{\rho} \in L^{\infty}\left(\Lambda, S_{Q}\right)$, one has

$$
\log Z_{\gamma, \beta, \Lambda}\left(\vec{\xi}_{\Lambda}^{\ell}=\left[\vec{\rho}_{\Lambda}\right]^{\ell} \mid \vec{\xi}_{\Lambda^{c}}\right) \geq-\beta F_{\gamma, \beta, \Lambda}\left([\vec{\rho}]^{\ell} \mid \vec{\xi}_{\Lambda^{c}}^{\ell}\right)-c \epsilon(\gamma, \ell)|\Lambda|
$$

Now using

$$
\left|V_{\gamma, \Lambda}\left(\vec{\rho}_{\Lambda} \mid \vec{\xi}_{\Lambda^{c}}^{\ell}\right)-V_{\gamma, \Lambda}\left(\vec{\rho}_{\Lambda}^{\ell} \mid \vec{\xi}_{\Lambda^{c}}^{\ell}\right)\right| \leq c_{d} \gamma|\Lambda|
$$

and the concavity of the entropy, one gets

$$
F_{\gamma, \beta, \Lambda}\left(\vec{\rho}_{\Lambda} \mid \vec{\xi}_{\Lambda^{c}}^{\ell}\right) \geq F_{\gamma, \beta, \Lambda}\left(\vec{\rho}_{\Lambda}^{\ell} \mid \vec{\xi}_{\Lambda^{c}}^{\ell}\right)-c_{d} \gamma \ell|\Lambda|
$$

Furthermore, approximating $\vec{\rho}_{\Lambda}^{\ell}$ by $\left[\vec{\rho}_{\Lambda}\right]^{\ell}$ gives

$$
F_{\gamma, \beta, \Lambda}\left(\vec{\rho}_{\Lambda}^{\ell} \mid \vec{\xi}_{\Lambda^{c}}^{\ell}\right) \geq F_{\gamma, \beta, \Lambda}\left(\left[\vec{\rho}_{\Lambda}\right]^{\ell} \mid \vec{\xi}_{\Lambda^{c}}^{\ell}\right)-d Q \frac{|\Lambda|}{\ell^{d}}
$$

we thus get:

$$
\log Z_{\gamma, \beta, \Lambda}\left(\vec{\xi}_{\Lambda}^{\ell}=[\vec{\rho}]^{\ell} \mid \vec{\xi}_{\Lambda^{c}}\right) \geq-\beta F_{\gamma, \beta, \Lambda}\left(\vec{\rho}_{\Lambda} \mid \vec{\xi}_{\Lambda^{c}}^{\ell}\right)-\beta c \epsilon(\gamma, \ell)|\Lambda|
$$

which gives the second part of the theorem with the same constant as before.

We will use theorem 5.1 mostly in the following weaker form:

Corollary 5.2. There exists a constant $c>0$ such that for all $\gamma>0, \ell$ an integer in $\left(1, \gamma^{-1}\right)$ and all bounded $\mathcal{D}^{\ell}$-measurable regions $\Lambda$ in $\mathbb{R}^{d}$,

$$
\left|\log Z_{\gamma, \beta, \Lambda}\left(\vec{\xi}_{\Lambda^{c}}\right)+\beta \inf _{\vec{\rho}_{\Lambda}} F_{\gamma, \beta, \Lambda}\left(\vec{\rho}_{\Lambda} \mid \vec{\xi}_{\Lambda^{c}}^{\ell}\right)\right| \leq \beta c \epsilon(\gamma, \ell)|\Lambda|
$$

Theorem 5.1 leads also to the Lebowitz-Penrose limit for the Potts model 
Theorem 5.3. [Lebowitz-Penrose] There exists the limit

$$
\lim _{\gamma \rightarrow 0} \lim _{\Lambda \rightarrow \mathbb{R}^{d}} \frac{\log Z_{\gamma, \beta, \Lambda}}{|\Lambda|}=P_{\beta}^{\mathrm{mf}}
$$

where $P_{\beta}^{\mathrm{mf}}$ is the mean field pressure.

Proof. The free energy functional on $L^{\infty}\left(\Lambda, S_{Q}\right)$ with boundary conditions $\vec{\rho}_{\Lambda^{c}}$ can be rewritten as

$$
F_{\gamma, \beta, \Lambda}\left(\vec{\rho}_{\Lambda} \mid \vec{\rho}_{\Lambda^{c}}\right)=\mathcal{F}_{\gamma, \beta, \Lambda}\left(\vec{\rho}_{\Lambda} \mid \vec{\rho}_{\Lambda^{c}}\right)-\frac{1}{2} \int_{\Lambda} \int_{\Lambda^{c}} J_{\gamma}(x, y)\left|\vec{\rho}_{\Lambda^{c}}(y)\right|^{2} d x d y
$$

where

$$
\begin{aligned}
\mathcal{F}_{\gamma, \beta, \Lambda}\left(\vec{\rho}_{\Lambda} \mid \vec{\rho}_{\Lambda^{c}}\right)= & \int_{\Lambda} \phi^{\operatorname{mf}}\left(\vec{\rho}_{\Lambda}(x)\right) d x+\frac{1}{4} \int_{\Lambda} \int_{\Lambda} J_{\gamma}(x, y)\left|\vec{\rho}_{\Lambda}(x)-\vec{\rho}_{\Lambda}(y)\right|^{2} d x d y \\
& +\frac{1}{2} \int_{\Lambda} \int_{\Lambda^{c}} J_{\gamma}(x, y)\left|\vec{\rho}_{\Lambda}(x)-\vec{\rho}_{\Lambda^{c}}(y)\right|^{2} d x d y
\end{aligned}
$$

with $\phi_{\beta}^{\mathrm{mf}}(\vec{v})$ the mean field free energy density on $S_{Q}:=\left\{\vec{v} \in \mathbb{R}_{+}^{Q}, \sum_{q} \rho_{q}=1\right\}$

$$
\phi_{\beta}^{\operatorname{mf}}(\vec{v}):=\frac{1}{2}(\vec{v} \cdot \vec{v})-\frac{1}{\beta} \vec{v} \cdot \ln (\vec{v})
$$

(see (A.2)).

We have clearly

$$
\inf _{\vec{\rho}_{\Lambda} \in L^{\infty}\left(\Lambda, S_{Q}\right)} \mathcal{F}_{\gamma, \beta, \Lambda}\left(\vec{\rho}_{\Lambda} \mid \vec{\rho}_{\Lambda^{c}}\right) \geq \inf _{\vec{\rho}_{\Lambda} \in L^{\infty}\left(\Lambda, S_{Q}\right)} \int_{\Lambda} \phi_{\beta}^{\mathrm{mf}}\left(\vec{\rho}_{\Lambda}(x)\right) d x
$$

which gives a lower bound for the free energy as

$$
\inf _{\vec{\rho}_{\Lambda} \in L^{\infty}\left(\Lambda, S_{Q}\right)} F_{\gamma, \beta, \Lambda}\left(\vec{\rho}_{\Lambda} \mid \vec{\rho}_{\Lambda^{c}}\right) \geq-P_{\beta}^{\mathrm{mf}}|\Lambda|-c \gamma^{-1}|\partial \Lambda|
$$

with

$$
P_{\beta}^{\operatorname{mf}}=-\inf _{\vec{v} \in S_{Q}} \phi_{\beta}^{\mathrm{mf}}(\vec{v})
$$

From 5.1 with $\ell=\gamma^{-\frac{1}{2}}$, one gets

$$
\frac{\log Z_{\gamma, \beta, \Lambda}}{\beta|\Lambda|} \leq P_{\beta}^{\mathrm{mf}}+c \epsilon\left(\gamma, \gamma^{-1 / 2}\right)+\gamma^{-1} \frac{|\partial \Lambda|}{|\Lambda|}
$$

and hence

$$
\limsup _{\gamma \rightarrow 0} \limsup _{\Lambda \nearrow \mathbb{R}^{d}} \frac{\log Z_{\gamma, \beta, \Lambda}}{\beta|\Lambda|} \leq P_{\beta}^{\mathrm{mf}}
$$

where the limit limsup $\operatorname{su}_{\Lambda} \mathbb{R}^{d}$ is taken on a sequence of Van Hove subsets of $\mathbb{R}^{d}$. On the other side, writing (5.32) for $\vec{\rho}_{\Lambda}=\vec{\rho}^{\hat{p}} \mathbf{1}_{\Lambda}$, where $\vec{\rho}^{\hat{p}}$ is an absolute minimizer of $\phi_{\beta}^{\mathrm{mf}}$, we get

$$
F_{\gamma, \beta, \Lambda}\left(\vec{\rho}^{*} \mathbf{1}_{\Lambda} \mid \vec{\rho}_{\Lambda^{c}}\right) \leq \mathcal{F}_{\gamma, \beta, \Lambda}\left(\vec{\rho}^{*} \mathbf{1}_{\Lambda} \mid \vec{\rho}_{\Lambda^{c}}\right) \leq-P_{\beta}^{\mathrm{mf}}|\Lambda|+c \gamma^{-1}|\partial \Lambda|
$$

From the second inequality in 5.1 one gets

$$
\frac{\log Z_{\gamma, \beta, \Lambda}}{\beta|\Lambda|} \geq P_{\beta}^{\mathrm{mf}}-c \epsilon\left(\gamma, \gamma^{-1 / 2}\right)+\gamma^{-1} \frac{|\partial \Lambda|}{|\Lambda|}
$$


and finally

$$
\liminf _{\gamma \rightarrow 0} \liminf _{\Lambda \nearrow \mathbb{R}^{d}} \frac{\log Z_{\gamma, \beta, \Lambda}}{\beta|\Lambda|} \geq P_{\beta}^{\mathrm{mf}}
$$

\section{Analysis of the abstract contour models: equality of the pressures}

In this section and in the following, we will analyse the abstract contours models. A preliminary technical step is a partial cluster expansion of the contours contribution to the partition function against fixed configurations.

\section{A partial cluster expansion}

From a technical point of view the we will take advantage of dealing with truncated weights (4.7) making a partial cluster expansion of the contours, against fixed configurations

getting a partition function with an extra interaction, an "effettive hamiltonian " $\mathcal{H}_{\gamma, \Lambda}^{\hat{p}}$, with infinite range but exponentially decaying.

$$
\sum_{\underline{\Gamma} \in \mathcal{B}_{\Lambda}^{\hat{p}}} \hat{W}_{\gamma}^{\hat{p}}(\underline{\Gamma} ; \vec{\xi})=e^{-\beta \mathcal{H}_{\gamma, \Lambda}^{\hat{p}}\left(\vec{\xi}_{\Lambda}\right)}
$$

A precise statement is given in the following theorem:

Theorem 6.1. If the weights $W^{\hat{p}}(\Gamma, \vec{\xi})$ satisfy the Peierls bounds with a constant $\mathfrak{K}_{\gamma}$ large enough then, for any bounded, $\mathcal{D}^{\ell_{+, \gamma}-m e a s u r a b l e ~ r e g i o n ~} \Lambda$ and any $\vec{\xi} \in \mathcal{X}^{\hat{p}}$,

$$
\begin{gathered}
Z_{\mathrm{abs}, \beta, \Lambda}^{\hat{p}}\left(\underline{\vec{\xi}}_{\Lambda^{c}}\right)=\sum_{\underline{\Gamma} \in \mathcal{B}_{\Lambda}^{\hat{p}}} \sum_{\vec{\xi}^{\prime} \in \mathcal{X}_{\Lambda}^{\hat{p}}} \hat{W}_{\gamma}^{\hat{p}}\left(\underline{\Gamma}, ; \vec{\xi}^{\prime}\right) e^{-\beta H_{\gamma, \Lambda}\left(\vec{\xi}_{\Lambda}^{\prime} \mid \vec{\xi}_{\Lambda^{c}}\right)}=\sum_{\vec{\xi}^{\prime} \in \mathcal{X}_{\Lambda}^{\hat{p}}} e^{-\beta \tilde{H}_{\gamma, \Lambda}^{\hat{p}}\left(\vec{\xi}_{\Lambda}^{\prime} \mid \vec{\xi}_{\Lambda^{c}}\right)} \\
\tilde{H}_{\gamma, \Lambda}^{\hat{p}}\left(\vec{\xi}_{\Lambda}^{\prime} \mid \vec{\xi}_{\Lambda^{c}}\right):=H_{\gamma, \Lambda}\left(\vec{\xi}_{\Lambda}^{\prime} \mid \vec{\xi}_{\Lambda^{c}}\right)+\mathcal{H}_{\gamma, \Lambda}^{\hat{p}}\left(\vec{\xi}_{\Lambda}^{\prime}\right) \\
\mathcal{H}_{\gamma, \Lambda}^{\hat{p}}(\vec{\xi})=\sum_{\Delta \sqsubseteq \Lambda} U_{\Delta}^{\hat{p}}(\vec{\xi})
\end{gathered}
$$


where the potentials $U_{\Delta}^{\hat{p}}\left(\vec{\xi}_{\Delta}\right)$ are defined by (F.11) and satisfy:

$$
\begin{aligned}
& U_{\Delta}^{\hat{p}}=0 \quad \text { if } \Delta \text { is not connected } \\
& \beta \sum_{\Delta \ni x}\left|U_{\Delta}^{\hat{p}}\left(\vec{\xi}_{\Delta}\right)\right| \leq e^{-\frac{\mathfrak{k}_{\gamma}}{2}} \\
& \beta \sum_{\Delta \ni x, \Delta \sqcap A \neq \emptyset} \leq 3^{d} e^{-\frac{\mathfrak{k}_{\gamma}}{2} N_{x, A}} \quad \forall A \sqsubset \mathbb{R}^{d}
\end{aligned}
$$

where $N_{x, A}$ is the minimal number of $\mathcal{D}^{\ell_{+, \gamma}}$-cubes needed to cover the distance between

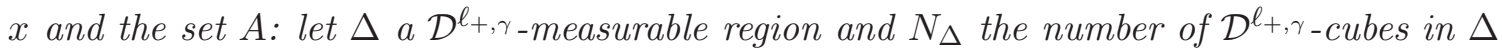

$$
\begin{array}{rl}
N_{x, A} & :=\min _{\Delta}\left\{N_{\Delta}: \Delta \sqcap\left(C_{x} \sqcup \delta_{\text {out }}^{\ell_{+}, \gamma}\left[C_{x}\right]\right) \neq \emptyset, \Delta \sqcap\left(A \sqcup \delta_{\text {out }}^{\ell_{+}, \gamma}[A]\right) \neq \emptyset ; \exists \Gamma: \operatorname{sp}_{+}(\Gamma)=\Delta\right\} \\
C_{x} & a \mathcal{D}^{\ell_{+}, \gamma} \text {-cube containing the point } x .
\end{array}
$$

The proof of the Theorem 6.1 is standard and is given in appendix [F We notice that $N_{x, A}$ in (6.7)-(6.8) is s.t. there is a constant $k$, s.t.:

$$
N_{x, A} \geq \max \left\{3^{d}, k \frac{\operatorname{dist}(x, A)}{\ell_{+, \gamma}}\right\}
$$

The Gibbs measures relative to $\tilde{H}_{\gamma, \Lambda}^{\hat{p}}\left(\vec{\xi}_{\Lambda}^{\prime} \mid \vec{\xi}_{\Lambda^{c}}\right)$ :

$$
\tilde{\mu}_{\mathrm{abs}, \Lambda}^{\hat{p}}\left(\vec{\xi}_{\Lambda} \mid \underline{\xi}_{\Lambda^{c}}\right):=\frac{e^{-\beta \tilde{H}_{\gamma, \Lambda}^{\hat{p}}\left(\vec{\xi}_{\Lambda} \mid \vec{\xi}_{\Lambda^{c}}\right)}}{Z_{\mathrm{abs}, \beta, \Lambda}^{\hat{p}}\left(\underline{\vec{\xi}}_{\Lambda^{c}}\right)}
$$

are the marginals on $\mathcal{X}^{\hat{p}}$ of the measures $\mu_{\mathrm{abs}, \Lambda}^{\hat{p}}\left(\vec{\xi}, \underline{\Gamma} \underline{\xi}_{\Lambda^{c}}\right)$.

In the remainig part of this section and in the next one, we will only consider the two abstract models for $\hat{p}= \pm 1$ since all the others can be deduced from $\hat{p}=1$ by symmetry, and write a superscript \pm instead of $\hat{p}$ to distinguish them. We denote by

$$
P_{\mathrm{abs}, \Lambda, \gamma, \beta}^{ \pm}\left(\underline{\vec{\xi}}_{\Lambda^{c}}\right):=\frac{1}{\beta|\Lambda|} \ln Z_{\mathrm{abs}, \beta, \Lambda}^{ \pm}\left(\underline{\vec{\xi}}_{\Lambda^{c}}\right)
$$

the "finite volume pressures" of the two abstract models.

The proof of Theorem 4.8 requires the proof of the following theorem to control the bulk contribution to the ratio in (4.24):

Theorem 6.2. Let $\left\{\Lambda_{n}\right\}$ a sequence of sets in $\mathbb{R}^{d}$ of side $2^{n} \ell_{+, \gamma}$. There exist the two limits:

$$
P_{\text {abs }, \gamma, \beta}^{ \pm}:=\lim _{n \rightarrow \infty} \frac{1}{\beta\left|\Lambda_{n}\right|} \ln P_{\text {abs }, \Lambda, \gamma, \beta}^{ \pm}\left(\vec{\rho}_{\beta}^{ \pm}\right)
$$


that are continuous in $\beta$, moreover there are constants $c_{b}, \bar{\gamma}$ s.t. for any $\gamma<\bar{\gamma}$ there is a value of $\beta, \beta_{c}(\gamma)$, s.t.

$$
P_{\mathrm{abs}, \gamma, \beta_{c}(\gamma)}^{+}=P_{\mathrm{abs}, \gamma, \beta_{c}(\gamma)}^{-} \quad\left|\beta_{c}^{\mathrm{mf}}-\beta_{c}(\gamma)\right|<c_{b} \gamma^{1 / 2}
$$

The proof is obtained by a continuity argument, and it is based on the following mean field result: there exists an inverse temperature $\beta_{c}^{\mathrm{mf}}$ such that the mean field free energy density satisfies:

$$
\phi_{\beta_{c}^{\mathrm{mf}}}\left(\vec{\rho}_{\beta_{c}^{\mathrm{mf}}}^{+}\right)=\phi_{\beta_{c}^{\mathrm{mf}}}\left(\vec{\rho}_{\beta_{c}^{\mathrm{mf}}}^{-}\right)=\inf _{\vec{\rho} \in S_{Q}} \phi_{\beta_{c}^{\mathrm{mf}}}(\vec{\rho})
$$

and

$$
\left.\frac{d}{d \beta}\left[\phi_{\beta}^{\mathrm{mf}}\left(\vec{\rho}_{\beta}^{+}\right)-\phi_{\beta}^{\mathrm{mf}}\left(\vec{\rho}_{\beta}^{-}\right)\right]\right|_{\beta=\beta_{c}^{\mathrm{mf}}} \neq 0
$$

This result is well known (see [30) and for completeness it is also shown in appendix $\mathrm{A}$

Proof of Theorem 4.5. The proof of existence of the two pressures $P_{\mathrm{abs}, \gamma, \beta_{c}(\gamma)}^{ \pm}$and their continuity in $\beta$ is given in Appendix [C] while the proof of (4.13) is an immediate consequence the following lemma :

Lemma 6.3. There are constants $\kappa$ and $\bar{\gamma}$ such that for any $\gamma<\bar{\gamma}$ and for any $\beta$ such that $\left|\beta-\beta_{c}^{\mathrm{mf}}\right| \leq \gamma^{2 a}$ :

$$
\left|P_{\mathrm{abs}, \gamma, \beta}^{ \pm}+\phi_{\beta}^{\mathrm{mf}}\left(\vec{\rho}_{\beta}^{ \pm}\right)\right| \leq \frac{\kappa}{\beta} \gamma^{1 / 2}
$$

that implies:

$$
\left|P_{\mathrm{abs}, \gamma, \beta}^{+}-P_{\mathrm{abs}, \gamma, \beta}^{-}-\left(\phi_{\beta}^{\mathrm{mf}}\left(\vec{\rho}^{-}\right)-\phi_{\beta}^{\mathrm{mf}}\left(\vec{\rho}^{+}\right)\right)\right|<2 \frac{\kappa}{\beta} \gamma^{1 / 2}
$$

(6.16), (6.14), (6.13), and the continuity in $\beta$ of the pressures prove (4.13) and complete the proof of the Theorem 4.5

PRoOF of Lemma 6.3. The proof of (6.15) could be obtained as a byproduct of a more detailed analysis contained in the next section but since a direct proof is quite shorter we sketch it here. We first prove an upper bound for $P_{\mathrm{abs}, \gamma, \beta}^{ \pm}$.

$$
P_{\mathrm{abs}, \gamma, \beta}^{ \pm}=\lim _{n \rightarrow \infty} \frac{1}{\beta\left|\Lambda_{n}\right|} \ln Z_{\mathrm{abs}, \beta, \Lambda}^{ \pm}\left(\vec{\rho}_{\beta}^{ \pm}\right)
$$


and denoting by $\hat{Z}_{\mathrm{abs}, \beta, \Lambda}^{ \pm}\left(\vec{\rho}_{\beta}^{ \pm}\right)$the abstract partition function with interactions $H_{\gamma, \Lambda}\left(\vec{\xi}_{\Lambda} \mid \vec{\xi}_{\Lambda^{c}}\right)$, we get:

$$
P_{\mathrm{abs}, \gamma, \beta}^{ \pm} \leq \lim _{n \rightarrow \infty} \frac{1}{\beta\left|\Lambda_{n}\right|} \ln \hat{Z}_{\mathrm{abs}, \beta, \Lambda}^{ \pm}\left(\vec{\rho}_{\beta}^{ \pm}\right)+\sup _{\vec{\xi} \in \mathcal{X}^{ \pm}} \sum_{\Delta: \Delta \ni 0}\left|U_{\Delta}^{ \pm}(\vec{\xi})\right|
$$

By (6.6) the last term is bounded as $e^{-\frac{\mathfrak{K}_{\gamma}}{2}}$ and by Theorem 5.1 we have:

$$
P_{\mathrm{abs}, \gamma, \beta}^{ \pm} \leq-\lim _{n \rightarrow \infty} \inf _{\vec{\rho} \in \mathcal{X}_{\Lambda_{n}}} \frac{F_{\beta, \gamma, \Lambda_{n}}\left(\vec{\rho} \mid \vec{\rho}_{\beta}^{ \pm}\right)}{\beta\left|\Lambda_{n}\right|}+c_{d} \gamma^{1 / 2}
$$

we postpone at the end of this section the proof of the following bound that follows by the concavity of the entropy:

$$
P_{\text {abs }, \gamma, \beta}^{ \pm} \leq c_{d} \gamma^{1 / 2}-\lim _{n \rightarrow \infty} \inf _{\vec{\rho} \in \mathcal{X}_{\Lambda_{n}}} \frac{1}{\left|\Lambda_{n}\right|} \int_{\Lambda_{n}} \phi_{\beta}\left(J_{\gamma} *\left(\vec{\rho} \mathbf{1}_{\Lambda_{n}}+\vec{\rho}_{\beta}^{ \pm} \mathbf{1}_{\Lambda_{n}^{c}}\right)\right) d r
$$

where $\mathbf{1}_{A}:=\mathbf{1}_{\{x \in A\}}$. Let

$$
J_{\gamma}^{\left(\ell_{-, \gamma}\right)}(x, y):=\frac{1}{\left(\ell_{-, \gamma}\right)^{d}} \int_{z \in C_{y}^{\ell_{-}, \gamma}} J_{\gamma}(x, z)
$$

where $C_{y}^{\ell_{-, \gamma}}$ is the cube of the partition $\mathcal{D}^{\ell_{-, \gamma}}$ containing the point $y$. Then for any $\vec{s} \in \mathcal{X}^{ \pm}$:

$$
\begin{aligned}
\left\|J_{\gamma} * \vec{s}(r)-\vec{\rho}^{ \pm}\right\|_{\star} \leq\left\|\int J_{\gamma}^{\left(\ell_{-, \gamma}\right)}\left(r, r^{\prime}\right)\left(\vec{s}\left(r^{\prime}\right)-\vec{\rho}^{ \pm}\right)\right\|_{\star} & \\
& +\left\|\int\left[J_{\gamma}^{\left(\ell_{-, \gamma}\right)}\left(r, r^{\prime}\right)-J_{\gamma}(r, r)\right]\left(\vec{s}\left(r^{\prime}\right)-\vec{\rho}^{ \pm}\right)\right\|_{\star}
\end{aligned}
$$

By the assumptions on $J_{\gamma}$ (see (2.2) $)$ the second term is bounded as :

$$
\left\|\int\left[J_{\gamma}^{\left(\ell_{-, \gamma}\right)}\left(r, r^{\prime}\right)-J_{\gamma}\left(r, r^{\prime}\right)\right]\left(\vec{s}\left(r^{\prime}\right)-\vec{\rho}^{ \pm}\right)\right\|_{\star} \leq \kappa_{3} \gamma \ell_{-, \gamma}=\kappa_{3} \gamma^{\alpha}
$$

while the first term, since $\vec{s} \in \mathcal{X} \pm$ and $J^{\left(\ell_{-, \gamma}\right)}\left(r, r^{\prime}\right)$ is constant w.r.t. the second variable in each cube of $\mathcal{D}^{\ell-, \gamma}$ :

$$
\left\|\int J_{\gamma}^{\left(\ell_{-, \gamma}\right)}\left(r, r^{\prime}\right)\left(\vec{s}\left(r^{\prime}\right)-\vec{\rho}^{ \pm}\right)\right\|_{\star} \leq \gamma^{a}
$$

going back to (6.17) we have:

$$
P_{\mathrm{abs}, \gamma, \beta}^{ \pm} \leq c \gamma^{1 / 2}-\inf _{\left\|\vec{s}-\vec{\rho}^{ \pm}\right\|_{\star}<\gamma^{a}+\kappa_{3} \gamma^{\alpha}} \phi_{\beta}(\vec{s})
$$

In appendix $\mathrm{A}$ is shown that $\inf _{\vec{s}} \phi_{\beta_{c}^{\mathrm{mf}}}(\vec{s})=\phi_{\beta_{c}^{\mathrm{mf}}}\left(\bar{\rho}_{\beta_{c}^{\mathrm{mf}}}^{\hat{q}}\right)$, for any $\hat{q} \in\{-1,1, \ldots Q\}$. By continuity for $\left|\beta-\beta_{c}^{\mathrm{mf}}\right|<\gamma^{2 a}, \gamma$ small enough $\phi_{\beta}(\vec{s})$ has $Q+1$ local minima $\vec{\rho}_{\beta}^{\hat{q}}$ s.t. $\left|\hat{\rho}_{\beta}^{\hat{q}}-\hat{\rho}_{\beta_{c}^{\mathrm{mf}}}^{\hat{q}}\right|<c\left|\beta-\beta_{c}^{\mathrm{mf}}\right|<c \gamma^{2 a}$. The are respectively then absolute minimizers in the sets $\left\|\vec{s}-\vec{\rho}^{ \pm}\right\|_{\star}<\gamma^{a}+\kappa_{3} \gamma^{\alpha}$. We then get:

$$
P_{\mathrm{abs}, \gamma, \beta}^{ \pm} \leq c \gamma^{1 / 2}-\phi_{\beta}\left(\vec{\rho}_{\beta}^{ \pm}\right)
$$


We now prove a lower bound for the pressures. By Theorem [5.1 with $\ell=\ell_{0}$, we have for any $\vec{\rho}^{(n)} \in \mathcal{X}_{\Lambda_{n}}^{ \pm}$:

$$
P_{\mathrm{abs}, \gamma, \beta}^{ \pm} \geq-\lim _{n \rightarrow \infty} \frac{F_{\beta, \gamma, \Lambda_{n}}\left(\vec{\rho}^{(n)} \mid \vec{\rho}_{\beta}^{ \pm}\right)}{\beta\left|\Lambda_{n}\right|}-c \gamma^{1 / 2}
$$

and for $\gamma$ small enough the same argument as before shows that $\rho_{\beta}^{ \pm} \mathbf{1}_{\Lambda_{n}} \in \mathcal{X}_{\Lambda_{n}}^{ \pm}$. We then get:

$$
P_{\mathrm{abs}, \gamma, \beta}^{ \pm} \geq-c \gamma^{1 / 2}-\phi_{\beta}\left(\vec{\rho}_{\beta}^{ \pm}\right)
$$

Proof of 6.17):

$$
\begin{aligned}
& F_{\beta, \gamma, \Lambda_{n}}\left(\vec{\rho} \mid \vec{\rho}_{\beta}^{ \pm}\right)=F_{\beta, \gamma}\left(\vec{\rho} \mathbf{1}_{\Lambda_{n}}+\vec{\rho}_{\beta}^{ \pm} \mathbf{1}_{\Lambda_{n}^{c}}\right)-F_{\beta, \gamma}\left(\vec{\rho}_{\beta}^{ \pm} \mathbf{1}_{\Lambda_{n}^{c}}\right)
\end{aligned}
$$

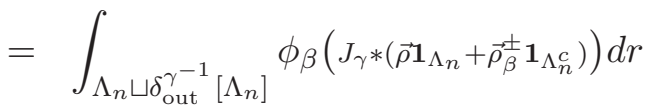

$$
\begin{aligned}
& +\frac{1}{\beta} \int_{\Lambda_{n} \sqcup \delta_{\text {out }}^{\gamma-1}\left[\Lambda_{n}\right]}\left[I\left(J_{\gamma^{*}} *\left(\vec{\rho} \mathbf{1}_{\Lambda_{n}}+\vec{\rho}_{\beta}^{ \pm} \mathbf{1}_{\Lambda_{n}^{c}}\right)\right)-J_{\gamma} * I\left(\vec{\rho} \mathbf{1}_{\Lambda_{n}}+\vec{\rho}_{\beta}^{ \pm} \mathbf{1}_{\Lambda_{n}^{c}}\right)\right] d r
\end{aligned}
$$

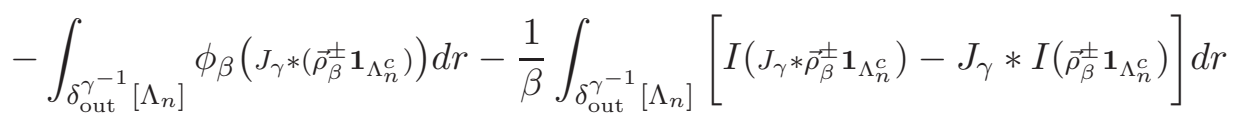

where we have used the fact that $\phi_{\beta}(0)=I(0)=0$. By concavity of $I(\cdot)$ the second term is non negative and since $\left|\phi_{\beta}(\vec{s})\right|$ is bounded in $\vec{s} \in S_{Q}$ we have:

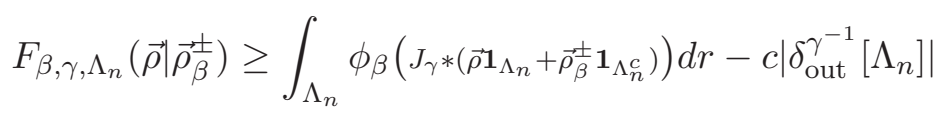

\section{Analysis of the abstract contour models: finite volume corrections to the pressures}

At the critical value of inverse temperature $\beta_{c}(\gamma)$, the theorem 4.5 holds, and the bulk term of the ratios (4.14) is null. In this case, in order to get estimates on Peierls weights it is needed refined a control of the finite volume corrections to the thermodynamical pressure $P_{\mathrm{abs}, \gamma}^{ \pm}$here denoted by $R_{\mathrm{abs}, \Lambda}^{ \pm}$:

$$
R_{\mathrm{abs}, \Lambda}^{ \pm}:=\log Z_{\mathrm{abs}, \beta, \Lambda}^{ \pm}\left(\vec{\rho}^{( \pm)}\right)-\beta|\Lambda| P_{\mathrm{abs}, \gamma}^{ \pm} \quad \lim _{\Lambda \nearrow \mathbb{Z}^{d}} \frac{R_{\mathrm{abs}, \Lambda}^{ \pm}}{|\Lambda|}=0
$$

Let $\Lambda$ a $\mathcal{D}^{\left(\ell_{+, \gamma}\right)}$ bounded region and denoted by $P_{\text {abs }, \gamma, \Lambda}^{ \pm}$the "finite volume pressure": 


$$
P_{\mathrm{abs}, \gamma, \Lambda}^{ \pm}:=\ln Z_{\mathrm{abs}, \beta, \Lambda}^{ \pm}\left(\vec{\rho}^{ \pm}\right)
$$

we prove the following theorem:

Theorem 7.1. There is a constant $c>0$ so that

$$
\left|P_{\mathrm{abs}, \gamma, \Lambda}^{ \pm}-\left\{\beta|\Lambda| P_{\mathrm{abs}, \gamma}^{ \pm}+\frac{\beta}{2} \sum_{\substack{i \in \Lambda \\ j \in \Lambda^{c}}} J_{\gamma}(i, j)\left(\vec{\rho}^{ \pm} \cdot \vec{\rho}^{ \pm}\right)\right\}\right| \leq c \gamma^{1 / 8}\left|\delta_{\mathrm{in}}^{\ell_{+}, \gamma}[\Lambda]\right|
$$

Notice that the leading contribution to $R_{\mathrm{abs}, \Lambda}^{ \pm}$is the finite volume correction to the mean field pressure (with $\rho^{ \pm}$b.c.)

$$
R_{\gamma, \Lambda}^{\mathrm{mf}, \pm}:=\frac{\beta}{2} \sum_{\substack{i \in \Lambda \\ j \in \Lambda^{c}}} J_{\gamma}(i, j)\left(\vec{\rho}^{ \pm} \cdot \vec{\rho}^{ \pm}\right)
$$

The proof of Theorem $[7.1$ is the outcome of two main estimates: the first one is a bound on the decay of correlations and the second step is a small deviation estimate inside "correct regions" to control the contribution coming from regions near the boundary.

\section{Dobrushin interpolations}

In this section we refer to the models with interpolating hamiltonians 4.15)

$$
\hat{h}_{u}^{ \pm}(\vec{\xi}):=u \tilde{H}^{ \pm}\left(\vec{\xi} \mid \vec{\rho}^{ \pm}\right)+(1-u) \mathfrak{H}_{\gamma}^{ \pm}(\vec{\xi})
$$

where $\mathfrak{H}^{ \pm}$are the one body "mean field" Hamiltonians, defined in (4.16).

For any $u \in[0,1]$ we denote by $Z_{\mathrm{abs}, \beta, \Lambda ; u}^{ \pm}$the partition function relative to the hamiltonian $\hat{h}_{u}^{ \pm}(\vec{\xi})$. In particular, $Z_{\text {abs, } \beta, \Lambda ; 0}^{ \pm}$corresponds to the interpolating one-body hamiltonian $\mathfrak{H}_{\gamma, \Lambda}^{ \pm}(\vec{\xi})$ in (4.16).

We recall also the expression of the [finite volume] pressure $P_{\mathrm{abs}, \gamma, \Lambda}^{ \pm}$(4.17)) in terms of correlation functions w.r.t. the measures $\hat{\mu}_{\mathrm{abs}, \Lambda ; u}^{ \pm}$:

$$
\begin{aligned}
P_{\mathrm{abs}, \gamma, \Lambda}^{ \pm} & :=\frac{1}{\beta|\Lambda|} \ln Z_{\mathrm{abs}, \beta, \Lambda ; 1}^{ \pm}\left(\vec{\rho}^{ \pm}\right) \\
& =\frac{1}{\beta|\Lambda|} \ln Z_{\mathrm{abs}, \beta, \Lambda ; 0}^{ \pm}-\frac{1}{|\Lambda|} \int_{0}^{1} d u\left\langle\tilde{H}_{\Lambda}^{ \pm}\left(\vec{\xi}_{\Lambda}^{\prime} \mid \vec{\rho}^{ \pm}\right)-\mathfrak{H}_{\Lambda}^{ \pm}\left(\vec{\xi}_{\Lambda}^{\prime}\right)\right\rangle_{\hat{\mu}_{\mathrm{abs}, \Lambda ; u}^{ \pm}}
\end{aligned}
$$

and prove the following proposition: 
Proposition 7.2. The exist $g_{\gamma, i, \Lambda}, g_{\gamma, i}: \mathbb{Z}^{d} \rightarrow \mathbb{R}$ :

$$
\begin{aligned}
& {\left[\tilde{H}_{\Lambda}^{ \pm}\left(\vec{\xi}_{\Lambda}^{\prime} \mid \vec{\rho}^{ \pm}\right)-\mathfrak{H}_{\Lambda}^{ \pm}\left(\vec{\xi}_{\Lambda}^{\prime}\right)\right]=\sum_{i \in \Lambda} g_{\gamma, i, \Lambda}} \\
& \lim _{\Lambda \nearrow \mathbb{R}^{d}} g_{\gamma, i, \Lambda}=g_{\gamma, i}
\end{aligned}
$$

PROOF.

$$
\tilde{H}_{\Lambda}^{ \pm}\left(\vec{\xi}_{\Lambda} \mid \vec{\rho}^{ \pm}\right)-\mathfrak{H}_{\Lambda}^{ \pm}\left(\vec{\xi}_{\Lambda}\right)=\frac{1}{2} \sum_{i \in \Lambda} \overrightarrow{\delta \xi}_{i} \sum_{\substack{j \in \Lambda \\ j \neq i}} J_{\gamma}(i, j) \overrightarrow{\delta \xi}_{j}+\mathcal{H}_{\gamma, \Lambda}^{ \pm}\left(\vec{\xi}_{\Lambda}\right)
$$

Recalling (6.4):

$$
\mathcal{H}_{\Lambda}^{ \pm}(\vec{\xi})=\sum_{\Delta \sqsubset \Lambda} U_{\Delta}^{ \pm}(\vec{\xi})=\sum_{i \in \Lambda} \sum_{\substack{\Delta \sqsubset \Lambda \\ \Delta \ni i}} \frac{1}{|\Delta|} U_{\Delta}^{ \pm}(\vec{\xi})
$$

Defining:

$$
g_{\gamma, i, \Lambda}(\vec{\xi}):=\frac{1}{2} \overrightarrow{\delta \xi_{i}} \sum_{\substack{j \in \Lambda: \\ j \neq i}} J_{\gamma}(i, j) \overrightarrow{\delta \xi}_{j}+\sum_{\substack{\Delta \ni i \\ \Delta \sqsubset \Lambda}} \frac{1}{|\Delta|} U_{\Delta}^{ \pm}(\vec{\xi})
$$

we can write:

$$
\tilde{H}_{\Lambda}^{ \pm}\left(\vec{\xi}_{\Lambda} \mid \vec{\rho}^{ \pm}\right)-\mathfrak{H}_{\Lambda}^{ \pm}\left(\vec{\xi}_{\Lambda}\right)=\sum_{i \in \Lambda} g_{\gamma, i, \Lambda}(\vec{\xi})
$$

Recalling (6.6), the limit (17.6) exists:

$$
\lim _{\Lambda \nearrow \mathbb{R}^{d}} g_{\gamma, i, \Lambda}(\vec{\xi})=-\frac{1}{2} \overrightarrow{\delta \xi}_{i} \sum_{j: j \neq i} J_{\gamma}(i, j) \overrightarrow{\delta \xi} \xi_{j}+\sum_{\Delta \ni i} \frac{1}{|\Delta|} U_{\Delta}^{ \pm}(\vec{\xi})=: g_{\gamma, i}(\vec{\xi})
$$

Postponing the proof of the existence of the limit of the Gibbs measure $\tilde{\mu}_{\mathrm{abs}, \Lambda ; u}^{ \pm}$when $\Lambda \nearrow \mathbb{R}^{d}$ and denoting it by $\tilde{\mu}_{\mathrm{abs} ; u}^{ \pm}$, the limit of (4.17) gives:

$$
P_{\mathrm{abs}, \gamma}^{ \pm}=P_{\mathrm{abs}, \gamma, 0}^{ \pm}-\int_{0}^{1} d u\left\langle g_{\gamma, 0}\right\rangle_{\tilde{\mu}_{\mathrm{abs} ; u}^{ \pm}}
$$

and (4.17) can be rewritten as:

$$
\begin{aligned}
P_{\mathrm{abs}, \gamma, \Lambda}^{ \pm}=P_{\mathrm{abs}, \gamma}^{ \pm}+ & {\left[\frac{1}{\beta|\Lambda|} \ln Z_{\mathrm{abs}, \beta, \Lambda ; 0}^{ \pm}-P_{\mathrm{abs}, \gamma, 0}^{ \pm}\right] } \\
& -\int_{0}^{1} d u\left[\frac{1}{|\Lambda|} \sum_{i \in \Lambda}\left\langle g_{\gamma, i, \Lambda}\right\rangle_{\tilde{\mu}_{\mathrm{abs}, \Lambda ; u}^{ \pm}}-\left\langle g_{\gamma, 0}\right\rangle_{\tilde{\mu}_{\mathrm{abs} ; u}^{ \pm}}\right]
\end{aligned}
$$

The finite volume corrections are then given by:

$$
R_{\mathrm{abs}, \Lambda}^{ \pm}=R_{\mathrm{abs}, \Lambda, 0}^{ \pm}-\beta \int_{0}^{1} d u \sum_{i \in \Lambda}\left[\left\langle g_{\gamma, i, \Lambda}\right\rangle_{\tilde{\mu}_{\mathrm{abs}, \Lambda ; u}^{ \pm}}-\left\langle g_{\gamma, i}\right\rangle_{\tilde{\mu}_{\mathrm{abs} ; u}^{ \pm}}\right]
$$


where

$$
R_{\mathrm{abs}, \Lambda, 0}^{ \pm}:=\ln Z_{\mathrm{abs}, \beta, \Lambda ; 0}^{ \pm}-\beta|\Lambda| P_{\mathrm{abs}, \gamma, 0}^{ \pm}
$$

In appendix $\mathrm{D}$ it is proven that:

$$
R_{\mathrm{abs}, \Lambda, 0}^{ \pm}=R_{\gamma, \Lambda}^{\mathrm{mf}, \pm}
$$

and then the proof of the Theorem 7.1 follows by estimating the remaining terms in (17.10). In the next subsections we will prove that there are positive constant $c, \omega$ such that:

1)

$$
\left|\left\langle g_{\gamma, i, \Lambda}\right\rangle_{\tilde{\mu}_{\mathrm{abs}, \Lambda ; u}^{ \pm}}-\left\langle g_{\gamma, i}\right\rangle_{\tilde{\mu}_{\mathrm{abs} ; u}^{ \pm}}\right| \leq c e^{-\omega \gamma \operatorname{dist}(i, \Lambda)}
$$

2)

$$
\sum_{i \in \delta_{\text {in }}^{\ell+,}[\Lambda]}\left\{\left|\left\langle g_{\gamma, i}\right\rangle_{\tilde{\mu}_{\text {abs } ; u}^{ \pm}}\right|+\left|\left\langle g_{\gamma, i, \Lambda}\right\rangle_{\tilde{\mu}_{\text {abs }, \Lambda ; u}^{ \pm}}\right|\right\} \leq c \gamma^{1 / 8}\left|\delta_{\text {in }}^{\ell+, \gamma}[\Lambda]\right|
$$

The proof of (7.12) and the existence of the limit of $\hat{\mu}_{\mathrm{abs}, \Lambda ; u}^{ \pm}$when $\Lambda \nearrow \mathbb{R}^{d}$ follow by the proof of the exponentially decay of the correlations given in next subsection, while the estimate (17.13) follows from the small deviations estimates proved in the last subsection.

Proof of Theorem 17.1. Collecting (7.10), (7.11), (7.12), (7.13) and using the last one for estimating the contribution to the correction coming from the boundary and (7.12) for estimating the contribution to the corrections coming from the volume inside, we get (7.1). Details are omitted.

7.1. Decay of the correlations. In order to prove (7.12) we state the following Theorem:

Theorem 7.3. There are $c$ and $\omega$ positive so that for $u \in(0,1)$ and for any bounded

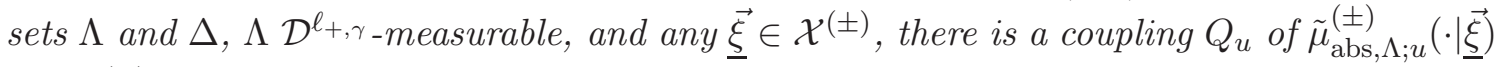
and $\tilde{\mu}_{\mathrm{abs} ; u}^{( \pm)}(\cdot)$ such that

$$
Q_{u}\left(\vec{\xi}_{\Delta} \neq \vec{\xi}_{\Delta}^{\prime}\right) \leq c|\Delta| e^{-\omega \gamma \operatorname{dist}\left(\Delta, \Lambda^{c}\right)}
$$

It follows that:

Corollary 7.4. For any $u \in[0,1]$ there is a unique DLR measure $\tilde{\mu}_{\mathrm{abs} ; u}^{ \pm}$with hamiltonian $h_{u}^{ \pm}$and for any local function $f$ with support in $\Delta$ :

$$
\lim _{\Lambda \nearrow \mathbb{Z}^{d}}\langle f\rangle_{\tilde{\mu}_{\mathrm{abs}, \Lambda ; u}^{ \pm}}=\langle f\rangle_{\tilde{\mu}_{\mathrm{abs} ; u}^{ \pm}}
$$


and, for any $\Delta \sqsubset \Lambda$, there are positive constants $c, \omega$ :

$$
\left|\langle f\rangle_{\tilde{\mu}_{\mathrm{abs}, \Lambda ; u}^{ \pm}}-\langle f\rangle_{\tilde{\mu}_{\mathrm{abs} ; u}^{ \pm}}\right| \leq c|\Delta| \sup _{x \in \Delta}\{f(x)\} \quad e^{-\omega \gamma \operatorname{dist}\left(\Delta, \Lambda^{c}\right)}
$$

Corollary 7.4 applied to our case, proves inequality (7.12).

Proof. The proof of Theorem 7.3 requires an extension of the Dobrushin high temperature uniqueness Theorem. In reference [3], the Dobrushin uniqueness criterium is extended to the case when the "classical Dobrushin condition" is not satisfied uniformly in the boundary conditions, but only for "most of the configurations". This is the case for the abstract contour models, where, due to the constrain on the space of the configurations $\mathcal{X}^{ \pm}$, the Vasenstein distance between two Gibbs measures on a single spin, with different boundary conditions is not small uniformly in all the boundary conditions.

In reference [3] it is shown that, provided two main assumptions are verified, the Dobrushin criterium can be extended to cover such a case. A further assumption provides an exponential decay for the correlations. Two other assumptions trivially hold in our case and are not reported here. We refer to [3] and the Theorem [7.3 will be proved through the demonstration that the two abstract models $\tilde{\mu}_{\text {abs;u }}^{( \pm)}\left(d \vec{\xi}^{\prime}\right)$ fulfill the requirements of the extended Dobrushin criteron.

\subsubsection{First requirement:}

First, e need to prove that for any $i \in \mathbb{Z}^{d}$ there is a measurable set $G_{i}^{ \pm} \sqsubset \mathcal{X}^{ \pm}$depending only on $\left\{\underline{\vec{\xi}}_{j}, j \in C_{i}^{\ell_{-, \gamma}} \backslash i\right\}$, such that there exists $b(i, j)$ with the following properties:

$$
\begin{aligned}
& b(i, j) \geq 0 ; b(i, i)=0 \\
& \sup _{i \in \mathbb{Z}^{d}} \sum_{j \in C_{i}^{\ell-\gamma}} b(i, j)<\delta<1 \\
& \mathcal{R}\left(\mu_{\mathrm{abs}, i ; u}^{ \pm}\left(\vec{\xi}_{i} \mid \vec{\xi}\right), \mu_{\mathrm{abs}, i ; u}^{ \pm}\left(\overrightarrow{\vec{\xi}_{i}} \mid \underline{\vec{\xi}}\right)\right) \leq \sum_{j} b(i, j) \operatorname{dist}\left(\underline{\vec{\xi}}_{j}, \underline{\xi}_{j}\right) \quad \text { for any } \underline{\vec{\xi}}, \underline{\vec{\xi}} \in G_{i}
\end{aligned}
$$

where $\operatorname{dist}\left(\vec{\xi}_{j}, \vec{\xi}_{j}^{\prime}\right)$ is a distance defined on the configuration space and

$\mathcal{R}\left(\mu_{\mathrm{abs}, i ; u}^{ \pm}\left(\vec{\xi}_{i} \mid \underline{\vec{\xi}}\right), \mu_{\mathrm{abs}, i ; u}^{ \pm}\left(\vec{\xi}_{i} \mid \underline{\xi^{\prime}}\right)\right)$ is the associated Vaserstein distance. Here, we consider the following distance between configurations:

$$
\operatorname{dist}\left(\vec{\xi}_{i}, \vec{\xi}_{i}^{\prime}\right):=\frac{1}{2} \sum_{i}\left|\xi_{i}(i)-\xi_{i}^{\prime}(i)\right|
$$

and define $G_{i}$ as :

$$
G_{i}^{ \pm}:=\left\{\vec{\xi} \in \mathcal{X}^{( \pm)}: \vec{\xi}^{(i, q)} \in \mathcal{X}^{( \pm)} \forall q\right\}
$$


where we have denoted

$$
\vec{\xi}_{j}^{(i, q)}= \begin{cases}\vec{\xi}_{j} & j \neq i \\ \vec{u}_{q} & j=i\end{cases}
$$

Remark: $G_{i}^{ \pm}$is the set of configurations $\vec{\xi}$ which belong to $\mathcal{X}^{( \pm)}$independently of the value of $\vec{\xi}_{i}$ and is measurable on $\vec{\xi}_{i c}$. When $\underline{\vec{\xi}}, \underline{\xi}$ are not in $G_{i}^{ \pm}$, the probability measures for $\vec{\xi}_{i}$, have support on a strict subset of $\Omega$ and the Vaserstein distance can be larger than the bound in (4.18).

Theorem 7.5. There are $\bar{\gamma}, \varsigma$, so that for $\gamma<\bar{\gamma}$, and for any $\beta:\left|\beta-\beta_{c}^{\mathrm{mf}}\right|<c_{b} \gamma^{\frac{1}{2}}$, there exists $b(i, j)$ satisfying the relations (4.18) with $G_{i}$ as in (4.20). $b(i, j)$ has the expression:

$$
b(i, j)=r\left[J_{\gamma}(i, j)+3^{d} e^{-\frac{\mathfrak{k} \gamma}{2} N_{i, j}}\right]
$$

where $r<1$, and $N_{i, j}$ as in (6.8) and satisfying the bound [6.9])

Proof. The Vaserstein distance between the two measures $\mu_{i}\left(\vec{\xi}_{i} \mid \vec{\xi}_{1}\right), \mu_{i}\left(\vec{\xi}_{i} \mid \vec{\xi}_{2}\right)$ is defined as

$$
\mathcal{R}\left(\mu_{i}\left(\vec{\xi}_{i} \mid \vec{\xi}_{1}\right), \mu_{i}\left(\vec{\xi}_{i} \mid \vec{\xi}_{2}\right)\right):=\inf _{\amalg} E_{\amalg}\left(\operatorname{dist}\left(\vec{\xi}_{i}, \vec{\xi}_{i}^{\prime}\right)\right)
$$

where the infimum is taken over all couplings between $\mu_{i}\left(\vec{\xi}_{i} \mid \vec{\xi}_{1}\right), \mu_{i}\left(\vec{\xi}_{i} \mid \vec{\xi}_{2}\right)$. Recalling (4.19), the infimum is realized on the couplings which have the maximal mass on the diagonal

$$
\amalg\left(\vec{\xi}_{i}=\vec{\xi}_{i}^{\prime}=\vec{u}_{q}\right)=\min \left\{\mu_{i}\left(\vec{u}_{q} \mid \vec{\xi}_{1}\right), \mu_{i}\left(\vec{u}_{q} \mid \vec{\xi}_{2}\right)\right\}
$$

Let $\amalg$ be such a coupling for $\mu_{\text {abs }, i ; u}\left(\vec{\xi}_{i} \mid \vec{\xi}_{1}\right), \mu_{\text {abs }, i ; u}\left(\vec{\xi}_{i} \mid \vec{\xi}_{2}\right)$. We have:

$$
\begin{aligned}
\mathcal{R}\left(\mu_{\mathrm{abs}, i ; u}\left(\vec{\xi}_{i} \mid \vec{\xi}_{1}\right), \mu_{\mathrm{abs}, i ; u}\left(\vec{\xi}_{i} \mid \vec{\xi}_{2}\right)\right) & =E_{\amalg}\left(\operatorname{dist}\left(\vec{\xi}_{i}, \vec{\xi}_{i}^{\prime}\right)\right)=\amalg\left(\vec{\xi}_{i} \neq \vec{\xi}_{i}^{\prime}\right) \\
& =1-\sum_{q} \min \left\{\mu_{\mathrm{abs}, i ; u}\left(\vec{u}_{q} \mid \vec{\xi}_{1}\right), \mu_{\mathrm{abs}, i ; u}\left(\vec{u}_{q} \mid \vec{\xi}_{2}\right)\right\} \\
& =\frac{1}{2} \sum_{q}\left(\mu_{\mathrm{abs}, i ; u}\left(\vec{u}_{q} \mid \vec{\xi}_{1}\right)+\mu_{\mathrm{abs}, i ; u}\left(\vec{u}_{q} \mid \vec{\xi}_{2}\right)\right. \\
& =\frac{1}{2} \sum_{q}\left|\mu_{\mathrm{abs}, i ; u}\left(\vec{u}_{q} \mid \vec{\xi}_{1}\right)-\mu_{\mathrm{abs}, i ; u}\left(\vec{u}_{q} \mid \vec{\xi}_{2}\right)\right|
\end{aligned}
$$

Since $\underline{\vec{\xi}}$ and $\underline{\xi}$ are in $G_{i}$, the constraint of being in $\mathcal{X}^{ \pm}$does not affect the values of $\vec{\xi}_{i}$, and the conditional measures $\mu_{\mathrm{abs}, i ; u}^{ \pm}(\cdot \mid \vec{\xi})$ are given by the Gibbs conditional measures. Using (6.10), we get, 


$$
\begin{aligned}
\mathcal{R}\left(\mu_{\mathrm{abs}, i ; u}^{ \pm}\left(\overrightarrow{\xi_{i}} \mid \underline{\vec{\xi}}\right), \mu_{\mathrm{abs}, i ; u}^{ \pm}\left(\vec{\xi}_{i} \mid \underline{\xi}^{\prime}\right)\right) & =\frac{1}{2} \sum_{q}\left|\frac{e^{\beta k_{q}^{ \pm ; u}(\underline{\vec{\xi}})}}{\sum_{p=1}^{Q} e^{\beta k_{p}^{ \pm ; u}(\underline{\vec{\xi}})}}-\frac{e^{\beta k_{q}^{ \pm ; u}\left(\vec{\xi}^{\prime}\right)}}{\sum_{p=1}^{Q} e^{\beta k_{p}^{ \pm ;}\left(\underline{\vec{\xi}}^{\prime}\right)}}\right| \\
& =\frac{1}{2} \sum_{q}\left|g_{q}\left(\vec{k}^{ \pm ; u}(\underline{\vec{\xi}})\right)-g_{q}\left(\vec{k}^{ \pm ; u}\left(\vec{\xi}^{\prime}\right)\right)\right|
\end{aligned}
$$

Where $\vec{g}(\cdot)$ is defined in (A.12) and $\vec{k}_{i}^{ \pm ; u}(\underline{\vec{\xi}}) \equiv \vec{k}_{i}^{ \pm ; u}\left(\underline{\vec{\xi}}_{i^{c}}\right)$ is the vector

$$
\begin{aligned}
\vec{k}_{i}^{ \pm}(\underline{\xi}) & :=u \overrightarrow{\mathcal{L}}_{\gamma}(\vec{\xi} ; i)+(1-u) \overrightarrow{\mathcal{L}}_{\gamma}\left(\vec{\rho}^{ \pm} ; i\right)-u \sum_{q}\left(\mathcal{H}^{ \pm}\left(\underline{\xi}^{(i, q)}\right)-\mathcal{H}^{ \pm}\left(\vec{\xi}_{i^{c}}\right)\right) \vec{u}_{q} \\
& =u \sum_{j \neq i} J_{\gamma}(i, j) \vec{\xi}_{j}+(1-u) \sum_{j \neq i} J_{\gamma}(i, j) \vec{\rho}_{j}^{ \pm}-u \sum_{q} \sum_{\Delta \ni i} U_{\Delta}^{ \pm}\left(\vec{\xi}^{(i, q)}\right) \vec{u}_{q}
\end{aligned}
$$

Using (6.6), we have:

$$
\lim _{\gamma \rightarrow 0} \sup _{\vec{\xi} \in G_{i}}\left\|\vec{k}^{ \pm}(\underline{\vec{\xi}})-\vec{\rho}^{ \pm}\right\| \leq \lim _{\gamma \rightarrow 0}\left(c \gamma^{a}+\sum_{\Delta \ni i} Q\left\|U_{\Delta}^{ \pm}(\cdot)\right\|_{\infty}\right)=0
$$

Hence, for $\gamma$ small enough, we can use theorem A.3 and get

$$
\mathcal{R}\left(\mu_{\mathrm{abs}, i ; u}^{ \pm}\left(\vec{\xi}_{i} \mid \underline{\vec{\xi}}\right), \mu_{\mathrm{abs}, i ; u}^{ \pm}\left(\vec{\xi}_{i} \mid \underline{\vec{\xi}}\right)\right) \leq \frac{1}{2}\left(1-\frac{1}{2 Q}\right) \sum_{q}\left|k_{q}^{ \pm ; u}(\underline{\vec{\xi}})-k_{q}^{ \pm ; u}(\underline{\vec{\xi}})\right|
$$

On the other hand, we have the following bound for all $\Delta \ni x$

$$
\left|U_{\Delta}^{ \pm}\left(\vec{\xi}^{(i, q)}\right)-U_{\Delta}^{ \pm}\left(\vec{\xi}^{(i, q)}\right)\right| \leq 2\left\|U_{\Delta}^{ \pm}(\cdot)\right\|_{\infty} \sum_{j \in \Delta \backslash i} \operatorname{dist}\left(\underline{\vec{\xi}}_{j}, \underline{\xi}_{j}\right)
$$

and thus

$$
\sum_{q}\left|k_{q}^{ \pm ; u}(\underline{\vec{\xi}})-k_{q}^{ \pm ; u}(\underline{\vec{\xi}})\right| \leq 2 \sum_{j \neq i}\left(u J_{\gamma}(i, j)+\sum_{\Delta \ni i, j} Q\left\|U_{\Delta}^{ \pm}(\cdot)\right\|_{\infty}\right) \operatorname{dist}\left(\underline{\vec{\xi}}_{j}, \underline{\xi}_{j}\right)
$$

Recalling (6.7) we bound the last term in (7.20) as:

$$
\sum_{\Delta \ni i, j}\left\|U_{\Delta}^{ \pm}(\cdot)\right\|_{\infty} \leq \sum_{\Delta \ni i, j} e^{-\left(\frac{\mathfrak{K}_{\gamma}}{2}-b\right) N_{\Delta}} \leq 3^{d} e^{-\left(\frac{\mathfrak{K}_{\gamma}}{2}\right) N_{i, j}} \leq 3^{d} e^{-\frac{\mathfrak{K}_{\gamma}}{2} N_{i, j}}
$$

Since $\mathfrak{K}_{\gamma}=c \gamma^{-(1-\alpha) d+2 a}$,

$$
\lim _{\gamma \rightarrow 0} \sum_{\Delta \ni i, j} Q\left\|U_{\Delta}^{ \pm}(\cdot)\right\|_{\infty}=0
$$

hence, we take for all $u$,

$$
b(i, j):=\left(1-\frac{1}{2 Q}\right)\left(u J_{\gamma}(i, j)+3^{d} e^{-\frac{\mathfrak{K}_{\gamma}}{2} N_{i, j}}\right)
$$


We have

$$
\lim _{\gamma \rightarrow 0} \sup _{i \in \mathbb{Z}^{d}} \sum_{j \in C_{i}^{\ell_{-}, \gamma} \backslash i} b(i, j)=0
$$

so that (4.18) holds for all $\gamma$ small enough.

\subsubsection{Second Requirement:}

We need now a bound on the probability of $G_{i}^{ \pm c}$, where $G_{i}^{ \pm}$is defined in (4.20). Here we deal with a bounded state space and the required bound may be written as:

$$
\sup _{\overrightarrow{\xi^{*}} \in \mathcal{X}^{ \pm}} \mu_{\mathrm{abs}, D, u}^{( \pm)}\left(G_{i}^{ \pm c} \mid \vec{\xi}^{*}\right) \leq e^{-c \gamma^{2 a} \ell_{-, \gamma}^{d}}
$$

$c$ a positive constant and $D=C_{i}^{\ell_{-, \gamma}}$. The proof of (7.24) requires a Peierls estimate inside the restricted set $\mathcal{X}^{ \pm}$. Let $\zeta^{\prime}>0$, we define the "bad" set

$$
\mathcal{A}_{i}^{ \pm}:=\left\{\vec{\xi} \in \mathcal{X}^{( \pm)}:\left\|\vec{\xi}^{\left(\ell_{-, \gamma}\right)}(i)-\vec{\rho}^{ \pm}\right\|_{\star}>\left(1-\zeta^{\prime}\right) \gamma^{a}\right\}
$$

Notice that for any $\zeta^{\prime}>0$ and $\gamma$ small enough, we have for all $i$

$$
G_{i}^{ \pm c} \sqsubset \mathcal{A}_{i}^{ \pm}
$$

so that $\mathcal{A}_{i}^{ \pm}$does not depend on $\vec{\xi}_{i}$, and we have

$$
\sup _{\vec{\xi}^{*} \in \mathcal{X}^{ \pm}} \mu_{\mathrm{abs}, D, u}^{( \pm)}\left(G_{i}^{ \pm c} \mid \vec{\xi}^{*}\right) \leq \sup _{\vec{\xi}^{*} \in \mathcal{X}^{ \pm}} \mu_{\mathrm{abs}, D, u}^{( \pm)}\left(\mathcal{A}_{i}^{ \pm} \mid \vec{\xi}^{*}\right)
$$

Let

$$
\begin{aligned}
F_{\gamma, D, u}:= & -\frac{u}{2} \int_{D \times D} d r d r^{\prime} \quad J_{\gamma}\left(r, r^{\prime}\right) \vec{\rho}_{D}(r) \cdot \vec{\rho}_{D}\left(r^{\prime}\right)-(1-u) \int_{D} d r \vec{\rho}_{D}(r) \cdot \vec{\rho}^{ \pm} \\
& -\int_{D \times D^{c}} d r \quad J_{\gamma}\left(r, r^{\prime}\right) \vec{\rho}_{D}(r) \cdot \vec{\rho}_{D^{c}}\left(r^{\prime}\right)+\frac{1}{\beta} \int_{D} d r \vec{\rho}_{D}(r) \cdot \ln \vec{\rho}_{D}(r)
\end{aligned}
$$

The proof of the bound (7.24) is thus based on the following proposition whose proof is given at the end of subsection 7.2

Proposition 7.6. There is a constant $c>0$ so that for all $\gamma$ small enough, any $x$ :

$$
\begin{gathered}
\sup _{\vec{\xi}^{*} \in \mathcal{X}^{( \pm)}} \ln \mu_{\mathrm{abs}, D, u}\left(\mathcal{A}^{ \pm} \mid \vec{\xi}^{*}\right) \leq \inf _{\vec{\rho}_{D^{c}} \in \mathcal{X}_{D^{c}}^{ \pm}}\left(\inf _{\substack{\vec{\rho}_{D} \in \mathcal{X}_{D}^{ \pm},\left|\vec{\rho}^{\left(\ell_{-, \gamma}\right)}(r)-\vec{\rho}^{ \pm}\right|>\left(1-\zeta^{\prime}\right) \zeta}} F_{\gamma, D, u}\left(\vec{\rho}_{D} \mid \vec{\rho}_{D^{c}}\right)\right. \\
\left.-\inf _{\vec{\rho}_{D} \in \mathcal{X}_{D}^{ \pm}} F_{\gamma, D, u}\left(\vec{\rho}_{D} \mid \vec{\rho}_{D^{c}}\right)\right)+c \gamma^{1 / 2} \ell_{-, \gamma}^{d}
\end{gathered}
$$


where, by an abuse of notation, we have denoted by the same symbol $\mathcal{X}^{ \pm}$, the restricted ensemble:

$$
\mathcal{X}^{ \pm}:=\left\{\vec{\rho} \in L^{\infty}\left(\mathbb{R}^{d}, S_{Q}\right):\left\|\vec{\rho}^{\left(\ell_{-, \gamma}\right)}(r)-\vec{\rho}^{ \pm}\right\|_{\star} \leq \gamma^{a}, \quad \forall r \in \mathbb{R}^{d}\right\}
$$

and denote by $\mathcal{X}_{\Lambda}^{ \pm}$the above expression (7.26) when the constrain is imposed on $\Lambda$, with $\Lambda \sqsubset \mathbb{R}^{d}$, a $\mathcal{D}^{\left(\ell_{-, \gamma}\right)}$-measurable set.

Proof. Using a result similar to Theorem 5.1 but with a slightly different functional, one gets for all $\vec{\xi}^{*} \in \mathcal{X}^{( \pm)}$

$$
\begin{aligned}
& \ln \mu_{\mathrm{abs}, D, u}\left(\mathcal{A}^{ \pm} \mid \vec{\xi}^{*}\right)=\ln Z_{\mathrm{abs}, D, u}\left(\mathcal{A}^{ \pm} \mid \vec{\xi}^{*}\right)-\ln Z_{\mathrm{abs}, D, u}\left(\vec{\xi}^{*}\right) \\
& \leq \quad \inf _{\vec{\rho}_{D} \in \mathcal{X}_{D}^{ \pm},} F_{\gamma, D, u}\left(\vec{\rho}_{D} \mid\left(\vec{\xi}^{*}\right)^{\left(\ell_{0}\right)}\right)-\inf _{\vec{\rho}_{D} \in \mathcal{X}_{D}^{ \pm}} F_{\gamma, D, u}\left(\vec{\rho}_{D} \mid\left(\vec{\xi}^{*}\right)^{\left(\ell_{0}\right)}\right)+c \gamma^{-1} \ell_{0}|D| \\
& \left.\mid \vec{\rho}^{(\ell-, \gamma}\right)(r)-\vec{\rho}^{ \pm} \mid>\left(1-\zeta^{\prime}\right) \zeta \\
& \leq-\inf _{\vec{\rho}_{D^{c}} \in \mathcal{X}_{D^{c}}^{ \pm}}\left(\inf _{\substack{\vec{\rho}_{D} \in \mathcal{X}_{D}^{ \pm},\left|\vec{\rho}^{(\ell-, \gamma)}(r)-\vec{\rho}^{ \pm}\right|>\left(1-\zeta^{\prime}\right) \zeta}} F_{\gamma, D, u}\left(\vec{\rho}_{D} \mid \vec{\rho}_{D^{c}}\right)-\inf _{\vec{\rho}_{D} \in \mathcal{X}_{D}^{ \pm}} F_{\gamma, D, u}\left(\vec{\rho}_{D} \mid \vec{\rho}_{D^{c}}\right)\right)+c \gamma^{1 / 2} \ell_{-, \gamma}^{d}
\end{aligned}
$$

We need now an estimate for the right hand side of the previous equation. We define:

$$
F_{\gamma, D, u}^{0}\left(\vec{\rho}_{D} \mid \vec{\rho}_{D^{c}}\right):=-\int_{D} d r \quad\left(\overrightarrow{\mathcal{L}}^{u}\left(r, \vec{\rho}_{D^{c}}\right) \cdot \vec{\rho}_{D}-\frac{1}{\beta} \vec{\rho}_{D} \cdot \ln \vec{\rho}_{D}\right)
$$

where $\overrightarrow{\mathcal{L}^{u}}\left(r, \vec{\rho}_{D^{c}}\right)$ is the external field:

$$
\overrightarrow{\mathcal{L}}^{u}\left(r, \vec{\rho}_{D^{c}}\right):=(1-u) \vec{\rho}^{ \pm}+u \int_{D^{c}} J_{\gamma}\left(r, r^{\prime}\right) \vec{\rho}_{D^{c}}\left(r^{\prime}\right) d r^{\prime}
$$

Since $F_{\gamma, \beta, D}^{0}\left(\vec{\rho}_{D} \mid \vec{\rho}_{D^{c}}\right)$ differs from $F_{\gamma, \beta, D}\left(\vec{\rho}_{D} \mid \vec{\rho}_{D^{c}}\right)$ by the self interaction energy, which is bounded proportionally to $|D|^{2}$, there is a constant $c^{\prime}>0$ such that:

$$
\left|F_{\gamma, D, u}\left(\vec{\rho}_{D} \mid \vec{\rho}_{D^{c}}\right)-F_{\gamma, D, u}^{0}\left(\vec{\rho}_{D} \mid \vec{\rho}_{D^{c}}\right)\right| \leq c^{\prime} u \gamma^{d}|D|^{2}
$$

$F_{\gamma, D}^{0}\left(\vec{\rho}_{D} \mid \vec{\rho}_{D^{c}}\right)$ is a convex functional on $L^{\infty}\left(D, S_{Q}\right)$, and has thus a unique minimizer that we denote by $\vec{\rho}^{*}\left(r ; \vec{\rho}_{D^{c}}\right) \equiv \vec{\rho}^{*}(r)$, whose components are given by:

$$
\rho_{k}{ }^{*}(r)=\frac{e^{\beta \mathcal{L}_{k}^{u}\left(r, \vec{\rho}_{D^{c}}\right)}}{\sum_{l} e^{\beta \mathcal{L}_{l}^{u}\left(r, \vec{\rho}_{D^{c}}\right)}}
$$


We need to evaluate the difference:

$$
\begin{aligned}
F_{\gamma, D, u}^{0}\left(\vec{\rho}_{D} \mid \vec{\rho}_{D^{c}}\right)- & F_{\gamma, D, u}^{0}\left(\vec{\rho}_{D}^{*} \mid \vec{\rho}_{D^{c}}\right)= \\
\int_{D} d r^{\prime} & \left(-\vec{\rho}_{D}\left(r^{\prime}\right) \cdot \overrightarrow{\mathcal{L}}^{u}\left(r, \vec{\rho}_{D^{c}}\right)+\frac{1}{\beta} \vec{\rho}_{D}\left(r^{\prime}\right) \cdot \ln \vec{\rho}_{D}\left(r^{\prime}\right)\right)- \\
& \left(-\vec{\rho}_{D}^{*}\left(r^{\prime}\right) \cdot \overrightarrow{\mathcal{L}}^{u}\left(r, \vec{\rho}_{D^{c}}\right)+\frac{1}{\beta} \vec{\rho}_{D}^{*}\left(r^{\prime}\right) \cdot \ln \vec{\rho}_{D}^{*}\left(r^{\prime}\right)\right)
\end{aligned}
$$

Using (17.29), we write $\overrightarrow{\mathcal{L}^{u}}\left(r, \vec{\rho}_{D^{c}}\right)$ in terms of $\vec{\rho}^{*}$ and get:

$$
F_{\gamma, D, u}^{0}\left(\vec{\rho}_{D} \mid \vec{\rho}_{D^{c}}\right)-F_{\gamma, D, u}^{0}\left(\vec{\rho}_{D}^{*} \mid \vec{\rho}_{D^{c}}\right)=\frac{1}{\beta} \int_{D} d r^{\prime} \sum_{k=1}^{Q} \rho_{k}\left(r^{\prime}\right) \ln \frac{\rho_{k}\left(r^{\prime}\right)}{\rho_{k}^{*}\left(r^{\prime}\right)}
$$

Thus by the Kullback-Leibler inequality, one gets

$$
F_{\gamma, D, u}^{0}\left(\vec{\rho}_{D} \mid \vec{\rho}_{D^{c}}\right)-F_{\gamma, D, u}^{0}\left(\vec{\rho}_{D}^{*} \mid \vec{\rho}_{D^{c}}\right) \geq \frac{1}{2 \beta} \int_{D} d r^{\prime}\left|\vec{\rho}_{D}\left(r^{\prime}\right)-\vec{\rho}_{D}^{*}\left(r^{\prime}\right)\right|^{2}
$$

We claim that there is $\epsilon>0$ such that, for any $\vec{\rho}_{D^{c}}$ and for $\gamma>0$ small enough,

$$
\left\|\vec{\rho}^{*}-\vec{\rho}^{ \pm}\right\|_{\star} \leq(1-\epsilon) \gamma^{a}
$$

Using Cauchy-Schwartz inequality, we thus get (taking $\zeta^{\prime}<\epsilon$ ):

$$
F_{\gamma, D, u}^{0}\left(\vec{\rho}_{D} \mid \vec{\rho}_{D^{c}}\right)-F_{\gamma, D, u}^{0}\left(\vec{\rho}_{D}^{*} \mid \vec{\rho}_{D^{c}}\right) \geq \frac{1}{2 \beta}|D|\left(\epsilon-\zeta^{\prime}\right)^{2} \gamma^{2 a}
$$

Postponing the proof of (7.31), we get the following bound by using proposition 7.6 together with (7.28) and (7.32):

$$
\begin{aligned}
\sup _{\vec{\xi}^{*} \in \mathcal{X}^{( \pm)}} \ln \mu_{\mathrm{abs}, D, u}\left(\mathcal{A}_{x}^{ \pm} \mid \vec{\xi}^{*}\right) & \leq \exp \left\{-\left(\frac{1}{2 \beta}|D|\left(\epsilon-\zeta^{\prime}\right)^{2} \gamma^{2 a}-c^{\prime} \gamma^{d}|D|^{2}\right)+c \gamma^{1 / 2} \ell_{-, d}^{d}\right\} \\
& =\exp \left\{-\left(\frac{1}{2 \beta}\left(\epsilon-\zeta^{\prime}\right)^{2} \gamma^{2 a}-c^{\prime} \gamma^{d \alpha}+c \gamma^{1 / 2}\right) \ell_{-, d}^{d}\right\}
\end{aligned}
$$

The bound (7.24) is then proven for our abstract models. We now turn to the proof of (7.31):

In order to prove (7.31), we show that there is $b \in(0,1)$ :

$$
\left|\rho_{k}^{*}(r)-\rho_{k}^{ \pm}\right| \leq b\left|\mathcal{L}_{k}^{u}(r)-\rho_{k}^{ \pm}\right|
$$

while $\left\|\overrightarrow{\mathcal{L}}^{u}(r)-\vec{\rho}^{ \pm}\right\|_{\star}$ is small and close enough to $\gamma^{a}$.

We first prove that $\left|\mathcal{L}_{k}^{u}(r)-\rho_{k}^{ \pm}\right|$is small enough. We define

$$
J_{\gamma}^{\left(\ell_{-, \gamma}\right)}\left(r,\left\langle r^{\prime}\right\rangle\right):=\frac{1}{\left|C_{r^{\prime}, \gamma}^{\ell_{-}}\right|} \int J_{\gamma}\left(r, r^{\prime \prime}\right) d r^{\prime \prime}
$$

For $\gamma$ small enough, by hypothesis on $\mathcal{J}$ (see (2.2)

$$
\left|J_{\gamma}\left(r, r^{\prime}\right)-J_{\gamma}^{\left(\ell_{-, \gamma}\right)}\left(r,\left\langle r^{\prime}\right\rangle\right)\right| \leq \sqrt{d}\|\nabla \mathcal{J}\|_{\infty} \gamma^{d+1} \ell_{-, \gamma} \mathbf{1}_{\left\{\left|r-r^{\prime}\right| \leq 2 \gamma^{-1}\right\}}
$$


Then, for any $\vec{\rho} \in L^{\infty}\left(\mathbb{R}_{+}^{d}, S_{Q}\right)$, there is a constant $c_{d}=2^{d} \sqrt{d}\|\nabla \mathcal{J}\|_{\infty}$

$$
\left\|J_{\gamma} * \vec{\rho}-J_{\gamma}^{\left(\ell_{-, \gamma}\right)} * \vec{\rho}\right\| \leq c_{d} \gamma \ell_{-, \gamma}=c_{d} \gamma^{\alpha}
$$

Using the fact that $\vec{\rho}_{D^{c}} \in \mathcal{X}_{D^{c}}^{ \pm}$, we write:

$$
\begin{aligned}
\left\|\overrightarrow{\mathcal{L}}^{u}(r)-\vec{\rho}^{ \pm}\right\| & =u\left\|\int_{D^{c}} J_{\gamma}\left(r, r^{\prime}\right) \vec{\rho}_{D^{c}}\left(r^{\prime}\right) d r^{\prime}-\vec{\rho}^{ \pm}\right\| \\
\leq & \left\|\int_{D^{c}}\left(J_{\gamma}\left(r, r^{\prime}\right)-J_{\gamma}^{\left(\ell_{-, \gamma}\right)}\left(r, r^{\prime}\right)\right) \vec{\rho}_{D^{c}}\left(r^{\prime}\right) d r^{\prime}\right\| \\
& \quad+\left\|\int_{D^{c}} J_{\gamma}^{\left(\ell_{-, \gamma}\right)}\left(r, r^{\prime}\right)\left(\vec{\rho}_{D^{c}}\left(r^{\prime}\right)-\vec{\rho}^{ \pm}\right) d r^{\prime}\right\|+\left\|\int_{D} J_{\gamma}^{\left(\ell_{-, \gamma}\right)}\left(r, r^{\prime}\right) \vec{\rho}^{ \pm} d r^{\prime}\right\| \\
\leq & c_{d} \gamma^{\alpha}+\gamma^{a}+c_{d}^{\prime} \gamma^{\alpha d}
\end{aligned}
$$

with $c_{d}^{\prime}=\|\mathcal{J}\|_{\infty}$. Recalling (7.29), we have

$$
\left\|\vec{\rho}_{D}^{*}(r)-\vec{\rho}^{ \pm}\right\|=\left\|\vec{g}\left(\overrightarrow{\mathcal{L}}^{u}(r)\right)-\vec{g}\left(\vec{\rho}^{ \pm}\right)\right\|
$$

Applying Theorem A.3, we get

$$
\begin{aligned}
\left\|\vec{\rho}_{D}^{*}(r)-\vec{\rho}^{ \pm}\right\| & \leq\left(1-\frac{1}{2 Q}\right)\left(\gamma^{a}+c_{d} \gamma^{\alpha}+c_{d}^{\prime} \gamma^{\alpha d}\right) \\
& \leq\left(1-\frac{1}{4 Q}\right) \gamma^{a}-\left(\frac{1}{4 Q} \gamma^{a}-\left(1-\frac{1}{2 Q}\right)\left(c_{d} \gamma^{\alpha}+c_{d}^{\prime} \gamma^{\alpha d}\right)\right) \\
& \leq \gamma^{a}(1-\epsilon)
\end{aligned}
$$

for $\gamma$ small enough and $a<\alpha$, taking $\epsilon=1-\frac{1}{4 Q}$.

\subsubsection{Third requirement.}

Let

$$
\mathcal{B}(n):=\left\{m \in\left(\ell_{-, \gamma} \mathbb{Z}\right)^{d}: \operatorname{dist}\left(C_{n}^{\ell_{-, \gamma}}, C_{m}^{\ell_{-}, \gamma}\right) \leq \gamma^{-1}\right\}
$$

there is $\tilde{r}_{n m}:\left(\ell_{-, \gamma} \mathbb{Z}\right)^{d} \times\left(\ell_{-, \gamma} \mathbb{Z}\right)^{d} \rightarrow \mathbb{R}^{+}$s.t. for any $\vec{\xi}^{1}, \vec{\xi}^{2}: \vec{\xi}^{1}(j)=\vec{\xi}^{2}(j) \forall j \in\left[\mathbb{Z}^{d} \sqcap \mathcal{B}(n)\right] \backslash i$, it holds that:

$$
\begin{aligned}
& R_{C_{n}}\left(\mu_{C}\left(\cdot \mid \vec{\xi}_{C_{n}^{c}}^{(1)}\right), \mu_{C_{n}}\left(\cdot \mid \vec{\xi}_{C_{n}^{c}}^{(2)}\right)\right) \leq \sum_{m \in\left(\ell_{-, \gamma} \mathbb{Z}\right)^{d} \backslash \mathcal{B}(n)} \tilde{r}_{n m} \operatorname{dist}\left(\vec{\xi}_{C_{m}}^{(1)}, \vec{\xi}_{C_{m}}^{(2)}\right) \\
& \sup _{n} \sum_{m \notin \mathcal{B}(n)} \tilde{r}_{n m}<1
\end{aligned}
$$

This is a condition on the tail of the interaction, which in our case is satisfied because of the exponential decay of the interaction $h_{u}^{ \pm}$due to the Peierls estimates. 
7.1.4. Fourth requirement.

Let $r(n, m):\left(\ell_{-, \gamma} \mathbb{Z}\right)^{d} \times\left(\ell_{-, \gamma} \mathbb{Z}\right)^{d} \rightarrow \mathbb{R}^{+}$defined as follows:

$$
r(n, m):= \begin{cases}0 & \text { if } m=n \\ \tilde{r}_{n m} & \text { if } m \notin \mathcal{B}(n) \\ \tilde{r}_{n m}^{*} & \text { if } m \in \mathcal{B}(n) \backslash n\end{cases}
$$

where:

$$
\tilde{r}_{n m}^{*}:=\sup _{j \in C_{m}^{\ell_{-}, \gamma}} \sum_{i \in C_{n}^{\ell_{-}, \gamma}} \sum_{k>0} b_{C_{n}^{\ell_{-}, \gamma}}^{\ell^{-}}(i, j)+2 e^{-c \gamma^{2 a} \ell_{-, \gamma}^{d}}\left(\ell_{-, \gamma}^{d}+\sum_{i, i^{\prime} \in C_{n}^{\ell_{-}, \gamma}} \sum_{k>0} b_{C_{n}^{\ell_{-}, \gamma}}^{k}\left(i, i^{\prime}\right)\right)
$$

with

$$
b_{C_{n}^{\ell_{-, \gamma}}}(i, j):=b(i, j) \mathbf{1}_{i \in C_{n}}
$$

and $b_{C_{n}^{\ell-, \gamma}}^{k}(i, j)$ is the $k$-th convolution of $b_{C_{n}^{\ell_{-}, \gamma}}(i, j)$. Assumption 4 then states:

$$
\sup _{n} \sum_{m} r(n, m) \leq \delta \quad 0<\delta<1
$$

\subsubsection{Fifth Requirement:}

There is a constant $b>0$ such that for all $n \in\left(\ell_{-, \gamma} \mathbb{Z}\right)^{d}$,

$$
\sum_{m \neq n} r(n, m) e^{b \gamma|n-m|}<1
$$

\subsubsection{Conclusion of subsection (7.1).}

We have proved that the two abstract models fulfill the requirements of the extended Dobrusin criterion of reference [3], which imply uniqueness of the measures. Moreover, 5 holds and implies exponential decay of correlations for the measures $\tilde{\mu}_{\mathrm{abs}, u}^{ \pm}$. Hence Theorem 7.3 and Corollary 7.4 are proven.

\subsection{Small deviation estimates.}

In this subsection we prove the estimate (7.13)

$$
\int_{0}^{1} d u \sum_{i \in A}\left\{\left|\left\langle g_{\gamma, i}\right\rangle_{\tilde{\mu}_{\mathrm{abs} ; u}^{ \pm}}\right|+\left|\left\langle g_{\gamma, i, \Lambda}\right\rangle_{\tilde{\mu}_{\mathrm{abs}, \Lambda ; u}^{ \pm}}\right|\right\} \leq c \gamma^{1 / 8}\left|\delta_{\mathrm{in}}^{\ell+, \gamma}[\Lambda]\right|
$$


Let $A:=\delta_{\text {in }}^{\ell+, \gamma}[\Lambda]$ and define the set $S^{ \pm}(\vec{\xi})$ as:

$$
S^{ \pm}(\vec{\xi}):=\left\{i \in \check{A}:\left\|\vec{\xi}^{\left(\ell_{0}\right)}(i)-\vec{\rho}^{ \pm}\right\|_{\star} \geq \gamma^{1 / 8}\right\}
$$

where

$$
\check{A}:=A \sqcup \delta_{\text {out }}^{\gamma^{-1}}[A]
$$

We first prove the following bound:

$$
\begin{aligned}
& \int_{0}^{1} d u \sum_{i \in A}\left\{\left|\left\langle g_{\gamma, i}\right\rangle_{\tilde{\mu}_{\mathrm{abs} ; u}^{ \pm}}\right|+\left|\left\langle g_{\gamma, i, \Lambda}\right\rangle_{\tilde{\mu}_{\mathrm{abs}, \Lambda ; u}^{ \pm}}\right|\right\} \\
& \leq \mathfrak{J}_{\gamma} \int_{0}^{1} d u\left[\left\langle\mathbf{1}_{\left|S^{ \pm}\right| \geq \gamma^{1 / 8}|A|}\right\rangle_{\tilde{\mu}_{\mathrm{abs} ; u}^{ \pm}}+\left\langle\mathbf{1}_{\left|S^{ \pm}\right| \geq \gamma^{1 / 8}|A|}\right\rangle_{\tilde{\mu}_{\mathrm{abs}, \Lambda ; u}^{ \pm}}\right]+c\left(\gamma^{1 / 2}|A|+\gamma^{1 / 8}|A|\right)
\end{aligned}
$$

where

we have denoted by $\mathfrak{J}_{\gamma}$ the normalization constant of the interaction kernel $J_{\gamma}$ on $\mathbb{Z}^{d}$ :

$$
\mathfrak{J}_{\gamma}:=\sum_{j \in \mathbb{Z}^{d}} J_{\gamma}(0, j) \quad \lim _{\gamma \rightarrow 0} \mathfrak{J}_{\gamma}=1
$$

PROOF OF (7.42). We recall the definition of $g_{\gamma, i, \Lambda}(\vec{\xi})$ (7.7) and $g_{\gamma, i}(\vec{\xi})$ (77.8):

$$
\begin{aligned}
g_{\gamma, i, \Lambda}(\vec{\xi}) & :=-\frac{1}{2} \overrightarrow{\delta \xi}_{i} \sum_{\substack{j \in \Lambda \\
j \neq i}} J_{\gamma}(i, j) \overrightarrow{\delta \xi}_{j}+\sum_{\substack{\Delta \ni i \\
\Delta \sqsubset \Lambda}} \frac{1}{|\Delta|} U_{\Delta}^{ \pm}(\vec{\xi}) \\
g_{\gamma, i}(\vec{\xi}) & :=\lim _{\Lambda \nearrow \mathbb{R}^{d}} g_{\gamma, i, \Lambda}(\vec{\xi})=-\frac{1}{2} \overrightarrow{\delta \xi}_{i} \sum_{j \neq i} J_{\gamma}(i, j) \overrightarrow{\delta \xi}_{j}+\sum_{\Delta \ni i} \frac{1}{|\Delta|} U_{\Delta}^{ \pm}(\vec{\xi})
\end{aligned}
$$

By (6.6),

$$
\sum_{\Delta \ni i} \frac{1}{|\Delta|} U_{\Delta}^{ \pm}(\vec{\xi}) \leq e^{-\frac{\xi_{\gamma}}{2}}
$$

Hence, we have:

$$
\begin{aligned}
& \sum_{i \in A}\left|g_{\gamma, i}(\vec{\xi})\right| \leq \frac{1}{2} \sum_{i \in A}\left|\overrightarrow{\delta \xi}_{i} \sum_{j \neq i} J_{\gamma}^{\left(\ell_{0}\right)}(i, j) \overrightarrow{\delta \xi}_{j}\right|+c \gamma \ell_{0}|A| \\
& \leq \sum_{i \in A} \sum_{j \in \check{A}} J_{\gamma}^{\left(\ell_{0}\right)}(i, j)\left\|\overrightarrow{\delta \xi}_{j}^{\left(\ell_{0}\right)}\right\|_{\star}+c \gamma^{1 / 2}|A| \\
& \leq \mathfrak{J}_{\gamma} \sum_{j \in \breve{A}}\left\|\overrightarrow{\delta \xi}_{j}^{\left(\ell_{0}\right)}\right\|_{\star}+c \gamma^{1 / 2}|A| \\
& \leq \mathfrak{J}_{\gamma}\left|S^{ \pm}(\vec{\xi})\right|+c\left(\gamma^{1 / 2}|A|+\gamma^{1 / 8}|A|\right)
\end{aligned}
$$

where we have defined

$$
J_{\gamma}^{\left(\ell_{0}\right)}(i,\langle j\rangle):=\frac{1}{\left|C^{\ell_{0}}\right|} \sum_{j^{\prime} \in C_{j}^{\ell_{0}}} J_{\gamma}(i, j)
$$


and used that by definition (17.40), $\left\|\overrightarrow{\delta \xi_{j}}\right\|_{\star} \leq \gamma^{1 / 8}$ for all $j \notin S$. A similar estimate for $g_{\gamma, i, \Lambda}$ holds and (7.42) follows.

Since the two terms in the integral in the right hand side of (17.42) are very similar, we give the derivation of an estimate for the first term only. We prove the following

$$
\left\langle\mathbf{1}_{\left|S^{ \pm}\right| \geq \gamma^{1 / 8}|A|}\right\rangle_{\tilde{\mu}_{\text {abs } ; u}^{ \pm}} \leq e^{-c \gamma^{3 / 8}|A|}
$$

PROOF OF (7.44).

Let

$$
\begin{gathered}
\hat{A}:=A \sqcup \delta_{\text {out }}^{\ell+, \gamma}[A] \\
\left\langle\mathbf{1}_{\left|S^{ \pm}\right| \geq \gamma^{1 / 8}|A|}\right\rangle_{\tilde{\mu}_{\mathrm{abs} ; u}^{ \pm}}=\left\langle\frac{\hat{\mu}_{\mathrm{abs} ; \hat{A}, u}^{ \pm}\left(\mathbf{1}_{\left|S^{ \pm}\right| \geq \gamma^{1 / 8}|A|} e^{-\beta \sum_{\Delta: \Delta \sqcap \hat{A} \neq \varnothing} U_{\Delta}^{ \pm}(\vec{\xi})} \mid \vec{\xi}_{\hat{A}^{c}}\right)}{\hat{\mu}_{\mathrm{abs} ; \hat{A}, u}^{ \pm}\left(e^{-\beta \sum_{\Delta: \Delta \sqcap \hat{A} \neq \varnothing} U_{\Delta}^{ \pm}(\vec{\xi})} \mid \vec{\xi}_{\hat{A}^{c}}\right)}\right\rangle_{\tilde{\mu}_{\mathrm{abs} ; u}^{ \pm}}
\end{gathered}
$$

where $\hat{\mu}_{\text {abs; } \hat{A}, u}^{ \pm}$is the measure on $\mathcal{X}_{\hat{A}}^{ \pm}$associated to the finite range interpolating Hamiltonian $\hat{h}_{u}^{ \pm}\left(\vec{\xi}_{\hat{A}} \mid \vec{\xi}_{\hat{A}^{c}}\right)$

$$
\hat{h}_{u}^{ \pm}\left(\vec{\xi}_{\hat{A}} \mid \vec{\xi}_{\hat{A}^{c}}\right):=u H_{\gamma, \hat{A}}\left(\vec{\xi}_{\hat{A}} \mid \vec{\xi}_{\hat{A}^{c}}\right)+(1-u) \mathfrak{H}_{\gamma, \hat{A}}^{ \pm}(\vec{\xi})
$$

Recalling (6.6), we get:

$$
\left\langle\mathbf{1}_{\left|S^{ \pm}\right| \geq \gamma^{1 / 8}|A|}\right\rangle_{\tilde{\mu}_{\mathrm{abs} ; u}^{ \pm}} \leq\left\langle\hat{\mu}_{\mathrm{abs} ; \hat{A}, u}^{ \pm}\left(\mathbf{1}_{\left|S^{ \pm}\right| \geq \gamma^{1 / 8}|A|} \mid \vec{\xi}_{\hat{A}^{c}}\right)\right\rangle_{\tilde{\mu}_{\mathrm{abs} ; u}^{ \pm}} \times e^{\beta|\hat{A}| e^{-\frac{\mathfrak{k}_{\gamma}}{2}}}
$$

we write

$$
\hat{\mu}_{\mathrm{abs} ; \hat{A}, u}^{ \pm}\left(\mathbf{1}_{\left|S^{ \pm}\right| \geq \gamma^{1 / 8}|A|} \mid \vec{\xi}_{\hat{A}^{c}}\right)=\frac{\hat{Z}_{\mathrm{abs}, \beta, \hat{A} ; u}^{ \pm}\left(S^{ \pm} \mid \vec{\xi}_{\hat{A}^{c}}\right)}{\hat{Z}_{\mathrm{abs}, \beta, \hat{A} ; u}^{ \pm}\left(\vec{\xi}_{\hat{A}^{c}}\right)}
$$

where

$$
\begin{aligned}
\hat{Z}_{\mathrm{abs}, \beta, \hat{A} ; u}^{ \pm}\left(S^{ \pm} \mid \vec{\xi}_{\hat{A}^{c}}\right):= & \sum_{\vec{\xi}_{\hat{A}} \in \mathcal{X}_{\hat{A}}^{ \pm}} \mathbf{1}_{\left|S^{ \pm}\right| \geq \gamma^{1 / 8}|A|} e^{-\beta \hat{h}_{u}^{ \pm}\left(\vec{\xi}_{\hat{A}^{\prime}} \mid \vec{\xi}_{\hat{A}^{c}}\right)} \\
\hat{Z}_{\mathrm{abs}, \beta, \hat{A} ; u}^{ \pm}\left(\vec{\xi}_{\hat{A}^{c}}\right):= & \sum_{\vec{\xi}_{\hat{A}} \in \mathcal{X}_{\hat{A}}^{ \pm}} e^{-\beta \hat{h}_{u}^{ \pm}\left(\vec{\xi}_{\hat{A}} \mid \vec{\xi}_{\hat{A}^{c}}\right)}
\end{aligned}
$$

Now the partition function $\hat{Z}_{\text {abs, } \beta, \hat{A} ; u}^{ \pm}\left(S^{ \pm} \mid \vec{\xi}_{\hat{A}^{c}}\right)$ can be estimated using an approximation to the continuum. Defining

$$
\begin{aligned}
F_{\gamma, \hat{A}, u}\left(\vec{\xi}_{\hat{A}} \mid \vec{\xi}_{\hat{A}^{c}}^{\left(\ell_{0}\right)}\right):= & -\frac{1}{2} \int_{\hat{A} \times \hat{A}} J_{\gamma}\left(r, r^{\prime}\right)\left(u \vec{\xi}_{\hat{A}}(r) \cdot \vec{\xi}_{\hat{A}}\left(r^{\prime}\right)+(1-u) \vec{\rho}^{ \pm} \cdot \vec{\rho}^{ \pm}\right) d r d r^{\prime} \\
& -\int_{\hat{A} \times \hat{A}^{c}} J_{\gamma}\left(r, r^{\prime}\right)\left(u \vec{\xi}_{\hat{A}}(r) \cdot \vec{\xi}_{\hat{A}^{c}}^{\left(\ell_{0}\right)}\left(r^{\prime}\right)+(1-u) \vec{\rho}^{ \pm} \cdot \vec{\rho}^{ \pm}\right) d r d r^{\prime} \\
& -(1-u) \int_{\hat{A}}\left(\vec{\xi}_{\hat{A}}(r) \cdot \vec{\rho}^{ \pm}\right) d r+\frac{1}{\beta} \int_{\hat{A}} \vec{\xi}_{\hat{A}}(r) \cdot \ln \vec{\xi}_{\hat{A}}(r) d r
\end{aligned}
$$


and

$\mathfrak{Z}^{ \pm}:=\left\{\vec{\xi}_{\hat{A}} \in \Omega_{\Lambda}^{\ell_{0}}: \vec{\xi}_{\hat{A}^{\prime}}(r)=\vec{\xi}^{\left(\ell_{0}\right)}(r) ; \eta(\vec{\xi} ; r)=a_{ \pm} r \in \hat{A} ; \int_{\breve{A}} \mathbf{1}_{\left\|\vec{\xi}_{\hat{A}^{-}} \vec{\rho}^{ \pm}\right\|_{*} \geq \gamma^{1 / 8}} \geq \gamma^{1 / 8}|A|\right\}$

A result similar to theorem 5.1 holds for the above functional and leads to

$$
\ln \hat{Z}_{\text {abs, }, \beta, \hat{A} ; u}^{ \pm}\left(S^{ \pm} \mid \vec{\xi}_{\hat{A}^{c}}\right) \leq-\beta \inf _{\vec{\xi}_{\hat{A}} \in \mathfrak{Z}^{ \pm}} F_{\gamma, \hat{A}, u}\left(\vec{\xi}_{\hat{A}} \mid \vec{\xi}_{\hat{A}^{c}}^{\left(\ell_{0}\right)}\right)+c \gamma^{1 / 2}|\hat{A}|
$$

where $c$ is a constant independent on $u$.

We are then reduced to study the variational problem in (17.48) for $F_{\gamma, \hat{A}, u}\left(\vec{\xi}_{\hat{A}} \mid \vec{\xi}_{\hat{A}^{c}}^{\left(\ell_{0}\right)}\right)$ on $\mathfrak{Z}^{ \pm}$. We define the "excess free energy functional":

$$
\begin{gathered}
\mathcal{F}_{\gamma, \hat{A}, u}^{\mathrm{eff}}\left(\vec{\xi}_{\hat{A}} \mid \vec{\xi}_{\hat{A}^{c}}^{\left(\ell_{0}\right)}\right):=F_{\gamma, \hat{A}, u}\left(\vec{\xi}_{\hat{A}} \mid \vec{\xi}_{\hat{A}^{c}}^{\left(\ell_{0}\right)}\right)+\mathfrak{A} \\
\mathfrak{A}:=-\inf _{\vec{v}:\|\vec{v}\|=1}^{\mathrm{mf}}(\vec{v})|\hat{A}|+\frac{u}{2} \int_{\hat{A} \times \hat{A}^{c}} J_{\gamma}\left(r, r^{\prime}\right)\left(\vec{\xi}_{\hat{A}^{c}}^{\left(\ell_{0}\right)}\left(r^{\prime}\right) \cdot \vec{\xi}_{\hat{A}^{c}}^{\left(\ell_{0}\right)}\left(r^{\prime}\right)\right) d r d r^{\prime} \\
\phi_{u}^{\mathrm{mf}, \pm}(\vec{v}):=-u \frac{(\vec{v} \cdot \vec{v})}{2}-(1-u)\left(\vec{v} \cdot \rho^{ \pm}\right)+\frac{1}{\beta}(\vec{v} \cdot \ln \vec{v})
\end{gathered}
$$

$\mathcal{F}_{\gamma, \hat{A}, u}^{\text {eff }}\left(\vec{\xi}_{\hat{A}} \mid \vec{\xi}_{\hat{A}^{c}}^{\left(\ell_{0}\right)}\right)$ is positive and differs from $F_{\gamma, \hat{A}, u}\left(\vec{\xi}_{\hat{A}} \mid \vec{\xi}_{\hat{A}^{c}}^{\left(\ell_{0}\right)}\right)$ by a constant and hence has the same minimizers and its minimum is finite

Denoting by:

$$
\Phi_{u}^{\mathrm{eff}, \pm}\left(\vec{\xi}_{\hat{A}}\right):=\phi_{u}^{\mathrm{mf}, \pm}\left(\vec{\xi}_{\hat{A}}\right)-\inf _{\vec{v}:\|\vec{v}\|=1} \phi_{u}^{\mathrm{mf}, \pm}(\vec{v})
$$

$\mathcal{F}_{\gamma, u}^{\mathrm{eff}}\left(\vec{\xi}_{\hat{A}} \mid \vec{\xi}_{\hat{A}^{c}}^{\left(\ell_{0}\right)}\right)$ can be rewritten then as:

$$
\begin{aligned}
\mathcal{F}_{\gamma, \hat{A}, u}^{\mathrm{eff}}\left(\vec{\xi}_{\hat{A}} \mid \vec{\xi}_{\hat{A}^{c}}^{\left(\ell_{0}\right)}\right)=\int_{\hat{A}} \Phi_{u}^{\mathrm{eff}, \pm}\left(\vec{\xi}_{\hat{A}}\right)+\frac{u}{4} \int_{\hat{A} \times \mathbb{B}} J_{\gamma}\left(r, r^{\prime}\right)\left[\vec{\xi}_{\hat{A}}(r)-\vec{\xi}_{\hat{A}^{\prime}}\left(r^{\prime}\right)\right]^{2} & \\
& +\frac{u}{2} \int_{\hat{A} \times \hat{A}^{c}} J_{\gamma}\left(r, r^{\prime}\right)\left[\vec{\xi}_{\hat{A}}(r)-\vec{\xi}_{\hat{A}^{c}}^{\left(\ell_{0}\right)}\left(r^{\prime}\right)\right]^{2}
\end{aligned}
$$

The analysis in appendix B, see corollary B.5 proves that there are positive constants $\omega, c_{\omega}$, so that for any $\vec{\xi} \in \mathfrak{Z}^{ \pm}$there is $\vec{\psi}_{\hat{A}}$ :

$$
\mathcal{F}_{\gamma, \hat{A}, u}^{\mathrm{eff}}\left(\vec{\xi}_{\hat{A}} \mid \vec{\xi}_{\hat{A}^{c}}^{\left(\ell_{0}\right)}\right) \geq \mathcal{F}_{\gamma, \hat{A}, u}^{\mathrm{eff}}\left(\vec{\psi}_{\hat{A}} \mid \vec{\xi}_{\hat{A}^{c}}^{\left(\ell_{0}\right)}\right)-c_{\omega} e^{-\omega \gamma \ell_{+, \gamma} / 4}|\hat{A}|
$$

where $\vec{\Psi}_{\hat{A}}$ has the following properties:

$$
\begin{aligned}
& \eta\left(\vec{\psi}_{\hat{A}} ; r\right)=a_{ \pm} \quad \forall r \in \hat{A} \\
& \vec{\psi}_{\hat{A}}(r)=\vec{\rho}^{ \pm} \quad \forall r \in \Sigma:=(\hat{A} \backslash A) \backslash \delta_{\text {in }}^{\ell_{+}, r} / 4(\hat{A} \backslash A) \\
& \vec{\psi}_{\hat{A}}(r)=\vec{\xi}(r) \quad \forall r \in \check{A} \\
& \sup _{q}\left|\psi_{\hat{A}, q}(r)-\rho_{q}^{ \pm}\right| \leq\left(1-\kappa_{0}\right) \gamma^{a} \quad \forall r \in \delta_{\text {in }}^{\ell_{+}, \gamma} / 4[\hat{A}] \quad \kappa_{0}>0
\end{aligned}
$$

We can write: 


$$
\ln \hat{Z}_{\beta, \hat{A} ; u}^{ \pm}\left(S^{ \pm} \mid \vec{\xi}_{\hat{A}^{c}}\right) \leq-\beta \inf _{\vec{\psi}_{\hat{A}} \in \mathcal{B}^{0}} \mathcal{F}_{\gamma, \hat{A}, u}^{\mathrm{eff}}\left(\vec{\psi}_{\hat{A}^{\prime}} \mid \vec{\xi}_{\hat{A}^{c}}^{\left(\ell_{0}\right)}\right)+c \gamma \ell_{0}|\hat{A}|+\beta c_{\omega} e^{-\omega \gamma \ell_{+, \gamma} / 4}|\hat{A}|+\mathfrak{A}
$$

where

$$
\begin{aligned}
\mathcal{B}^{0}:= & \left\{\vec{\psi}_{\hat{A}}: \sup _{q}\left|\psi_{\hat{A}, q}-\rho_{q}^{ \pm}\right| \leq\left(1-\kappa_{0}\right) \gamma^{a}, r \in \delta_{\text {in }}^{\ell_{+}, \gamma / 4}[\hat{A}] ; \quad \eta\left(\vec{\psi}_{\hat{A}} ; r\right)=a_{ \pm}, r \in \hat{A} ;\right. \\
& \left.\vec{\psi}_{\hat{A}}=\vec{\rho}^{ \pm} r \in \Sigma ; \quad \vec{\psi}_{\hat{A}}(r)=\vec{\psi}_{\hat{A}}^{\left(\ell_{0}\right)}(r), r \in \check{A} ; \quad \int_{\check{A}} \mathbf{1}_{\left\|\vec{\psi}_{\hat{A}, q}-\vec{\rho}^{ \pm}\right\|_{\star}} \geq \gamma^{1 / 8}>\gamma^{1 / 8}|A|\right\}
\end{aligned}
$$

In appendix $\mathrm{E}$ it is proved that for any $\psi_{\hat{A}} \in \mathcal{B}^{0}$ there is $\vec{\psi}_{\hat{A}}^{*}$ :

$$
\vec{\psi}_{\hat{A}}^{*}= \begin{cases}\vec{\psi}_{\hat{A}} & \text { on } \delta_{\text {in }}^{\ell_{+} / 4}[\hat{A}] \\ \vec{\rho}^{ \pm} & \text {elsewhere. }\end{cases}
$$

so that:

$$
\mathcal{F}_{\gamma, \hat{A}, u}^{\mathrm{eff}}\left(\vec{\psi}_{\hat{A}} \mid \vec{\xi}_{\hat{A}^{c}}^{\left(\ell_{0}\right)}\right) \geq \mathcal{F}_{\gamma, \hat{A}, u}^{\mathrm{eff}}\left(\vec{\psi}_{\hat{A}}^{*} \mid \vec{\xi}_{\hat{A}^{c}}^{\left(\ell_{0}\right)}\right)+c \gamma^{1 / 4}\left(\gamma^{1 / 8}|A|\right)
$$

Let $\left[\psi^{*}\right]^{\ell_{0}}$ defined as in (5.28). By theorem 5.1 we then have:

$$
-\beta F_{\gamma, \hat{A}, u}\left(\vec{\psi}_{\hat{A}}^{*} \mid \vec{\xi}_{\hat{A}^{c}}^{\left(\ell_{0}\right)}\right) \leq \ln \hat{Z}_{\beta, \hat{A} ; u}\left(\left\{\vec{\xi}_{\hat{A}}^{\ell_{0}}=\left[\vec{\psi}_{\hat{A}}^{*}\right]^{\ell_{0}}\right\} \mid \vec{\xi}_{\hat{A}^{c}}\right)+\gamma \ell_{0}|\hat{A}|
$$

and since by definition :

$$
\left\|\left[\vec{\psi}_{\hat{A}}^{*}\right]^{\ell_{0}}-\vec{\psi}_{\hat{A}}^{*}\right\|_{\star}<\frac{1}{2} \ell_{0}^{-d}
$$

we have:

$$
\left\|\left[\vec{\psi}_{\hat{A}}^{*}\right]_{q}^{\ell_{0}}-\vec{\rho}^{ \pm}\right\|_{\star}<\frac{1}{2} \ell_{0}^{-d}+\left(1-\kappa_{0}\right) \gamma^{a}
$$

so that the set $\left\{\vec{\xi}_{\hat{A}}^{\ell_{0}}=\left[\vec{\psi}_{\hat{A}}^{*}\right]^{\ell_{0}}\right\} \in \mathcal{X}^{ \pm}$and

$$
-\beta F_{\gamma, \hat{A}, u}\left(\vec{\psi}_{\hat{A}}^{*} \mid \vec{\xi}_{\hat{A}^{c}}^{\left(\ell_{0}\right)}\right) \leq \ln \hat{Z}_{\text {abs }, \beta, \hat{A} ; u}^{ \pm}\left(\vec{\xi}_{\hat{A}^{c}}\right)+\gamma \ell_{0}|\hat{A}|
$$

By (7.49) and (7.55)

$\ln \hat{Z}_{\text {abs, } \beta, \hat{A} ; u}^{ \pm}\left(S^{ \pm} \mid \vec{\xi}_{\hat{A}^{c}}\right) \leq \ln \hat{Z}_{\text {abs }, \beta, \hat{A} ; u}^{ \pm}\left(\vec{\xi}_{\hat{A}^{c}}\right)+\beta c_{\omega} e^{-\omega \gamma \ell_{+, \gamma} / 4}|\hat{A}|-c \gamma^{1 / 4}\left(\gamma^{1 / 8}|A|\right)+c^{\prime} \gamma \ell_{0}|\hat{A}|$ Inserting this inequality in (7.45), we get (7.44) for $\gamma$ small enough. 


\section{Factorization theorem and large deviation estimate}

In this section we prove the factorization theorem 4.2 in a slightly different form, proving at once factorization and control through the mean field functional, for which we prove the large deviation estimate needed to get the Peierls bound of Theorem 4.3

Let $\Gamma$ a $\hat{p}$-contour and define

$$
G^{\hat{p}}:=\operatorname{sp}(\Gamma) \bigsqcup_{\hat{q} \neq \hat{p}} A^{\hat{q}}
$$

Theorem 8.1. There are $\bar{\gamma}, \bar{b}$ and a constant $c$ such that for all $\gamma<\bar{\gamma},\left|\beta-\beta_{c}^{\operatorname{mf}}\right|<\bar{b}$ :

$$
\begin{aligned}
& w_{\gamma, \beta}^{\hat{p}}\left(\Gamma ; \vec{\xi}_{A^{\hat{p}}}\right) \leq \frac{\prod_{\hat{q} \neq \hat{p}} Z_{\gamma, \beta, \operatorname{Int}^{q}(\Gamma) \backslash A^{\hat{q}}}^{\hat{q}}\left(\hat{\rho}^{\hat{q}}\right)}{\prod_{\hat{q} \neq \hat{p}} Z_{\gamma, \beta, \operatorname{Int}^{\hat{q}}(\Gamma) \backslash A^{\hat{q}}}^{\hat{p}}\left(\vec{\rho}^{\hat{p}}\right)} \quad \exp \left\{c \gamma^{\frac{1}{2}}|\Gamma|\right\} \\
& \cdot \exp \left\{-\beta\left[\inf _{\substack{\vec{\rho}_{G \hat{p}}: \eta\left(\vec{\rho}_{G \hat{p}}\right)=\eta_{\Gamma} \\
\vec{\rho}_{A^{\hat{q}}=\vec{\rho}}{ }^{\underline{q}}}} F_{\gamma, \beta, G \hat{p}}\left(\vec{\rho}_{G^{\hat{p}}} \mid \vec{\xi}_{A^{\hat{p}}}^{\left(\ell_{0}\right)}\right)-\inf _{\substack{\vec{\rho}_{G \hat{p}}: \eta\left(\vec{\rho}_{G \hat{p}}\right)=a^{p} \\
\vec{\rho}_{A^{\hat{q}}}=\vec{\rho} \hat{p}}} F_{\gamma, \beta, G \hat{p}}\left(\vec{\rho}_{G^{\hat{p}}} \mid \vec{\xi}_{A^{p}}^{\left(\ell_{0}\right)}\right)\right]\right\}
\end{aligned}
$$

In order to short notations we define $\mathcal{R}(\Gamma)$ and $\mathcal{R}(X)$ as the two subsets of $L^{\infty}\left(G^{\hat{p}}, S_{Q}\right)$ such that

$$
\begin{gathered}
\mathcal{R}(\Gamma):=\left\{\vec{\rho} \in L^{\infty}\left(G^{\hat{p}}, S_{Q}\right): \eta_{x}(\vec{\rho})=\eta_{\Gamma}(x) \forall x \in \operatorname{sp}(\Gamma) ; \quad \vec{\rho}(x)=\vec{\rho}^{\hat{q}} \forall x \in A^{\hat{q}} \forall \hat{q} \neq \hat{p}\right\} \\
\mathcal{R}(\not \Gamma):=\left\{\vec{\rho} \in L^{\infty}\left(G^{\hat{p}}, S_{Q}\right): \eta(\vec{\rho}, x)=a_{\hat{p}} \forall x \in \Gamma ; \quad \rho(x)=\vec{\rho}^{\hat{p}} \forall x \in \bigsqcup_{\hat{q} \neq \hat{p}} A^{\hat{q}}\right\}
\end{gathered}
$$

We will prove also the following Theorem:

Theorem 8.2. There exists $\bar{\gamma}>0$ and a constant $c_{f}>0$ such that for all $\gamma \leq \bar{\gamma}$ and all $\beta$ such that $\left|\beta-\beta_{c}^{\mathrm{mf}}\right| \leq c_{b} \gamma^{\frac{1}{2}}$, the following large deviation estimate holds:

$$
\inf _{\vec{\rho} \in \mathcal{R}(\Gamma)} F_{\gamma, \beta, G \hat{p}}\left(\vec{\rho} \mid \vec{\xi}_{A^{\hat{p}}}^{\left(\ell_{0}\right)}\right)-\inf _{\vec{\rho} \in \mathcal{R}(\not))} F_{\gamma, \beta, G \hat{p}}\left(\vec{\rho} \mid \vec{\xi}_{A^{\hat{p}}}^{\left(\ell_{0}\right)}\right) \geq c_{f}|\Gamma| \gamma^{2 a}\left(\frac{\ell_{-, \gamma}}{\ell_{+, \gamma}}\right)^{d}+\sum_{\hat{q} \neq \hat{p}} \mathcal{I}_{A^{\hat{q}}}\left(\vec{\rho}_{\hat{q}}^{2}-\vec{\rho}_{\hat{p}}^{2}\right)
$$

where

$$
\mathcal{I}_{A^{\hat{q}}}=\frac{1}{2} \int_{A^{\hat{q}}} \int_{\left(\operatorname{Int}^{\hat{q}}(\Gamma) \backslash A^{\hat{q}}\right)} J_{\gamma}(x, y) d x d y
$$


We first recall the definition of the weight of a $\hat{p}$-contour given in (3.17):

$$
w_{\gamma, \beta}^{\hat{p}}\left(\Gamma ; \vec{\xi}_{A \hat{p}}\right):=\frac{\mu_{\gamma, \beta, c(\Gamma) \backslash \operatorname{Int}^{\hat{p}}(\Gamma)}\left(\mathcal{E}(\Gamma, \hat{p}) \mid \vec{\xi}_{A \hat{p}}\right)}{\mu_{\gamma, \beta, c(\Gamma) \backslash \operatorname{Int}^{\hat{p}}(\Gamma)}\left(\mathcal{E}(\not, \hat{p}) \mid \vec{\xi}_{A^{\hat{p}}}\right)}
$$

For each set $A^{\hat{q}}, \hat{q} \neq \hat{p}$, we denote by:

$$
\begin{aligned}
\hat{A}^{\hat{q}} & :=A^{\hat{q}} \sqcup \delta_{\text {out }}^{\ell+, \gamma}\left[A^{\hat{q}}\right] \\
\check{A}^{\hat{q}} & :=A^{\hat{q}} \sqcup \delta_{\text {out }}^{\ell+, \gamma} / 2\left[A^{\hat{q}}\right] \\
\Sigma^{\hat{q}, e} & :=\left(\hat{A}^{\hat{q}} \backslash \check{A}^{\hat{q}}\right) \sqcap \operatorname{sp}(\Gamma)^{c} \\
\bar{\Sigma}^{\hat{q}, e} & :=\left(\check{A}^{\hat{q}} \backslash A^{\hat{q}}\right) \sqcap \operatorname{sp}(\Gamma)^{c} \\
\Sigma^{e} & :=\sqcup_{\hat{q} \neq \hat{p}} \Sigma^{\hat{q}, e}
\end{aligned}
$$

and also:

$$
\begin{aligned}
\Delta^{\hat{p}} & :=\operatorname{sp}(\Gamma) \sqcup_{\hat{q} \neq \hat{p}} \check{A}^{\hat{q}} \\
V^{\hat{q}} & :=\operatorname{Int}^{\hat{q}}(\Gamma) \backslash \hat{A}^{\hat{q}}
\end{aligned}
$$

We write:

$$
w_{\gamma, \beta}^{\hat{p}}\left(\Gamma ; \vec{\xi}_{A^{\hat{p}}}\right):=\frac{\sum_{\vec{\xi}_{\Sigma e}: \vec{\xi}_{\Sigma^{\hat{q}, e}} \in \mathcal{X}^{\hat{q}}} e^{-\beta \sum_{\hat{q} \neq \hat{p}} H_{\gamma}\left(\vec{\xi}_{\Sigma^{\hat{q}, e}}\right)} Z_{\beta, \Delta \hat{p}}\left(\mathcal{E}(\Gamma) \mid \vec{\xi}_{\Sigma e} ; \vec{\xi}_{A^{\hat{p}}}\right) \prod_{\hat{q} \neq \hat{p}} Z_{\beta, V^{\hat{q}}}^{\hat{q}}\left(\vec{\xi}_{\Sigma^{\hat{q}, e}}\right)}{\sum_{\vec{\xi}_{\Sigma^{e}} \in \mathcal{X}^{\hat{p}}} e^{-\beta H_{\gamma}\left(\vec{\xi}_{\Sigma^{e}}\right)} Z_{\beta, \Delta \hat{p}}\left(\mathcal{E}(X) \mid \vec{\xi}_{\Sigma^{e}} ; \vec{\xi}_{A^{\hat{p}}}\right) \prod_{\hat{q} \neq \hat{p}} Z_{\beta, V^{\hat{q}}}^{\hat{p}}\left(\vec{\xi}_{\Sigma^{\hat{q}, e}}\right)}
$$

By Theorem 5.1 with $\ell=\ell_{0}$, we have:

$$
\begin{aligned}
& w_{\gamma, \beta}^{\hat{p}}\left(\Gamma ; \vec{\xi}_{A \hat{p}}\right) \leq e^{c \gamma \ell_{0}|\Gamma|} .
\end{aligned}
$$

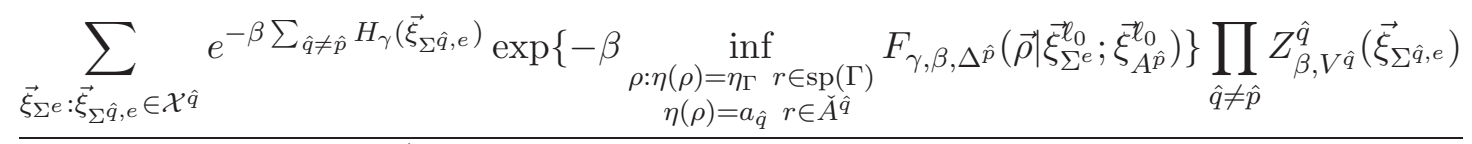

$$
\begin{aligned}
& \sum_{\vec{\xi}_{\Sigma^{e}} \in \mathcal{X} \hat{p}} e^{-\beta H_{\gamma}\left(\vec{\xi}_{\Sigma^{e}}\right)} \exp \left\{-\beta \inf _{\rho: \eta(\rho)=a_{\hat{p}}} F_{\gamma, \beta, \Delta \hat{p}}\left(\vec{\rho} \mid \vec{\xi}_{\Sigma^{e}}^{\ell_{0}} ; \vec{\xi}_{A^{\hat{p}}}^{\ell_{0}}\right)\right\} \prod_{\hat{q} \neq \hat{p}} Z_{\beta, V^{\hat{q}}}^{\hat{p}}\left(\vec{\xi}_{\Sigma^{\hat{q}, e}}\right)
\end{aligned}
$$

Using Corollary B.5 with $\Lambda=\check{A}^{\hat{q}}$, we can find a lower bound for the free energy term in the numerator by considering density profiles $\vec{\rho}$ identically equal to $\vec{\rho}^{\hat{q}}$ on $A^{\hat{q}}$, at the expense of a small error term; we have:

$$
\begin{aligned}
& \inf _{\substack{\rho: \eta(\rho)=\eta_{\Gamma} \\
\eta(\rho)=a_{\hat{q}}}} F_{\gamma \in \tilde{A}^{\hat{q}}} F_{\gamma, \beta, \Delta \hat{p}}\left(\vec{\rho} \mid \vec{\xi}_{\Sigma^{e}}^{\ell_{0}} ; \vec{\xi}_{A^{\hat{p}}}^{\ell_{0}}\right) \geq \inf _{\substack{\rho: \eta(\rho)=\eta_{\Gamma} r \in \operatorname{sp}(\Gamma) \\
\eta(\rho)=a_{\hat{q}} r \in \tilde{A}^{\hat{q}} \\
\vec{\rho} \equiv \vec{\rho}^{\hat{q}} r \in A^{\hat{q}}}} F_{\gamma, \beta, \Delta \hat{p}}\left(\vec{\rho} \mid \vec{\xi}_{\Sigma^{e}}^{\ell_{0}} ; \vec{\xi}_{A^{\hat{p}}}^{\ell_{0}}\right)-c_{\omega} e^{-\omega \gamma \ell_{+, \gamma} / 2}|\check{A}| \\
& =\inf _{\substack{\rho: \eta(\rho)=\eta_{\Gamma} \\
\vec{\rho} \equiv \vec{\rho}^{\hat{q}} r \in A^{\hat{q}}}} F_{\gamma, \beta, G)}\left(\vec{\rho} \mid \vec{\xi}_{\Sigma^{e}}^{\ell_{0}} ; \vec{\xi}_{A^{\hat{p}}}\right)+\sum_{\hat{q}} \inf _{\rho: \eta(\rho)=a_{\hat{q}}} F_{\gamma, \beta, \bar{\Sigma} \hat{q}, e}\left(\vec{\rho} \mid \vec{\xi}_{\Sigma^{e}}^{\ell_{0}} ; \vec{\rho}_{A^{\hat{q}}}^{q}\right)-c_{\omega} e^{-\omega \gamma \ell_{+, \gamma} / 2}|\Gamma| \\
& \inf _{\substack{\rho: \eta(\rho)=\eta_{\Gamma} r \in \operatorname{sp}(\Gamma) \\
\vec{\rho} \equiv \overline{\rho^{\hat{q}}} r \in A^{\hat{q}}}} F_{\gamma, \beta, G^{\hat{p}}}\left(\vec{\rho} \mid \vec{\xi}_{\Sigma^{e}}^{\ell_{0}} ; \vec{\xi}_{A^{\hat{p}}}\right)-\frac{1}{\beta} \sum_{\hat{q}} \log \left(Z_{\beta, \bar{\Sigma} \hat{q}, e}\left(\vec{\xi}_{\Sigma^{e}} ; \vec{\rho}_{A^{\hat{q}}}^{q}\right)\right)-\left(c_{\omega} e^{-\omega \gamma \ell_{+, \gamma} / 2}+c \gamma \ell_{0}\right)|\Gamma|
\end{aligned}
$$


The free energy term in the denominator of (8.8) can be directly bounded from above, as

$$
\begin{aligned}
& \inf _{\rho: \eta(\rho)=a_{\hat{p}}} F_{\gamma, \beta, \Delta \hat{p}}\left(\vec{\rho} \mid \vec{\xi}_{\Sigma^{e}}^{\ell_{0}} ; \vec{\xi}_{A^{\hat{p}}}^{\ell_{0}}\right) \leq \inf _{\substack{\rho: \eta(\rho)=a_{\hat{p}} \\
\vec{\rho} \equiv \bar{\rho}^{\hat{q}} r \in A^{\hat{q}}}} F_{\gamma, \beta, \Delta \hat{p}}\left(\vec{\rho} \mid \vec{\xi}_{\Sigma^{e}}^{\ell_{0}} ; \vec{\xi}_{A^{\hat{p}}}^{\ell_{0}}\right) \\
& =\inf _{\rho: \eta(\rho)=a_{\hat{p}}} F_{\gamma, \beta, G^{\hat{p}}}\left(\vec{\rho} \mid \vec{\xi}_{\Sigma^{e}}^{\ell_{0}} ; \vec{\xi}_{A^{\hat{p}}}\right)+\sum_{\hat{q}} \inf _{\rho: \eta(\rho)=a_{\hat{p}}} F_{\gamma, \beta, \bar{\Sigma} \hat{q}, e}\left(\vec{\rho} \mid \vec{\xi}_{\Sigma^{e}}^{\ell_{0}} ; \vec{\rho}_{A^{\hat{q}}}^{\hat{p}}\right) \\
& \leq \inf _{\rho: \eta(\rho)=a_{\hat{p}}} F_{\gamma, \beta, G^{\hat{p}}}\left(\vec{\rho} \mid \vec{\xi}_{\Sigma^{e}}^{\ell_{0}} ; \vec{\xi}_{A^{\hat{p}}}\right)-\frac{1}{\beta} \sum_{\hat{q}} \log \left(Z_{\beta, \overline{\Sigma^{\hat{q}}, e}}\left(\vec{\xi}_{\Sigma^{e}} ; \vec{\rho}_{A^{\hat{q}}}^{\hat{p}}\right)\right)+c \gamma \ell_{0}|\Gamma|
\end{aligned}
$$

Inserting both estimates in (8.8) we get the following factorization:

$$
\begin{aligned}
& w_{\gamma, \beta}^{\hat{p}}\left(\Gamma ; \vec{\xi}_{A \hat{p}}\right) \\
& \leq e^{\beta\left(3 c \gamma \ell_{0}+c_{\omega} e^{-\omega \gamma \ell_{+, \gamma} / 2}\right)|\Gamma|} \frac{\sum_{\vec{\xi}_{\Sigma^{e}}: \vec{\xi}_{\Sigma^{\hat{q}, e}} \in \mathcal{X} \hat{q}} \prod_{\hat{q} \neq \hat{p}} e^{-\beta H_{\gamma}\left(\vec{\xi}_{\Sigma^{\hat{q}, e}}\right)} Z_{\beta, \bar{\Sigma} \hat{q}, e}\left(\vec{\xi}_{\Sigma^{e}} ; \vec{\rho}_{A^{\hat{q}}}^{q}\right) Z_{\beta, V^{\hat{q}}}^{\hat{q}}\left(\vec{\xi}_{\Sigma^{\hat{q}, e}}\right)}{\sum_{\vec{\xi}_{\Sigma^{e}} \in \mathcal{X} \hat{p}} \prod_{\hat{q} \neq \hat{p}} e^{-\beta H_{\gamma}\left(\vec{\xi}_{\Sigma^{\hat{q}, e}}\right)} Z_{\beta, \bar{\Sigma} \hat{q}, e}\left(\vec{\xi}_{\Sigma^{e}} ; \vec{\rho}_{A^{\hat{q}}}^{\hat{p}}\right) Z_{\beta, V^{\hat{q}}}^{\hat{p}}\left(\vec{\xi}_{\Sigma^{\hat{q}, e}}\right)} \\
& \times \exp \left\{-\beta\left(\inf _{\substack{\rho: \eta(\rho)=\eta_{\Gamma} \\
\vec{\rho} \equiv \bar{\rho}^{\hat{q}} r \in A^{\hat{q}}}} F_{\gamma, \beta, G^{\hat{p}}}\left(\vec{\rho} \mid \vec{\xi}_{\Sigma^{e}}^{\ell_{0}} ; \vec{\xi}_{A^{\hat{p}}}\right)-\inf _{\rho: \eta(\rho)=a^{p}} F_{\gamma, \beta, G \hat{p}}\left(\vec{\rho} \mid \vec{\xi}_{\Sigma^{e}}^{\ell_{0}} ; \vec{\xi}_{A^{\hat{p}}}\right)\right)\right\} \\
& =e^{3 \beta c \gamma \ell_{0}|\Gamma|+\beta c_{\omega} e^{-\omega \gamma \ell_{+, \gamma} / 2}} \frac{\prod_{\hat{q} \neq \hat{p}} Z_{\gamma, \beta, \operatorname{Int}^{q}(\Gamma) \backslash A^{\hat{q}}}^{\hat{q}}\left(\vec{\rho}^{\hat{q}}\right)}{\prod_{\hat{q} \neq \hat{p}} Z_{\gamma, \beta, \operatorname{Int}^{\hat{q}}(\Gamma) \backslash A^{\hat{q}}}^{\hat{p}}\left(\vec{\rho}^{\hat{p}}\right)} \\
& \cdot \exp \left\{-\beta\left[\inf _{\substack{\vec{\rho}_{G \hat{p}}: \eta\left(\vec{\rho}_{G \hat{p}}\right)=\eta_{\Gamma} \\
\vec{\rho}_{A \hat{q}}=\vec{\rho} \hat{q}}} F_{\gamma, \beta, G \hat{p}}\left(\vec{\rho}_{G \hat{p}}||_{A^{\hat{p}}}^{\left(\ell_{0}\right)}\right)-\inf _{\substack{\vec{\rho}_{G \hat{p}}: \eta\left(\vec{\rho}_{G \hat{p}}\right)=a^{p} \\
\vec{\rho}_{A \hat{q}}=\vec{\rho} \hat{p}}} F_{\gamma, \beta, G \hat{p}}\left(\vec{\rho}_{G \hat{p}} \mid \vec{\xi}_{A^{p}}^{\left(\ell_{0}\right)}\right)\right]\right\}
\end{aligned}
$$

Proof of Theorem 8.2. For each $\mathcal{D}^{\ell_{+, \gamma}}$-measurable cube $C$ in $\operatorname{sp}(\Gamma)$, at least one of the two following events occurs (recall definition (3.11)):

(a) there is $x$ in $\Gamma$ such that $C_{x}^{\ell_{+, \gamma}} \sim C$ and $\eta_{x}^{\ell_{-, \gamma}}=0$.

(b) there are $x_{1}, x_{2}$ in $\Gamma$ such that $C_{x_{1}}^{\ell_{+}, \gamma} \sim C, C_{x_{2}}^{\ell_{+}, \gamma} \sim C, C_{x_{1}, \gamma}^{\ell_{-, \gamma}} \sim C_{x_{2}}^{\ell_{-}, \gamma}$ and $\eta_{x_{1}, \gamma}^{\ell_{-, \gamma}} \neq$ $0, \eta_{x_{2}, \gamma}^{\ell_{-}} \neq 0, \eta_{x_{1}, \gamma}^{\ell_{-}} \neq \eta_{x_{2}, \gamma}^{\ell_{-, \gamma}}$.

Let $N_{\Gamma}$ the number of cubes of $\mathcal{D}^{\ell_{+}, \gamma}$ contained in $\operatorname{sp}(\Gamma)$. Since a single event can be associated to at most $3^{d}$ cubes, there are at least $3^{-d} N_{\Gamma}$ distinct and nonintersecting events. Let consider a maximal family of nonintersecting events and denote by $\left(x_{0}^{i}\right)_{i \in I_{a}}$, respectively $\left(x_{1}^{j}, x_{2}^{j}\right)_{j \in I_{b}}$ a set of points (respectively a set of pairs of points) characterizing the events of type $a$ (respectively of type $b$ ). We also define

$$
\begin{aligned}
& B_{a}=\bigsqcup_{i \in I_{a}} C_{x_{0}^{i}, \gamma}^{\ell_{-}} \\
& B_{b}=\bigsqcup_{j \in I_{b}}\left(C_{x_{1}^{\ell_{-}, \gamma}}^{\ell_{1}} \sqcup C_{x_{2}^{\ell_{-, \gamma}}}^{\ell^{j}}\right)
\end{aligned}
$$

We have

$$
\left|I_{a}\right|+\left|I_{b}\right| \geq \frac{1}{3^{d}} N_{\Gamma}
$$




$$
\left|B_{a}\right|+\left|B_{b}\right| \geq \frac{1}{3^{d}}\left(\frac{\ell_{-, \gamma}}{\ell_{+, \gamma}}\right)^{d}|\Gamma|
$$

We separate $G^{\hat{p}}$ in two components $G^{\hat{p}}=G_{1} \sqcup G_{2}$,

$$
\begin{aligned}
& G_{1}=\delta_{\text {out }}^{\ell+, \gamma}\left(A^{\hat{p}}\right) \sqcap \operatorname{sp}(\Gamma) \\
& G_{2}=G^{\hat{p}} \backslash G_{1}
\end{aligned}
$$

and write for all $\vec{\rho}$ in $\mathcal{R}(\Gamma)$

$$
F_{\gamma, \beta, G^{\hat{p}}}\left(\vec{\rho} \mid \vec{\xi}_{A^{\hat{p}}}^{\left(\ell_{0}\right)}\right)=F_{\gamma, \beta, G_{1}}\left(\vec{\rho}_{G_{1}} \mid \vec{\xi}_{A^{\hat{p}}}^{\left(\ell_{0}\right)}\right)+F_{\gamma, \beta, G_{2}}\left(\vec{\rho}_{G_{2}} \mid \vec{\rho}_{G_{1}}\right)
$$

We first apply corollary B.5 with $\Lambda=G_{1} \backslash \delta_{\text {in }}^{\gamma^{-1}}\left(G_{1}\right), \rho^{*}=\rho$ and $u=0$. Thus there are positive constants $\omega$ and $c_{\omega}$ and there exists $\psi \in \mathcal{R}(\Gamma)$ such that

$$
F_{\gamma, \beta, G_{1}}\left(\vec{\rho}_{G_{1}} \mid \vec{\xi}_{A^{\hat{p}}}^{\left(\ell_{0}\right)}\right) \geq F_{\gamma, \beta, G_{1}}\left(\vec{\psi}_{G_{1}} \mid \vec{\xi}_{A^{\hat{p}}}^{\left(\ell_{0}\right)}\right)-c_{\omega}\left|G_{1}\right| e^{-\omega \ell_{+, \gamma} / 4}
$$

with

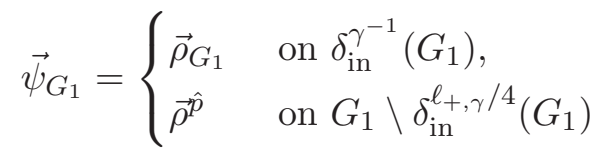

and

$$
F_{\gamma, \beta, G_{2}}\left(\vec{\rho}_{G_{2}} \mid \vec{\rho}_{G_{1}}\right)=F_{\gamma, \beta, G_{2}}\left(\vec{\rho}_{G_{2}} \mid \vec{\psi}_{G_{1}}\right)
$$

Thus we have

$$
F_{\gamma, \beta, G \hat{p}}\left(\vec{\rho} \mid \vec{\xi}_{A^{\hat{p}}}^{\left(\ell_{0}\right)}\right) \geq F_{\gamma, \beta, G \hat{p}}\left(\vec{\psi} \mid \vec{\xi}_{A^{\hat{p}}}^{\left(\ell_{0}\right)}\right)-c_{\omega}\left|G_{1}\right| e^{-\omega \ell_{+, \gamma} / 4}
$$

For $i \in I_{a}$, we write $C_{i}=C_{x_{i}, \gamma}^{\ell-\gamma}$ as a shorthand notation, and define the function $\vec{\psi}^{0}$ on $G^{\hat{p}}$ as

$$
\vec{\psi}^{0}(r)= \begin{cases}\frac{1}{\left|C_{i}\right|} \int_{C_{r}^{\ell-, \gamma}} \vec{\psi}\left(r^{\prime}\right) d r^{\prime} & \text { if } r \in B_{a} \\ \psi(r) & \text { otherwise. }\end{cases}
$$


From the definition of a contour, it follows that $\operatorname{dist}\left(B_{a}, \operatorname{sp}(\Gamma)^{c}\right) \geq \gamma^{-1}$. Thus the energy terms in the free energies of $\vec{\psi}$ and $\vec{\psi}^{0}$ differ by the quantity

$$
\begin{aligned}
& \left|U_{\gamma, G^{\hat{p}}}\left(\vec{\psi} \mid \vec{\xi}_{A^{\hat{p}}}^{\left(\ell_{0}\right)}\right)-U_{\gamma, G^{\hat{p}}}\left(\vec{\psi}^{0} \mid \vec{\xi}_{A^{\hat{p}}}^{\left(\ell_{0}\right)}\right)\right| \\
& =\frac{1}{2}\left|\int_{\operatorname{sp}(\Gamma) \times \operatorname{sp}(\Gamma)} J_{\gamma}(x, y) \vec{\psi}(x) \cdot \vec{\psi}(y) d x d y-\int_{\mathrm{sp}(\Gamma) \times \operatorname{sp}(\Gamma)} J_{\gamma}(x, y) \vec{\psi}^{0}(x) \cdot \vec{\psi}^{0}(y) d x d y\right| \\
& =\frac{1}{2}\left|\int_{\operatorname{sp}(\Gamma) \times \operatorname{sp}(\Gamma)} J_{\gamma}(x, y)\left(\vec{\psi}(x)+\vec{\psi}^{0}(x)\right) \cdot\left(\vec{\psi}(y)-\vec{\psi}^{0}(y)\right) d x d y\right| \\
& =\frac{1}{2} \sum_{i \in I_{a}}\left|\frac{1}{\left|C_{i}\right|} \int_{\operatorname{sp}(\Gamma) \times C_{i}} d x d y J_{\gamma}(x, y)\left(\vec{\psi}(x)+\vec{\psi}^{0}(x)\right) \cdot \int_{C_{i}} d y^{\prime}\left(\vec{\psi}(y)-\vec{\psi}\left(y^{\prime}\right)\right)\right| \\
& =\frac{1}{2} \sum_{i \in I_{a}}\left|\frac{1}{\left|C_{i}\right|} \int_{\operatorname{sp}(\Gamma) \times C_{i}} d x d y\left(\vec{\psi}(x)+\vec{\psi}^{0}(x)\right) \cdot \vec{\psi}(y) \int_{C_{i}} d y^{\prime}\left(J_{\gamma}(x, y)-J_{\gamma}\left(x, y^{\prime}\right)\right)\right| \\
& \leq \frac{1}{2} \sum_{i \in I_{a}} \frac{1}{\left|C_{i}\right|} \int_{\operatorname{sp}(\Gamma) \times C_{i}} d x d y\left|\left(\vec{\psi}(x)+\vec{\psi}^{0}(x)\right) \cdot \vec{\psi}(y)\right| \int_{C_{i}} d y^{\prime}\left|J_{\gamma}(x, y)-J_{\gamma}\left(x, y^{\prime}\right)\right| \\
& \leq 2^{d} \sqrt{d} \ell_{-, \gamma} \gamma\|\nabla \mathcal{J}\|_{\infty}\left|B_{a}\right|
\end{aligned}
$$

where in the last inequality we used

$$
\left|\left(\vec{\psi}(x)+\vec{\psi}^{0}(x)\right) \cdot \vec{\psi}(y)\right| \leq 2
$$

and

$$
\left|J_{\gamma}(x, y)-J_{\gamma}\left(x, y^{\prime}\right)\right| \leq \sqrt{d} \ell_{-, \gamma} \gamma^{d+1}\|\nabla \mathcal{J}\|_{\infty} \mathbf{1}_{\left\{|x-y| \leq 2 \gamma^{-1}\right\}}
$$

that for all $y, y^{\prime}$ in $C_{i}$. By concavity of the entropy, $I(\vec{\psi}) \leq I\left(\vec{\psi}^{0}\right)$ and we get

$$
F_{\gamma, \beta, G \hat{p}}\left(\vec{\psi} \mid \vec{\xi}_{A^{\hat{p}}}^{\left(\ell_{0}\right)}\right) \geq F_{\gamma, \beta, G \hat{p}}\left(\vec{\psi}^{0} \mid \vec{\xi}_{A^{\hat{p}}}^{\left(\ell_{0}\right)}\right)-2^{d} \sqrt{d} \ell_{-, \gamma} \gamma\|\nabla \mathcal{J}\|_{\infty}\left|B_{a}\right|
$$

We look for a lower bound of the free energy of $\vec{\psi}^{0}$. We again divide $G^{\hat{p}}$ in two parts $G^{\hat{p}}=G_{1}^{\prime} \sqcup G_{2}^{\prime}$ where

$$
\begin{aligned}
& G_{1}^{\prime}=\delta_{\text {out }}^{\ell+, \gamma / 2}\left(A^{\hat{p}}\right) \sqcap \operatorname{sp}(\Gamma) \\
& G_{2}^{\prime}=G \backslash G_{1}^{\prime}
\end{aligned}
$$

We have

$$
F_{\gamma, \beta, G^{\hat{p}}}\left(\vec{\psi}^{0} \mid \vec{\xi}_{A^{\hat{p}}}^{\left(\ell_{0}\right)}\right)=F_{\gamma, \beta, G_{1}^{\prime}}\left(\vec{\psi}_{G_{1}^{\prime}}^{0} \mid \vec{\xi}_{A^{\hat{p}}}^{\left(\ell_{0}\right)}\right)+F_{\gamma, \beta, G_{2}^{\prime}}\left(\vec{\psi}_{G_{2}^{\prime}}^{0} \mid \vec{\psi}_{G_{1}^{\prime}}^{0}\right)
$$

We write the second term as:

$$
\begin{aligned}
& F_{\gamma, \beta, G_{2}^{\prime}}\left(\vec{\psi}_{G_{2}^{\prime}}^{0} \mid \vec{\psi}_{G_{1}^{\prime}}^{0}\right)=\int_{G_{2}^{\prime}} d x \phi_{\beta}^{m f}\left(\vec{\psi}^{0}(x)\right)+\frac{1}{4} \int_{G_{2}^{\prime} \times G_{2}^{\prime}} d x d y J_{\gamma}(x, y)\left|\vec{\psi}^{0}(x)-\vec{\psi}^{0}(y)\right|^{2} \\
& -\int_{G_{2}^{\prime} \times G_{1}^{\prime}} d x d y J_{\gamma}(x, y)\left(\vec{\psi}^{0}(x) \cdot \vec{\psi}^{0}(y)\right)+\frac{1}{2} \int_{G_{2}^{\prime} \times G_{2}^{\prime c}} d x d y J_{\gamma}(x, y)\left|\vec{\psi}^{0}(x)\right|^{2}
\end{aligned}
$$


The last two terms can be calculated since, by (8.17), $\vec{\psi}^{0}$ is constant and equal to $\vec{\rho}^{\hat{p}}$ on both $\delta_{\text {out }}^{\gamma^{-1}}\left(G_{1}^{\prime}\right) \sqcap G_{2}^{\prime}$ and $G_{1}^{\prime} \sqcap \delta_{\text {out }}^{\gamma^{-1}}\left(G_{2}^{\prime}\right)$, and equal to $\vec{\rho}^{\hat{q}}$ on $A^{\hat{q}}, \hat{q} \neq \hat{p}$. We get:

$$
\begin{array}{r}
F_{\gamma, \beta, G_{2}^{\prime}}\left(\vec{\psi}_{G_{2}^{\prime}}^{0} \mid \vec{\psi}_{G_{1}^{\prime}}^{0}\right)=\int_{G_{2}^{\prime}} d x \phi_{\beta}^{\mathrm{mf}}\left(\vec{\psi}^{0}(x)\right)+\frac{1}{4} \int_{G_{2}^{\prime} \times G_{2}^{\prime}} d x d y J_{\gamma}(x, y)\left|\vec{\psi}^{0}(x)-\vec{\psi}^{0}(y)\right|^{2} \\
+\frac{1}{2} \sum_{\hat{q} \neq \hat{p}} \int_{G_{2}^{\prime} \times\left(\operatorname{Int}^{\hat{q}}(\Gamma) \backslash A^{\hat{q}}\right)} d x d y J_{\gamma}(x, y)\left|\vec{\rho}^{\hat{q}}\right|^{2}-\frac{1}{2} \int_{G_{2}^{\prime} \times G_{1}^{\prime}} d x d y J_{\gamma}(x, y)\left|\vec{\rho}^{\hat{p}}\right|^{2}
\end{array}
$$

Since $B_{b} \sqsubset G_{2}^{\prime}$, the second term can be bounded by the contributions of the $b$-events. We have the following estimate:

$$
\begin{aligned}
& \frac{1}{4} \int_{G_{2}^{\prime} \times G_{2}^{\prime}} d x d y J_{\gamma}(x, y)\left|\vec{\psi}^{0}(x)-\vec{\psi}^{0}(y)\right|^{2} \\
& \geq \frac{1}{4} \sum_{j \in I_{b}}\left(\int_{G_{2}^{\prime} \times C_{j}} d x d y J_{\gamma}(x, y)\left|\vec{\psi}^{0}(x)-\vec{\psi}^{0}(y)\right|^{2}+\int_{G_{2}^{\prime} \times C_{j}^{\prime}} d x d y^{\prime} J_{\gamma}\left(x, y^{\prime}\right)\left|\vec{\psi}^{0}(x)-\vec{\psi}^{0}\left(y^{\prime}\right)\right|^{2}\right) \\
& =\frac{1}{4} \sum_{j \in I_{b}} \frac{1}{\left|C_{j}\right|} \int_{G_{2}^{\prime}} d x \int_{C_{j} \times C_{j}^{\prime}} d y d y^{\prime}\left(J_{\gamma}(x, y)\left|\vec{\psi}^{0}(x)-\vec{\psi}^{0}(y)\right|^{2}+J_{\gamma}\left(x, y^{\prime}\right)\left|\vec{\psi}^{0}(x)-\vec{\psi}^{0}\left(y^{\prime}\right)\right|^{2}\right)
\end{aligned}
$$

where we used the notations $C_{j}=C_{x_{1}^{j}, \gamma}^{\ell_{-}}, C_{j}^{\prime}=C_{x_{2}^{j}}^{\ell_{-, \gamma}}$ for all $j \in I_{b}$. Using now the fact that for all $\left(y, y^{\prime}\right)$ in $C_{j} \times C_{j}^{\prime}$,

$$
\left|J_{\gamma}(x, y)-J_{\gamma}\left(x, y^{\prime}\right)\right| \leq 2 \sqrt{d} \ell_{-, \gamma} \gamma^{d+1}\|\nabla \mathcal{J}\|_{\infty} \mathbf{1}_{\left\{|x-y| \leq 2 \gamma^{-1}\right\}}
$$

we get

$$
\begin{aligned}
& \frac{1}{4} \int_{G_{2}^{\prime} \times G_{2}^{\prime}} d x d y J_{\gamma}(x, y)\left|\vec{\psi}^{0}(x)-\vec{\psi}^{0}(y)\right|^{2} \\
& \geq \frac{1}{4} \sum_{j \in I_{b}} \int_{G_{2}^{\prime}} d x \frac{1}{2\left|C_{j}\right|} \\
& \quad \int_{C_{j} \times C_{j}^{\prime}} d y d y^{\prime}\left(J_{\gamma}(x, y)+J_{\gamma}\left(x, y^{\prime}\right)\right)\left(\left|\vec{\psi}^{0}(x)-\vec{\psi}^{0}(y)\right|^{2}+\left|\vec{\psi}^{0}(x)-\vec{\psi}^{0}\left(y^{\prime}\right)\right|^{2}\right) \\
& \quad-2^{d-1} \sqrt{d} \ell_{-, \gamma} \gamma\|\nabla \mathcal{J}\|_{\infty}\left|B_{b}\right|
\end{aligned}
$$

Now using successively the inequality

$$
|a-b|^{2}+|a-c|^{2} \geq \frac{1}{2}|b-c|^{2}
$$

and $\operatorname{dist}\left(B_{b}, G_{2}^{\prime c}\right) \geq \gamma^{-1}$, we can sum over the $x$ variable to obtain

$$
\begin{aligned}
& \frac{1}{4} \int_{G_{2}^{\prime} \times G_{2}^{\prime}} d x d y J_{\gamma}(x, y)\left|\vec{\psi}^{0}(x)-\vec{\psi}^{0}(y)\right|^{2} \\
& \geq \frac{1}{8} \sum_{j \in I_{b}} \int_{G_{2}^{\prime}} d x \frac{1}{2\left|C_{j}\right|} \int_{C_{j} \times C_{j}^{\prime}} d y d y^{\prime}\left(J_{\gamma}(x, y)+J_{\gamma}\left(x, y^{\prime}\right)\right)\left|\vec{\psi}^{0}(y)-\vec{\psi}^{0}\left(y^{\prime}\right)\right|^{2} \\
& -2^{d-1} \sqrt{d} \ell_{-, \gamma} \gamma\|\nabla \mathcal{J}\|_{\infty}\left|B_{b}\right| \\
& \geq \frac{1}{8} \sum_{j \in I_{b}} \frac{1}{\left|C_{j}\right|} \int_{C_{j} \times C_{j}^{\prime}} d y d y^{\prime}\left|\vec{\psi}^{0}(y)-\vec{\psi}^{0}\left(y^{\prime}\right)\right|^{2}-2^{d-1} \sqrt{d} \ell_{-, \gamma} \gamma\|\nabla \mathcal{J}\|_{\infty}\left|B_{b}\right|
\end{aligned}
$$


We finally get

$$
\begin{aligned}
& \frac{1}{4} \int_{G_{2}^{\prime} \times G_{2}^{\prime}} d x d y J_{\gamma}(x, y)\left|\vec{\psi}^{0}(x)-\vec{\psi}^{0}(y)\right|^{2} \\
& \geq \frac{1}{8} \sum_{j \in I_{b}} \frac{1}{\left|C_{j}\right|}\left|\int_{C_{j}} d y \vec{\psi}^{0}(y)-\int_{C_{j}^{\prime}} d y^{\prime} \vec{\psi}^{0}\left(y^{\prime}\right)\right|^{2}-2^{d-1} \sqrt{d} \ell_{-, \gamma} \gamma\|\nabla \mathcal{J}\|_{\infty}\left|B_{b}\right|
\end{aligned}
$$

Now collecting all estimates (8.19), (8.23) and (8.33), we get for all $\vec{\rho}$ in $\mathcal{R}(\Gamma)$ :

$$
\begin{aligned}
& F_{\gamma, \beta, G^{\hat{p}}}\left(\vec{\rho} \mid \vec{\xi}_{A^{\hat{p}}}^{\left(\ell_{0}\right)}\right) \geq F_{\gamma, \beta, G_{1}^{\prime}}\left(\vec{\psi}_{G_{1}^{\prime}}^{0} \mid \vec{\xi}_{A^{\hat{p}}}^{\left(\ell_{0}\right)}\right)+\sum_{\hat{q} \neq \hat{p}} \mathcal{I}_{A^{\hat{q}}}\left|\vec{\rho}^{q}\right|^{2}-\frac{1}{2} \int_{G_{2}^{\prime} \times G_{1}^{\prime}} d x d y J_{\gamma}(x, y)\left|\vec{\rho}^{\hat{p}}\right|^{2} \\
& +\int_{G_{2}^{\prime}} d x \phi_{\beta}^{\mathrm{mf}}\left(\vec{\psi}^{0}(x)\right)+\frac{1}{8} \sum_{j \in I_{b}} \frac{1}{\left|C_{j}\right|}\left|\int_{C_{j}} d y^{\prime} \vec{\psi}^{0}\left(y^{\prime}\right)-\int_{C_{j}^{\prime}} d y^{\prime \prime} \vec{\psi}^{0}\left(y^{\prime \prime}\right)\right|^{2} \\
& -c_{\omega}\left|G_{1}\right| e^{-\omega \ell_{+, \gamma} / 4}-2^{d} \sqrt{d} \ell_{-, \gamma} \gamma\|\nabla \mathcal{J}\|_{\infty}\left|B_{a}\right|-2^{d-1} \sqrt{d} \ell_{-, \gamma} \gamma\|\nabla \mathcal{J}\|_{\infty}\left|B_{b}\right|
\end{aligned}
$$

Using the definitions of $B_{a}$ and $B_{b}$ and the results of appendix A, we have for all $\beta$ such that $\beta-\beta_{c}^{\mathrm{mf}} \leq c_{b} \gamma^{\frac{1}{2}}$,

$$
\phi_{\beta}^{m f}\left(\vec{\psi}^{0}(x)\right) \geq \phi_{\beta}^{m f}\left(\vec{\rho}^{\hat{p}}\right)+\left(\frac{Q}{\beta}-1\right) \gamma^{2 a}-c \gamma^{\frac{1}{2}}
$$

for all $x$ in $B_{a}$ and

$$
\frac{1}{\left|C_{j}\right|^{2}}\left|\int_{C_{j}} d y \vec{\psi}^{0}(y)-\int_{C_{j}^{\prime}} d y^{\prime} \vec{\psi}^{0}\left(y^{\prime}\right)\right|^{2} \geq\left(\frac{Q-1}{Q}-2 \gamma^{a}\right)^{2}
$$

on all $j$ in $I_{b}$ Hence

$$
\begin{aligned}
& F_{\gamma, \beta, G \hat{p}}\left(\vec{\rho} \mid \vec{\xi}_{A^{\hat{p}}}^{\left(\ell_{0}\right)}\right) \geq F_{\gamma, \beta, G_{1}^{\prime}}\left(\vec{\psi} \mid \vec{\xi}_{A^{\hat{p}}}^{\left(\ell_{0}\right)}\right)+\left|G_{2}^{\prime}\right| \phi_{\beta}^{\mathrm{mf}}\left(\vec{\rho}^{\hat{p}}\right)+\sum_{\hat{q} \neq \hat{p}} \mathcal{I}_{A^{\hat{q}}}\left|\vec{\rho}^{q}\right|^{2}-\frac{1}{2} \int_{G_{2}^{\prime} \times G_{1}^{\prime}} d x d y J_{\gamma}(x, y)\left|\vec{\rho}^{\hat{p}}\right|^{2} \\
& +\left(\frac{Q}{\beta}-1\right) \gamma^{2 a}\left|B_{a}\right|+\frac{1}{4}\left(\frac{Q-1}{2 Q}-\gamma^{a}\right)^{2}\left|B_{b}\right| \\
& -c_{\omega}\left|G_{1}\right| e^{-\omega \ell_{+, \gamma} / 4}-2^{d} \sqrt{d} \ell_{-, \gamma} \gamma\|\nabla \mathcal{J}\|_{\infty}\left|B_{a}\right|-c \gamma^{\frac{1}{2}}-2^{d-1} \sqrt{d} \ell_{-, \gamma} \gamma\|\nabla \mathcal{J}\|_{\infty}\left|B_{b}\right|
\end{aligned}
$$

Now for $a<\min \left(\frac{1}{4}, \frac{\alpha}{2}\right)$, we may choose $\gamma$ so that the last three error terms are a fraction of the respective gain terms. In addition, since we have both $\left|B_{a}\right|+\left|B_{b}\right| \geq \frac{1}{3^{d}}\left(\frac{\ell_{-, \gamma}}{\ell_{+, \gamma}}\right)^{d}|\Gamma|$ and $G_{1} \sqsubset \Gamma$, the remaining error term can be also compensated for $\gamma$ small enough. We define $\bar{\gamma}$ as the largest value of $\gamma$ such that the following inequalities hold simultaneously

$$
\begin{aligned}
& 2^{d-1} \sqrt{d} \ell_{-, \gamma} \gamma\|\nabla \mathcal{J}\|_{\infty} \leq \frac{1}{3}\left(\frac{Q-1}{2 Q}-\gamma^{a}\right)^{2} \\
& 2^{d} \sqrt{d} \ell_{-, \gamma} \gamma\|\nabla \mathcal{J}\|_{\infty} \leq \frac{1}{3}\left(\frac{Q}{\beta}-1\right) \gamma^{2 a} \\
& c_{\omega} e^{-\omega \ell_{+, \gamma} / 4} \leq \frac{1}{3}\left(\frac{Q}{\beta}-1\right) \gamma^{2 a} \\
& \frac{1}{4}\left(\frac{Q-1}{2 Q}-\gamma^{a}\right)^{2} \geq \frac{1}{3}\left(\frac{Q}{\beta}-1\right) \gamma^{2 a}
\end{aligned}
$$


For all $\gamma \leq \bar{\gamma}$, we have

$$
\begin{aligned}
& F_{\gamma, \beta, G \hat{p}}\left(\vec{\rho} \mid \vec{\xi}_{A^{\hat{p}}}^{\left(\ell_{0}\right)}\right) \geq F_{\gamma, \beta, G_{1}^{\prime}}\left(\vec{\psi} \mid \vec{\xi}_{A^{\hat{p}}}^{\left(\ell_{0}\right)}\right)+\left|G_{2}^{\prime}\right| \phi_{\beta}^{m f}\left(\vec{\rho}^{\hat{p}}\right)-\frac{1}{2} \int_{G_{2}^{\prime} \times G_{1}^{\prime}} d x d y J_{\gamma}(x, y)\left|\vec{\rho}^{\hat{p}}\right|^{2} \\
& +\sum_{\hat{q} \neq \hat{p}} \mathcal{I}_{A^{\hat{q}}\left|\vec{\rho}^{q}\right|^{2}}+\frac{1}{3^{d+1}}\left(\frac{Q}{\beta}-1\right) \gamma^{2 a}\left(\frac{\ell_{-, \gamma}}{\ell_{+, \gamma}}\right)^{d}|\Gamma|
\end{aligned}
$$

Now consider the function $\vec{\varphi}$ in $L^{\infty}\left(G^{\hat{p}}, S_{Q}\right)$ defined as

$$
\vec{\varphi}(r)= \begin{cases}\vec{\psi}(r) & r \in G_{1}^{\prime}, \\ \vec{\rho}^{\hat{p}} & r \in G_{2}^{\prime} .\end{cases}
$$

$\vec{\varphi}(r)$ belongs clearly to $\mathcal{R}(\not \Gamma)$, and since $\vec{\varphi}(r)=\vec{\rho}^{\hat{p}}$ on $G_{1}^{\prime} \sqcap \delta_{\text {out }}^{\gamma^{-1}}\left(G_{2}^{\prime}\right)$, its free energy reads

$$
\begin{aligned}
& F_{\gamma, \beta, G^{\hat{p}}}\left(\vec{\varphi} \mid \vec{\xi}_{A^{\hat{p}}}^{\left(\ell_{0}\right)}\right)=F_{\gamma, \beta, G_{1}^{\prime}}\left(\vec{\varphi} \mid \vec{\xi}_{A^{\hat{p}}}^{\left(\ell_{0}\right)}\right) \\
& +\left|G_{2}^{\prime}\right| \phi_{\beta}^{m f}\left(\vec{\rho}^{\hat{p}}\right)-\frac{1}{2} \int_{G_{2}^{\prime} \times G_{1}^{\prime}} d x d y J_{\gamma}(x, y)\left|\vec{\rho}^{\hat{p}}\right|^{2}+\sum_{\hat{q} \neq \hat{p}} \mathcal{I}_{A^{\hat{q}}}\left|\vec{\rho}^{p}\right|^{2}
\end{aligned}
$$

Hence we have for all $\vec{\rho}$ in $\mathcal{R}(\Gamma)$,

$$
\begin{aligned}
& F_{\gamma, \beta, G \hat{p}}\left(\vec{\rho} \mid \vec{\xi}_{A^{\hat{p}}}^{\left(\ell_{0}\right)}\right) \geq F_{\gamma, \beta, G^{\hat{p}}}\left(\vec{\varphi} \mid \vec{\xi}_{A^{\hat{p}}}^{\left(\ell_{0}\right)}\right) \\
& +\sum_{\hat{q} \neq \hat{p}} \mathcal{I}_{A^{\hat{q}}}\left(\left|\hat{\rho}^{\hat{q}}\right|^{2}-\left|\vec{\rho}^{\hat{p}}\right|^{2}\right)+\frac{1}{3^{d+1}}\left(\frac{Q}{\beta}-1\right) \gamma^{2 a}\left(\frac{\ell_{-, \gamma}}{\ell_{+, \gamma}}\right)^{d}|\Gamma|
\end{aligned}
$$

and since $\vec{\varphi}$ is in $\mathcal{R}(\not T)$,

$$
F_{\gamma, \beta, G \hat{p}}\left(\vec{\varphi} \mid \vec{\xi}_{A^{\hat{p}}}^{\left(\ell_{0}\right)}\right) \geq \inf _{\rho \in \mathcal{R}(\not \bar{~})} F_{\gamma, \beta, G \hat{p}}\left(\vec{\rho} \mid \vec{\xi}_{A^{\hat{p}}}^{\left(\ell_{0}\right)}\right)
$$

Taking now the infimum over $\mathcal{R}(\Gamma)$ in (8.37), we get for all $\gamma \leq \bar{\gamma}$,

$$
\begin{aligned}
& \inf _{\vec{\rho} \in \mathcal{R}(\Gamma)} F_{\gamma, \beta, G \hat{p}}\left(\vec{\rho} \mid \vec{\xi}_{A^{\hat{p}}}^{\left(\ell_{0}\right)}\right) \geq \inf _{\rho \in \mathcal{R}(\not \overline{)})} F_{\gamma, \beta, G \hat{p}}\left(\vec{\rho} \mid \vec{\xi}_{A^{\hat{p}}}^{\left(\ell_{0}\right)}\right) \\
& +\sum_{\hat{q} \neq \hat{p}} \mathcal{I}_{A^{\hat{q}}}\left(\left|\hat{\rho}^{\hat{q}}\right|^{2}-\left|\vec{\rho}^{\hat{p}}\right|^{2}\right)+c_{f} \gamma^{2 a}\left(\frac{\ell_{-, \gamma}}{\ell_{+, \gamma}}\right)^{d}|\Gamma|
\end{aligned}
$$

with

$$
c_{f}=\frac{1}{3^{d+1}}\left(\frac{Q}{\beta}-1\right)
$$

\section{Acknowledgments}

We thank Marzio Cassandro, Roberto Fernandez, Joel Lebowitz and Errico Presutti for many helpful discussions. T.G. thanks the warm hospitality of the Mathematics departments of Universities of Rome 2 and l'Aquila where most of the work has been done. 


\section{Appendix A. Mean Field Model}

In this appendix, we review briefly the mean field theory of the Potts model [30], and derive the various quantities needed in the rest of the paper.

We consider a $Q$-state Potts model defined on a complete graph with $N$ sites and derive its behavior in the large $N$ limit. A variable $\sigma_{i}, \sigma_{i} \in[1, \cdots, Q]$, is attached to each site $i$ of the graph so that the space of configurations is $\Omega_{N}=[1, \cdots, Q]^{N}$. The mean field Hamiltonian on $\Omega_{N}$ is:

$$
H^{\mathrm{mf}}(\sigma):=-\frac{1}{2 N} \sum_{i \neq j} \mathbf{1}_{\left\{\sigma_{i}=\sigma_{j}\right\}}
$$

$H^{\mathrm{mf}}$ is invariant under any permutation of sites so that its value on a given configuration depends only on the number of sites with color $q$, says $N_{q}, q \in[1, \cdots, Q]$. The partition function of the model is thus:

$$
Z_{N, \beta}=\sum_{\left\{N_{q}\right\}: \sum N_{q}=N} \frac{N !}{\prod_{q} N_{q} !} e^{\frac{\beta}{2 N} \sum_{q} N_{q}\left(N_{q}-1\right)}
$$

For $N$ large, $Z_{N, \beta}$ is dominated by the configurations which realize the minimum of the free energy density $\phi_{\beta}^{\mathrm{mf}}$ defined on $S_{Q}=\left\{\vec{\rho} \in \mathbb{R}_{+}^{Q}, \sum_{q} \rho_{q}=1\right\}$ as

$$
\phi_{\beta}^{\mathrm{mf}}(\vec{\rho})=-\frac{1}{2} \sum_{q} \rho_{q}^{2}+\frac{1}{\beta} \sum_{q} \rho_{q} \ln \left(\rho_{q}\right)
$$

with the correspondence $\rho_{q}=\frac{N_{q}}{N}$. In fact, for our present purpose, we are also interested in all local minimizers of $\phi_{\beta}^{\mathrm{mf}}$, which appear to be of two kinds: one "disordered" (or uniform) state in which all colors have the same density and $Q$ degenerated "ordered" (or colored) states in which one color dominates. As can be expected, the first one exists for small values of $\beta$, while the other $Q$ exist only for $\beta$ large enough. In addition, there is a critical value of $\beta$ which determines which kind of local minimizer is the actual absolute minimizer for $\phi_{\beta}^{\mathrm{mf}}$. We make these statements precise in the two following theorems. We first characterize all local minimizers:

Theorem A.1. For all $Q>2$, there exists $\beta_{0}<Q$ such that the mean field free energy $\phi_{\beta}^{\mathrm{mf}}$ A.2. on $S_{Q}$ has exactly:

- 1 local minimizer for $\beta \leq \beta_{0}$,

- $Q+1$ local minimizers for $\beta_{0}<\beta<Q$, and

- $Q$ local minimizers for $\beta \geq Q$.

These minimizers are of two kinds and characterized as follows:

- For all $\beta<Q$, the uniform state $\vec{\rho}^{(-1)}$, with components

$$
\rho_{q}^{(-1)}=\frac{1}{Q} \quad \text { for all } q \in\{1, \cdots, Q\}
$$


and free energy

$$
\phi_{\beta}^{\mathrm{mf}}\left(\vec{\rho}^{(-1)}\right)=\frac{-1}{2 Q}-\frac{1}{\beta} \log (Q)
$$

- For all $\beta>\beta_{0}, Q$ colored states $\vec{\rho}^{(p)} \equiv \vec{\rho}_{\beta}^{(p)}, p \in\{1, \cdots, Q\}$, with components

$$
\rho_{q}^{(p)}= \begin{cases}\rho_{A} & \text { if } q=p, \\ \rho_{B} & \text { otherwise } .\end{cases}
$$

where $\left(\rho_{A}, \rho_{B}\right), \rho_{A}>\rho_{B}$, is the solution of the set of equations

$$
\begin{aligned}
\frac{\log \left(\rho_{A}\right)-\log \left(\rho_{B}\right)}{\rho_{A}-\rho_{B}} & =\beta \\
\rho_{A}+(Q-1) \rho_{B} & =1
\end{aligned}
$$

which verify

$$
Q \beta \rho_{A} \rho_{B}<1
$$

These $Q$ states are degenerate and have free energy

$$
\phi_{\beta}^{\mathrm{mf}}\left(\vec{\rho}^{(p)}\right)=\frac{-1}{2} Q \rho_{A} \rho_{B}+\frac{1}{2 \beta} \log \left(\rho_{A} \rho_{B}\right)
$$

The mean field first order transition is described in the following

Theorem A.2. For all $Q>2$, there exists a critical value of $\beta$, in $\left(\beta_{0}, Q\right)$,

$$
\beta_{c}^{\mathrm{mf}} \equiv \frac{2(Q-1)}{Q-2} \log (Q-1)
$$

such that $\phi_{\beta}^{\mathrm{mf}}$ has:

- 1 minimizer $\vec{\rho}^{(-1)}$ for all $\beta<\beta_{c}^{\mathrm{mf}}$;

- $Q+1$ minimizers $\vec{\rho}^{(\hat{p})}, \hat{p} \in\{-1,1, \cdots, Q\}$ for $\beta=\beta_{c}^{\mathrm{mf}}$;

- $Q$ minimizers $\vec{\rho}^{(p)}, p \in\{1, \cdots, Q\}$ for $\beta>\beta_{c}^{\mathrm{mf}}$.

Finally, the relevance of local minimizers in our problem arises from their local stability, which is stated in the following theorem:

Theorem A.3. For all $Q>2$ and all $\beta$ in $\left(\beta_{0}, Q\right)$, the map

$$
\vec{\rho} \rightarrow \vec{g}(\vec{\rho})
$$

where

$$
g_{q}(\vec{\rho})=\frac{\exp \left(\beta \rho_{q}\right)}{\sum_{p=1}^{Q} \exp \left(\beta \rho_{p}\right)}
$$

is a contraction around the $Q+1$ local minimizers $\vec{\rho}^{(\hat{p})}$ of the mean field free energy $\phi_{\beta}^{\mathrm{mf}}$. In particular,

$$
\sup _{q, q^{\prime}}\left|\frac{\partial g_{q}(\vec{\rho})}{\partial \rho_{q^{\prime}}}\right| \leq 1-\frac{1}{2 Q}
$$

for all $\vec{\rho}$ such that $\sup _{q}\left|\rho_{q}-\rho_{q}^{\hat{p}}\right| \leq \frac{1}{4 \beta^{2} Q^{2}}$ for some $\hat{p}$. 


\section{PROOF. of Theorem A.1}

We consider the variational problem for $\phi_{\beta}^{\mathrm{mf}}(\vec{\rho})$.

$$
\inf _{\vec{\rho}: \sum_{q=1}^{Q} \rho_{q}=1} \phi_{\beta}^{\operatorname{mf}}(\vec{\rho})=\min _{\vec{\rho}, \lambda} \operatorname{loc}\left[\phi_{\beta}^{\operatorname{mf}}(\vec{\rho})+\lambda\left(\sum_{j=q}^{Q} \rho_{q}-1\right)\right]
$$

where $\lambda$ is a Lagrange parameter associated to the constraint $\sum_{q} \rho_{q}=1$. Since the gradient of the free energy points inward the simplex $S_{Q}$, the (local) minima cannot stay on the boundary of $S_{Q}$ and are thus solutions of the set of equations:

$$
\frac{\partial \phi_{\beta}^{\mathrm{mf}}(\vec{\rho})}{\partial \rho_{q}}+\lambda=0, \quad q=1, \cdots, Q
$$

together with the condition

$$
\sum_{q 1, q 2=1}^{Q} \frac{\partial^{2} \phi_{\beta}^{\mathrm{mf}}(\vec{\rho})}{\partial \rho_{q_{1}} \rho_{q_{2}}} x_{q_{1}} x_{q_{2}} \geq 0
$$

for all $\vec{x} \in \mathbb{R}^{Q}$ such that $\sum_{q=1}^{Q} x_{q}=0$.

Explicitly the first derivatives of the free energy read

$$
\frac{\partial \phi_{\beta}^{\mathrm{mf}}(\vec{\rho})}{\partial \rho_{q}} \equiv 1-\rho_{q}+\frac{1}{\beta}\left(\ln \rho_{q}+1\right)
$$

while the Hessian matrix of $\phi_{\beta}^{\mathrm{mf}}(\vec{\rho})$ is diagonal and

$$
\frac{\partial^{2} \phi_{\beta}^{\mathrm{mf}}(\vec{\rho})}{\partial \rho_{q_{1}} \rho_{q_{2}}}=\delta_{q_{1}, q_{2}}\left(-1+\frac{1}{\beta \rho_{q_{1}}}\right)
$$

As a function of $\rho_{q}$ alone, $\frac{\partial \phi_{\beta}^{\mathrm{mf}}(\vec{\rho})}{\partial \rho_{q}}$ is a strictly concave $C^{\infty}$ function and hence cannot take the same value more than twice. Thus there are two kind of solutions for (A.14), depending on whether $\rho_{q}$ takes one or two values.

The first case correspond to a "disordered" solution $\vec{\rho}^{(-1)}$ in which each color has the same density:

$$
\begin{aligned}
\rho_{q}^{(-1)} & =\frac{1}{Q} \quad \text { for all } q=1, \cdots, Q \\
\phi_{\beta}^{\mathrm{mf}}\left(\vec{\rho}^{(-1)}\right) & =\frac{-1}{2 Q}-\frac{1}{\beta} \ln (Q)
\end{aligned}
$$

Using (A.17), $\vec{\rho}^{(-1)}$ is a local minimum of $\phi_{\beta}^{\mathrm{mf}}(\vec{\rho})$ if and only if $\beta<Q$

In the second case, let $\vec{\rho}$ a vector in $S_{Q}$ which components takes two values, says $\rho_{A}$ and $\rho_{B}$ with $\rho_{A}>\rho_{B}$, and let $n, 0<n<Q$, the number of components equal to $\rho_{A}$ (the remaining $Q-n$ are thus equal to $\left.\rho_{B}\right)$. According to $(\mathbf{A . 1 4}), \vec{\rho}$ is a critical point for $\phi_{\beta}^{\operatorname{mf}}(\vec{\rho})$ in $S_{Q}$ whenever the constraint is satisfied and both $\rho_{A}$ and $\rho_{B}$ are associated to the same 
value of the Lagrange parameter:

$$
\begin{aligned}
& n \rho_{A}+(Q-n) \rho_{B}=1 \\
& \lambda=\rho_{A}-1-\frac{1}{\beta}\left(\ln \rho_{A}+1\right)=\rho_{B}-1-\frac{1}{\beta}\left(\ln \rho_{B}+1\right)
\end{aligned}
$$

However, all such points are not local minima: equation (A.21) implies the relation (A.6) between $\beta, \rho_{A}$ and $\rho_{B}$,

$$
\beta=\frac{\ln \rho_{A}-\ln \rho_{B}}{\rho_{A}-\rho_{B}}
$$

and by concavity of the logarithmic function, we have necessarily

$$
\frac{1}{\rho_{A}}<\beta<\frac{1}{\rho_{B}}
$$

Thus the second derivative of $\phi_{\beta}^{\mathrm{mf}}(\vec{\rho})$ is negative in each direction in which $\rho_{q}=\rho_{A}$. This is obviously not compatible with the condition (A.15) for $n \geq 2$. In the case $n=1$, the constraint A.21 reduces to A.7):

$$
\rho_{A}+(Q-1) \rho_{B}=1
$$

while the condition A.15) can be made explicit using (A.17):

$$
\left(-1+\frac{1}{\beta \rho_{A}}\right)+\frac{1}{Q-1}\left(-1+\frac{1}{\beta \rho_{B}}\right) \geq 0
$$

or equivalently using the constraint (A.24), one gets (A.8):

$$
\beta Q \rho_{A} \rho_{B} \leq 1
$$

We need to find out all triples $\left(\beta, \rho_{A}, \rho_{B}\right)$ which solve simultaneously (A.22), (A.24) and (A.25). For $\rho_{A}$ in $\left(\frac{1}{Q}, 1\right)$, we consider the function $\tilde{\beta}\left(\rho_{A}\right)$ as

$$
\tilde{\beta}\left(\rho_{A}\right)=\frac{\ln \rho_{A}-\ln \rho_{B}}{\rho_{A}-\rho_{B}}
$$

where $\rho_{B}$ is taken implicitly as a function of $\rho_{A}$, through (A.24) Its first derivative reads

$$
\frac{\partial \tilde{\beta}\left(\rho_{A}\right)}{\partial \rho_{A}}=\frac{1}{(Q-1)\left(\rho_{A}-\rho_{B}\right)}\left[\frac{1}{\rho_{A} \rho_{B}}-\beta Q\right]
$$

while the second derivative can be cast in the form

$$
\frac{\partial^{2} \tilde{\beta}\left(\rho_{A}\right)}{\partial \rho_{A}^{2}}=\frac{\varphi\left(\rho_{A}\right)-\varphi\left(\rho_{B}\right)}{(Q-1)^{2}\left(\rho_{A}-\rho_{B}\right)^{3}}
$$

where

$$
\varphi(\rho)=2 Q^{2} \log (\rho)+\frac{4 Q}{\rho}-\frac{1}{\rho^{2}}
$$

One can check that the function $\varphi(\rho)$ is strictly increasing and thus the second derivative of $\tilde{\beta}\left(\rho_{A}\right)$ is always positive. It follows that there exists a unique value $\rho_{0}$ in $\left(\frac{1}{Q}, 1\right)$ so that the first derivative is zero in $\rho_{0}$, strictly negative for $\rho<\rho_{0}$ and strictly positive for $\rho>\rho_{0}$. We define $\beta_{0} \equiv \tilde{\beta}\left(\rho_{0}\right)$. Since equation A.22 is equivalent to $\beta=\tilde{\beta}\left(\rho_{A}\right)$, solutions to A.22 will exist only for $\beta$ in the image of $\tilde{\beta}(\cdot)$, and thus for $\beta \geq \beta_{0}$. Now the condition for a local minimum is equivalent to $\frac{\partial \tilde{\beta}\left(\rho_{A}\right)}{\partial \rho_{A}} \geq 0$ and thus to $\rho_{A} \geq \rho_{0}$. Furthermore the 
function $\tilde{\beta}(\cdot)$ is invertible from $\left(\rho_{0}, 1\right)$ onto $\left(\beta_{0},+\infty\right)$ and therefore, for all $\beta>\beta_{0}$ there is a unique couple $\left(\rho_{A}, \rho_{B}\right)$ for which the vectors A.5 are $Q$ local minima, and there is no "colored" solutions for $\beta<\beta_{0}$. Whenever they exist, those minima are degenerate and their mean field free energy is given by (A.9). We postpone the proof that $\beta_{0}<Q$ at the end of the proof of the next theorem.

Proof. of Theorem A.2 For $\beta$ in $\left(\beta_{0}, Q\right)$, we consider the difference of free energy between ordered and disordered local minima

$$
\Delta\left(\phi_{\beta}^{\mathrm{mf}}\right) \equiv \phi_{\beta}^{\mathrm{mf}}\left(\vec{\rho}^{(p)}\right)-\phi_{\beta}^{\mathrm{mf}}\left(\vec{\rho}^{(-1)}\right)=\frac{1-Q^{2} \rho_{A} \rho_{B}}{2 Q}+\frac{1}{2 \beta} \log \left(Q^{2} \rho_{A} \rho_{B}\right)
$$

We first note that $\beta \Delta\left(\phi_{\beta}^{\mathrm{mf}}\right)$ is a strictly decreasing function of $\beta$ : using (A.26), we have for $\beta$ in $\left(\beta_{0}, Q\right)$,

$$
\frac{\partial}{\partial \beta}\left(\beta \Delta\left(\phi_{\beta}^{\mathrm{mf}}\right)\right)=\left(\frac{\partial \tilde{\beta}}{\partial \rho_{A}}\right)^{-1} \frac{\partial \tilde{\beta} \Delta\left(\phi_{\tilde{\beta}}^{\mathrm{mf}}\right)}{\partial \rho_{A}}=-\frac{\left(1-Q \rho_{A}\right)^{2}}{2 Q(Q-1)}<0
$$

Furthermore, $\Delta\left(\phi_{\beta}^{\mathrm{mf}}\right)$ has one zero for $Q^{2} \rho_{A} \rho_{B}=1$ or equivalently

$$
\rho_{A}=\frac{Q-1}{Q}
$$

This zero is thus necessarily unique and defines a critical value of $\beta$

$$
\beta_{c}^{\mathrm{mf}}=\tilde{\beta}\left(\frac{Q-1}{Q}\right)=\frac{2(Q-1)}{Q-2} \log (Q-1)
$$

We complete the proof by showing that A.25 holds at $\beta=\beta_{c}^{\mathrm{mf}}$. We have for all $Q>2$ :

$$
Q \beta_{c}^{\operatorname{mf}} \rho_{A} \rho_{B}=\frac{2(Q-1)}{Q(Q-2)} \log (Q-1)=\frac{\log (Q-1)}{\sinh (\log (Q-1))}<1
$$

Thus $\beta_{c}^{\mathrm{mf}}>\beta_{0}$. On the other hand, since $\rho_{A} \rho_{B}=Q^{-2}$ at $\beta=\beta_{c}^{\mathrm{mf}}$, A.34 proves also that $\beta_{c}^{\mathrm{mf}}<Q$, and thus $\beta_{0}<\beta_{c}^{\mathrm{mf}}<Q$. This also complete the proof of theorem A.1

\section{PROOF. of Theorem A.3}

Let $\beta$ be in the interval $\left(\beta_{0}, Q\right)$ and consider the map $\vec{\rho} \rightarrow \vec{g}(\vec{\rho})$ defined in (A.12). We have

$$
\frac{\partial g_{q}(\vec{\rho})}{\partial \rho_{q^{\prime}}}=\beta g_{q}(\vec{\rho})\left(\delta_{q, q^{\prime}}-g_{q^{\prime}}(\vec{\rho})\right)
$$

and since $0 \leq g_{q}(\vec{\rho}) \leq 1$, we have a first bound uniform in $\vec{\rho}$ :

$$
\sup _{q, q^{\prime}}\left|\frac{\partial g_{q}}{\partial \rho_{q^{\prime}}}(\vec{\rho})\right| \leq \beta
$$

On the other hand, from A.35, one can also write

$$
\sup _{q, q^{\prime}}\left|\frac{\partial g_{q}}{\partial \rho_{q^{\prime}}}(\vec{\rho})\right| \leq \sup _{q, q^{\prime}}\left|\frac{\partial g_{q}}{\partial \rho_{q^{\prime}}}\left(\vec{\rho}^{\hat{p}}\right)\right|+\sup _{q, q^{\prime}}\left|\frac{\partial g_{q}}{\partial \rho_{q^{\prime}}}(\vec{\rho})-\frac{\partial g_{q}}{\partial \rho_{q^{\prime}}}\left(\vec{\rho}^{\hat{p}}\right)\right|
$$


The first term can be bounded by

$$
\sup _{q, q^{\prime}}\left|\frac{\partial g_{q}}{\partial \rho_{q^{\prime}}}\left(\vec{\rho}^{\hat{p}}\right)\right|=\beta \sup _{q, q^{\prime}}\left|\rho_{q}^{\hat{p}}\left(\delta_{q, q^{\prime}}-\rho_{q^{\prime}}^{\hat{p}}\right)\right| \leq 1-\frac{1}{Q}
$$

where the inequality follows from (A.3) and $\beta \leq Q$ for $\hat{p}=-1$, and from (A.8) and (A.7) for $\hat{p}>0$. The second term in (A.37) can be bounded using (A.36) as

$$
\sup _{q, q^{\prime}}\left|\frac{\partial g_{q}}{\partial \rho_{q^{\prime}}}(\vec{\rho})-\frac{\partial g_{q}}{\partial \rho_{q^{\prime}}}\left(\vec{\rho}^{\hat{p}}\right)\right| \leq 2 \beta \sup _{q}\left|g_{q}(\vec{\rho})-g_{q}\left(\vec{\rho}^{\hat{p}}\right)\right| \leq 2 Q \beta^{2} \sup _{q}\left|\rho_{q}-\rho_{q}^{\hat{p}}\right|
$$

Thus for all $\rho$ such that $\sup _{q}\left|\rho_{q}-\rho_{q}^{\hat{p}}\right| \leq \frac{1}{4 \beta^{2} Q^{2}}$ for some $\hat{p}$ one gets

$$
\sup _{q, q^{\prime}}\left|\frac{\partial g_{q}}{\partial \rho_{q^{\prime}}}(\vec{\rho})\right| \leq 1-\frac{1}{2 Q}
$$

We conclude this appendix by a proof of (6.14):

From equation (A.31) and the definition of $\beta_{c}^{\mathrm{mf}}$ (A.10), one gets explicitly:

$$
\begin{aligned}
\left.\frac{d}{d \beta}\left[P_{\mathrm{mf}, \beta}^{-}-P_{\mathrm{mf}, \beta}^{+}\right]\right|_{\beta=\beta_{c}^{\mathrm{mf}}} & =\left.\frac{1}{\beta_{c}^{\mathrm{mf}}} \frac{\partial}{\partial \beta}\left(\beta \Delta\left(\phi_{\beta}^{\mathrm{mf}}\right)\right)\right|_{\beta=\beta_{c}^{\mathrm{mf}}} \\
& =-\frac{(Q-2)^{2}}{2 Q(Q-1) \beta_{c}^{\mathrm{mf}}}<0
\end{aligned}
$$

\section{Appendix B. Local equilibrium}

The main result of this appendix is the proof that, for suitable values of the temperature, if a density profile is in a neighborhood of an equilibrium value in a region $\Lambda \sqcup \delta_{\text {out }}^{\gamma^{-1}}[\Lambda]$, then it can be made closer to equilibrium inside $\Lambda$ at an exponential rate from its boundary, decreasing the free energy. This result is essentially due to the stability properties of the free energy functionals originating from the contraction property of the map (A.11) around its fixed point. The precise result is stated in the Theorem B.1 below. The proof follows the lines developed in [26, (see also [2]) for Ising model and continuum particle models, and we will stress here only the points specific for our model while we will only sketch the points that are quite analogous to the other cases.

Without lost of generality we study the local equilibrium around the phase " $\hat{p}$ ",

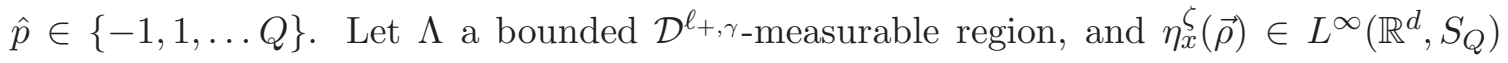
defined analogously as $\eta_{x}(\vec{\rho})$ in (3.9), but with an accuracy parameter, denoted by $\zeta$, that here we leave free

$$
\eta_{x}^{\zeta}(\vec{\rho})= \begin{cases}a_{\hat{p}} & \text { if }\left\|\vec{\rho}^{\ell_{-, \gamma}}(x)-\vec{\rho}_{\beta}^{(\hat{p})}\right\|_{\star}<\zeta \\ 0 & \text { otherwise. }\end{cases}
$$




$$
\mathcal{M}_{\Lambda, \zeta}^{\hat{p}}:=\left\{\vec{\rho} \in L^{\infty}\left(R^{d}, S_{Q}\right): \eta_{x}^{\zeta}(\vec{\rho})=a_{\hat{p}} \quad \forall x \in \Lambda \sqcup \delta_{\text {out }}^{\gamma^{-1}}[\Lambda]\right\}
$$

and for any $\vec{\rho}^{*} \in \mathcal{M}_{\Lambda}^{\hat{p}}$, we define

$$
\mathcal{X}_{\Lambda, \vec{\rho}^{*}}^{\hat{p}}:=\left\{\vec{\rho} \in M_{\Lambda, \zeta}^{\hat{p}}: \vec{\rho}_{\Lambda^{c}}(x) \equiv \vec{\rho}_{\Lambda^{c}}^{*}(x)\right\}
$$

Theorem B.1. There are positive constant $\zeta_{0}, \omega, c_{\omega}$ so that for any $\zeta<\zeta_{0}, \gamma^{\alpha}<\kappa_{0} \zeta$ and any $\vec{\rho}^{*} \in M_{\Lambda, \zeta}^{\hat{p}}$, s.t.

- there is a unique $\vec{\psi} \in \mathcal{X}_{\Lambda, \vec{\rho}} *$ s.t.:

$$
\inf _{\vec{\rho} \in \mathcal{X}_{\Lambda, \vec{\rho}}^{\hat{p}} *} \mathcal{F}_{\gamma, u, \Lambda}\left(\vec{\rho} \mid \vec{\rho}_{\Lambda^{c}}^{*}\right)=\mathcal{F}_{\gamma, u, \Lambda}\left(\vec{\psi} \mid \vec{\rho}_{\Lambda^{c}}^{*}\right)
$$

- $\vec{\psi}$ is the unique solution of the mean field equation and has the following properties:

$* \vec{\psi}_{\Lambda} \in C^{\infty}\left(\Lambda, M_{\Lambda,\left(1-\kappa_{0}\right) \zeta}^{\hat{p}}\right), \quad \sup _{r \in \Lambda}\left\|\nabla \psi_{\Lambda}(r)\right\|_{\star} \leq \beta\left\|\nabla J_{\gamma}\right\|_{1}$

$*$

$$
\left\|\vec{\psi}_{\Lambda}(r)-\vec{\rho}^{\hat{p}}\right\| \leq c_{\omega} e^{-\omega \operatorname{dist}\left(r, \Lambda_{\neq}^{c}\right)}
$$

where $\Lambda_{\neq}^{c}:=\left\{r \in \Lambda^{c}: \operatorname{dist}(r, \Lambda) \leq \gamma^{-1} ; \vec{\rho}^{*}(r) \neq \vec{\rho}^{\hat{p}}\right\}$

- If $\vec{\psi}, \vec{\phi}$ are minimizers resp. in $\mathcal{X}_{\Lambda, \vec{\rho}_{1}}^{\hat{p}}, \mathcal{X}_{\Lambda, \vec{\rho}_{2}}^{\hat{p}}$ then:

$$
\left\|\vec{\psi}_{\Lambda}(r)-\vec{\phi}_{\Lambda}(r)\right\| \leq c_{\omega} e^{-\omega \operatorname{dist}\left(r, \Lambda_{1,2, \neq}^{c}\right)}
$$

where $\Lambda_{1,2, \neq}^{c}:=\left\{r \in \Lambda^{c}: \operatorname{dist}(r, \Lambda) \leq \gamma^{-1} ; \vec{\rho}_{1}(r) \neq \vec{\rho}_{2}(r)\right\}$

The proof of Theorem B.1 is obtained by defining a dynamic $\vec{T}_{t}^{u, \Lambda, \hat{p}}$ on $L^{\infty}\left(\mathbb{R}^{d}, S_{Q}\right)$, and proving that this dynamic maps $M_{\Lambda, \zeta}^{\hat{p}}$ into itself and that it is dissipative for the free energy $\mathcal{F}_{\gamma, u, \Lambda}$. The minimizer $\vec{\psi}$ is then obtained as the limit point of the orbit $\vec{T}_{t}^{u, \Lambda, \hat{p}}$ as $t \rightarrow \infty$.

Following 26] we define an opportune dynamic $\vec{T}_{t}^{u, \Lambda, \hat{p}}$ (suitable for our model) that has the properties that allow to conclude as in reference [26].

The essential point in the proof of the Theorem B.1 is the contraction property of the map $\overrightarrow{\mathfrak{M}}^{(u, \hat{p})}(\cdot)$ parameterized by $u, u \in[0,1]$, defined as follows:

$$
\begin{aligned}
& \mathfrak{M}_{q}^{(u, \hat{p})}(\vec{\rho})(r):=\frac{e^{\beta \mathcal{L}_{q}^{u, \hat{p}}(\vec{\rho} ; r)}}{\sum_{q^{\prime}} e^{\beta \mathcal{L}_{q^{\prime}}(\vec{\rho} ; r)}} \\
& \mathcal{L}_{q}^{u, \hat{p}}(\vec{\rho} ; r):=u \int d r^{\prime} J_{\gamma}\left(r, r^{\prime}\right) \rho_{q}\left(r^{\prime}\right)+(1-u) \rho_{q}^{\hat{p}}
\end{aligned}
$$

We state here a lemma which proof is postponed at the end of this appendix : 
Lemma B.2. There are $\zeta_{0}^{\prime}$ and $\kappa_{0}$ positive, so that for any $\zeta<\zeta_{0}^{\prime}$ and $\gamma^{\alpha}<\kappa_{0} \zeta$, any

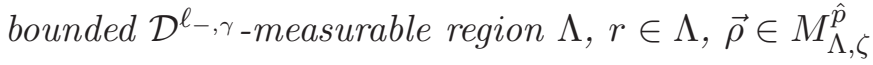

$$
\begin{aligned}
& \sup _{r \in \Lambda}\left\|\overrightarrow{\mathcal{L}}^{u, \hat{p}}(\vec{\rho})(r)-\vec{\rho}^{\hat{p}}(r)\right\|_{\star} \leq u\left(1+c_{d} \kappa_{0}\right) \zeta<2 \zeta \\
& \sup _{r \in \Lambda}\left\|\overrightarrow{\mathfrak{M}}^{(u, \hat{p})}(\vec{\rho})(r)-\vec{\rho}^{\hat{p}}(r)\right\|_{\star} \leq u\left(1-\kappa_{0}\right) \zeta
\end{aligned}
$$

We then define a dynamic given by the semigroup $\vec{T}_{t}^{u, \Lambda, \hat{p}}(\cdot), t \geq 0$ on $L^{\infty}\left(\Lambda, S_{Q}\right)$,

$$
\vec{T}_{t}^{u, \Lambda, \hat{p}}(\vec{\rho}):=\left(T_{1, t}^{u, \Lambda, \hat{p}}\left(\rho_{1}\right), \ldots, T_{Q, t}^{u, \Lambda, \hat{p}}\left(\rho_{Q}\right)\right) \quad \text { for any } \vec{\rho} \in L^{\infty}\left(\mathbb{R}^{d}, S_{Q}\right)
$$
where $T_{q, t}^{u, \Lambda, \hat{p}}\left(\rho_{i}^{*}\right)$ are solutions of the Cauchy problem:

$$
\left\{\begin{array}{lr}
\frac{d \rho_{q}^{\Lambda}(r, t)}{d t}=-\rho_{q}^{\Lambda}(r, t)+\mathfrak{M}_{q}^{(u, \hat{p})}\left(\vec{\rho}^{\Lambda}\right) & \text { for any } q=1, \ldots, Q \\
\vec{\rho}^{\Lambda}(r, t)=\vec{\rho}^{*}(r, t) & (r, t) \in\left[\Lambda^{c} \times\{t \geq 0\}\right] \sqcup\left[\mathbb{R}^{d} \times\{t=0\}\right]
\end{array}\right.
$$

Existence, uniqueness and continuity w.r.t. the initial datum of the solution follows by the continuity and the Lipschitz property of the r.h.s. of equation (B.5)

Notice that $\vec{T}_{t}^{u, \Lambda}(\cdot)$ maps $L^{\infty}\left(\mathbb{R}^{d}, S_{Q}\right)$ in itself, and has as a fixed point $\vec{\rho}^{\hat{p}}$. We next prove the following properties:

(1) $\vec{T}_{t}^{u, \Lambda, \hat{p}}\left(M_{\Lambda, \zeta}^{\hat{p}}\right) \sqsubset M_{\Lambda, \zeta}^{\hat{p}}$ for any $t \geq 0$

(2) For any $\vec{\rho}_{0} \in L^{\infty}\left(\mathbb{R}^{d}, S_{Q}\right)$ and $\Lambda$ a Borel set, $T_{q, t}^{u, \Lambda, \hat{p}}\left(\vec{\rho}_{0}\right), q=1, \ldots, Q$, converges by subsequences as $t \rightarrow \infty$ to functions $v_{q}$ that are bounded in $\Lambda$ and with $\nabla v_{q}$ bounded in $\Lambda$. The limit points are solutions of (B.6) below.

(3) $\mathcal{F}_{\gamma, u, \Lambda}\left(\vec{T}_{t}^{u, \Lambda, \hat{p}}\left(\vec{\rho}_{0}\right)\right)$ decrease with $t$, strictly unless $\vec{\rho}_{0}$ is stationary, in which case satisfies:

$$
\rho_{0, q}=\frac{e^{\beta \mathcal{L}_{q}^{u}\left(\vec{\rho}_{0}(r, t)\right)}}{\sum_{q^{\prime}=1}^{Q} e^{\beta \mathcal{L}_{q^{\prime}}^{u}\left(\vec{\rho}_{0}(r, t)\right)}} \quad \forall q=1, \ldots Q \quad \forall r \in \Lambda
$$

(4) As a consequence of the property (3), for any $\vec{\rho}^{*}(r) \in M_{\Lambda, \zeta}^{\hat{p}}$, the minimizers of $\mathcal{F}_{\gamma, u, \Lambda}(\cdot)$ in $\mathcal{X}_{\Lambda, \vec{\rho}^{*}}:=\left\{\vec{\rho} \in M_{\Lambda, \zeta}^{\hat{p}}: \vec{\rho}_{\Lambda^{c}}(r) \equiv \vec{\rho}_{\Lambda^{c}}^{*}(r)\right\}$ are solutions of (B.6): $\vec{\rho}=\overrightarrow{\mathfrak{M}}^{u, \hat{p}}(\vec{\rho})$. By the contraction property of the map $\overrightarrow{\mathfrak{M}}^{u, \hat{p}}(\cdot)$ we get uniqueness of the minimizer. 
(1) Clearly we have $\vec{T}_{t}^{u, \Lambda, \hat{p}}\left(S_{Q}\right) \sqsubset S_{Q}$. To prove the first point, let then $\tau>0$, $\vec{\rho}_{0} \in M_{\Lambda, \zeta}^{\hat{p}}$ and

$$
\mathcal{X}_{\tau, \vec{\rho}_{0}}:=\left\{\vec{\rho} \in L^{\infty}\left(\mathbb{R}^{d} \times[0, \tau] ; S_{Q}\right): \vec{\rho}(r, t)=\vec{\rho}_{0}(r) \quad(r, t) \in\left[\mathbb{R}^{d} \times 0\right] \sqcup\left[\Lambda^{c} \times[0, \tau]\right]\right\}
$$

Let $\hat{\Omega}^{\hat{p}}(\cdot)$ the map from $\mathcal{X}_{\tau, \vec{\rho}_{0}}$ into itself defined for any $t \in[0, \tau], r \in \Lambda$ as

$$
\vec{\Omega}^{\hat{p}}(\vec{\rho})(r, t)=e^{-t} \vec{\rho}_{0}(r)+\int_{0}^{t} e^{-(t-s)} \overrightarrow{\mathfrak{M}}^{(u, \hat{p})}(\vec{\rho}) d s
$$

if $\tau$ is small enough $\vec{\Omega}^{\hat{p}}$ is a contraction and its fixed point is the solution of (B.5), $T_{t}^{u, \Lambda, \hat{p}}\left(\vec{\rho}_{0}\right), t \in[0, \tau]$. By (B.3) the set:

$$
\left\{\vec{v} \in \mathcal{X}_{\tau, \vec{\rho}_{0}}: \vec{v}(\cdot, t) \in M_{\Lambda, \zeta}^{\hat{p}} \forall t \in[0, \tau]\right\}
$$

is invariant under the map $\hat{\Omega}$, and since it is closed, it contains the fixed point of $\hat{\Omega}$. By induction the statement can be extended fo any $t: T_{t}^{u, \Lambda, \hat{p}}\left(\vec{\rho}_{0}\right) \sqsubset M_{\Lambda, \zeta}^{\hat{p}}$, $t \geq 0$.

(2) Convergence on subsequences follows by Ascoli-Arzelà theorem, after having written the integral expression of the evolution (B.5) and observed $T_{q, t}^{u, \Lambda, \hat{p}}(\vec{\rho})-e^{-t} \rho_{q}$ is bounded with bounded gradient.

(3) The decreasing of the free energy functional, follows by observing that:

$$
\frac{d}{d t} \mathcal{F}_{\gamma, u, \Lambda}\left(\left[\vec{T}_{t}^{u, \Lambda, \hat{p}}(\vec{\rho})\right] \mid \vec{\rho}_{\Lambda^{c}}\right)<0
$$

an explicit calculation gives in fact :

$$
\begin{gathered}
\frac{d}{d t} \mathcal{F}_{\gamma, u, \Lambda}\left(\left[\vec{T}_{t}^{u, \Lambda, \hat{p}}(\vec{\rho})\right] \mid \vec{\rho}_{\Lambda^{c}}=\sum_{q} \int_{\Lambda} d r\left(-\mathcal{L}_{q}^{u}\left(\vec{T}_{t}^{u, \Lambda, \hat{p}}(\vec{\rho})\right)+\frac{1}{\beta}\left(1+\ln T_{q, t}^{u, \Lambda, \hat{p}}(\vec{\rho})\right)\right)\right. \\
\cdot\left(-T_{q, t}^{u, \Lambda, \hat{p}}(\vec{\rho})+\mathfrak{M}_{q}^{(u, \hat{p})}\left(\vec{T}_{t}^{u, \Lambda, \hat{p}}(\vec{\rho})\right)\right) \\
\left.=\sum_{q} \int_{\Lambda} d r \frac{1}{\beta}\left(\ln \frac{T_{q, t}^{u, \Lambda, \hat{p}}(\vec{\rho})}{\mathfrak{M}_{q}^{(u, \hat{p})}\left(\vec{T}_{t}^{u, \Lambda, \hat{p}}(\vec{\rho})\right)}+1-\ln \sum_{q^{\prime}} e^{\beta \mathcal{L}_{q^{\prime}}^{u}\left(\vec{T}_{t}^{u, \Lambda, \hat{p}}(\vec{\rho})\right)}\right)\right) \\
\cdot\left(-T_{q, t}^{u, \Lambda, \hat{p}}(\vec{\rho})+\mathfrak{M}_{q}^{(u, \hat{p})}\left(\vec{T}_{t}^{u, \Lambda, \hat{p}}(\vec{\rho})\right)\right) \\
=\frac{1}{\beta} \sum_{q} \int_{\Lambda} d r\left(\ln \frac{T_{q, t}^{u, \Lambda, \hat{p}}(\vec{\rho})}{\mathfrak{M}_{q}^{(u, \hat{p})}\left(\vec{T}_{t}^{u, \Lambda}(\vec{\rho})\right)}\right)\left(-T_{q, t}^{u, \Lambda, \hat{p}}(\vec{\rho})+\mathfrak{M}_{q}^{(u, \hat{p})}\left(\vec{T}_{t}^{u, \Lambda, \hat{p}}(\vec{\rho})\right)\right) \\
+\frac{K}{\beta} \sum_{q} \int_{\Lambda} d r\left(-T_{q, t}^{u, \Lambda, \hat{p}}(\vec{\rho})+\mathfrak{M}_{q}^{(u, \hat{p})}\left(\vec{T}_{t}^{u, \Lambda, \hat{p}}(\vec{\rho})\right)\right)
\end{gathered}
$$

with $K=\left(1-\ln \sum_{q^{\prime}} e^{\beta \mathcal{L}_{q^{\prime}}^{u}(\vec{\rho})}\right)$. By normalization condition, last term is null, while the first one is negative (in fact if the first factor inside the integral is positive, 
the last one is negative and viceversa). We denote by:

$$
\begin{gathered}
\mathcal{D}_{\Lambda}^{u, \hat{p}}(\vec{\rho}):=\frac{1}{\beta} \sum_{i}\left(\ln \frac{\rho_{i}}{\mathfrak{M}_{i}^{(u, \hat{p})}(\vec{\rho})}\right)\left(-\rho_{i}+\mathfrak{M}_{i}^{(u, \hat{p})}(\vec{\rho})\right) \\
\mathcal{D}_{\Lambda}^{u, \hat{p}}(\vec{\rho})=0 \text { if and only if } \vec{\rho} \text { satisfies the equation: } \\
\rho_{q}=\mathfrak{M}_{q}^{(u, \hat{p})}(\vec{\rho}) \quad \forall q=1, \ldots, Q
\end{gathered}
$$

To conclude we need a lower bound on $\vec{T}_{t}^{u, \Lambda, \hat{p}}(\vec{\rho})$ that assure that the dynamic is always well defined for any time $t>0$. (notice that $\mathcal{D}_{\Lambda}^{u, \hat{p}}(\vec{\rho})$ diverges if one of the coordinates $\rho_{q}(t)$ becomes null.)

Lemma B.3. Let $\vec{\rho} \in L^{\infty}\left(\mathbb{R}^{d}, S_{Q}\right), \Lambda$ a Borel set in $\mathbb{R}^{d}, \vec{\rho}^{\Lambda}(\cdot, t)=\vec{T}_{t}^{u, \Lambda, \hat{p}}(m)$ and $v_{q}^{0}:=\inf _{r^{\prime} \in \Lambda} \rho_{q}^{\Lambda}\left(r^{\prime}\right)$. Then for all $r \in \Lambda$ and $t \geq 0$ :

$$
\rho_{q}(r, t) \geq\left[v_{q}^{0}-c_{q}(u, \beta)\right] e^{-t}+c_{q}(u, \beta)
$$

where $c_{q}(u, \beta)=\frac{1}{e^{\beta u}} \frac{e^{(1-u) \beta \rho_{q}^{\hat{p}}}}{\sum_{q^{\prime}} e^{(1-u) \beta \rho_{q^{\prime}}^{\hat{p}}}} \geq \frac{1}{Q e^{\beta[u+(1-u)]}} \geq \frac{1}{Q e^{\beta}}$

Proof. For any $q=1, \ldots, Q$, let $v_{q}(t)$ the solutions of the Cauchy problems:

$$
\left\{\begin{array}{l}
\frac{d v_{q}(t)}{d t}=-v_{q}(t)+\frac{1}{e^{\beta u}} c_{q}(u, \beta) \\
\vec{v}(t)=\vec{v}^{0}
\end{array}\right.
$$

Let $w_{q}(r, t):=v_{q}(t) \mathbf{1}_{r \in \Lambda}+\vec{\rho}^{*}(r, t) \mathbf{1}_{r \in \Lambda^{c}}$, Since $-w_{q}(r, t)+\frac{1}{e^{\beta u}} c_{q}(u, \beta) \leq-w_{q}(r, t)+$ $\mathfrak{M}_{q}^{(u, \hat{p})}(\vec{w})$ uniformly in $r$ and $w_{q} \in[0,1]$. By Gronwall Lemma, $\rho_{q}(r, t) \geq w_{q}(r, t)$ for any $r \in \mathbb{R}^{d}, t>0$, and it is strictly positive for any $t>s>0$.

By Lemma B.3 for any $s>0$, the functions $\left[T_{q, t}^{u, \Lambda, \hat{p}}(\vec{\rho})\right]_{\Lambda}, t \geq s$, for any $q \in[1, \ldots, Q]$ are bounded away from 0 , so that:

$$
\mathcal{F}_{\gamma, u, \Lambda}\left(\left[\vec{T}_{t}^{u, \Lambda, \hat{p}}(\vec{\rho})\right]_{\Lambda} \mid \vec{\rho}^{*}\right)-\mathcal{F}_{\gamma, u, \Lambda}\left(\left[\vec{T}_{s}^{u, \Lambda, \hat{p}}(\vec{\rho})\right]_{\Lambda} \mid \vec{\rho}^{*}\right)=\int_{s}^{t} \mathcal{D}_{\Lambda}^{u, \hat{p}}\left(\vec{T}_{t^{\prime}}^{u, \Lambda, \hat{p}}(\vec{\rho})\right) d t^{\prime}
$$

Since $\mathcal{D}_{\Lambda}^{u, \hat{p}}\left(\vec{T}_{s}^{u, \Lambda, \hat{p}}(\vec{\rho})\right)$ is monotone in $s$, by the Lebesgue dominated convergence theorem, the limit $s \rightarrow 0$ exists, and we get:

$$
\mathcal{F}_{\gamma, u, \Lambda}\left(\left[\vec{T}_{t}^{u, \Lambda, \hat{p}}(\vec{\rho})\right]_{\Lambda} \mid \vec{\rho}^{*}\right)-\mathcal{F}_{\gamma, u, \Lambda}\left(\vec{\rho}_{\Lambda} \mid \vec{\rho}^{*}\right)=\int_{0}^{t} \mathcal{D}_{\Lambda}^{u, \hat{p}}\left(\vec{T}_{t^{\prime}}^{u, \Lambda, \hat{p}}(\vec{\rho})\right) d t^{\prime}
$$

We omit the proof of the following theorem that follows by previous analysis: 
Theorem B.4. Let $\vec{\rho} \in L^{\infty}\left(\mathbb{R}^{d}, S_{Q}\right)$ and $\Lambda$ a bounded, Borel set. Then, any limit point of $\vec{T}_{t}^{\Lambda}(\vec{\rho})$ satisfies (B.6) and for any $\vec{\rho}^{*} \in L^{\infty}\left(\Lambda^{c}, S_{Q}\right)$ there is $\vec{v} \in C^{1}\left(\Lambda, S_{Q}\right)$, with $\nabla v_{x}$ bounded for any $x$ in $\Lambda$ s.t.

$$
\mathcal{F}_{\gamma, u, \Lambda}\left(\vec{v} \mid \vec{\rho}_{\Lambda^{c}}^{*}\right) \leq \mathcal{F}_{\gamma, u, \Lambda}\left(\vec{\rho}_{\Lambda}^{*} \mid \vec{\rho}_{\Lambda^{c}}^{*}\right)
$$

As a corollary of the Theorem B.1 we have the following result used in Subsection 7.2 and 8

Corollary B.5. There are positive constants $\omega$ and $c_{\omega}^{\prime}$ so that for any $u \in[0,1]$ and any $\vec{\rho}^{*} \in M_{\Lambda, \zeta, \ell}^{\hat{p}}$ there is $\vec{\psi}^{(u)} \in \mathcal{X}_{\Lambda, \vec{\rho}} *$ with the following properties:

$$
\begin{array}{lr}
\vec{\psi}^{(u)}(r)=\vec{\rho}^{*}(r) & r \in \Lambda^{c} \sqcup \delta_{\text {in }}^{\gamma^{-1}}[\Lambda] \\
\vec{\psi}^{(u)}(r)=\vec{\rho}^{\hat{p}}(r) & r \in \Lambda \backslash \delta_{\text {in }}^{\ell_{+}, \gamma / 4}[\Lambda] \\
\mathcal{F}_{\gamma, u, \lambda}\left(\vec{\psi}^{(u)}\right) \leq \mathcal{F}_{\gamma, u, \lambda}\left(\vec{\rho}^{*}\right)+c_{\omega}|\Lambda| e^{-\omega \ell_{+, \gamma} / 4} &
\end{array}
$$

We conclude this appendix by giving the proof of the Lemma B.2

Proof of Lemma B.2. In order to prove the first statement, we define a $\mathcal{D}^{\left(\ell_{-, \gamma}\right)}$ - measurable approximation of the interaction kernel as:

$$
J_{\gamma}^{\left(\ell_{-, \gamma}\right)}(x,\langle y\rangle)=\frac{1}{\left|C^{\left(\ell_{-, \gamma}\right)}\right|} \int_{y^{\prime} \in C_{y}^{\left(\ell_{-, \gamma}\right)}} J_{\gamma}\left(x, y^{\prime}\right) d y^{\prime}
$$

We have for $\ell_{-, \gamma}=\gamma^{-1+\alpha} \ll \gamma^{-1}$,

$$
\begin{aligned}
\left|J_{\gamma}^{\left(\ell_{-, \gamma}\right)}(x,\langle y\rangle)-J_{\gamma}(x, y)\right| \leq \sup _{y^{\prime} \in C_{y}^{\left(\ell_{-, \gamma}\right)}}\left|J_{\gamma}^{\left(\ell_{-, \gamma}\right)}(x,\langle y\rangle)-J_{\gamma}\left(x, y^{\prime}\right)\right| \\
\leq \sqrt{d} \ell_{-, \gamma} \gamma^{d+1}\|\nabla J\|_{\infty} \mathbf{1}_{\{|x-y|\} \leq 2 \gamma^{-1}}
\end{aligned}
$$

Using this result, we can write for all $r$ and all $q$,

$$
\begin{aligned}
& \left|\mathcal{L}_{q}^{u}(\vec{\rho})(r)-\rho_{q}^{\hat{p}}\right|=u\left|\int d r^{\prime} J_{\gamma}\left(r, r^{\prime}\right)\left(\rho_{q}\left(r^{\prime}\right)-\rho_{q}^{\hat{p}}\right)\right| \\
& \leq u\left|\int d r^{\prime} J_{\gamma}^{\left(\ell_{-, \gamma}\right)}\left(r,\left\langle r^{\prime}\right\rangle\right)\left(\rho_{q}\left(r^{\prime}\right)-\rho_{q}^{\hat{p}}\right)\right|+u\left|\int d r^{\prime}\left(J_{\gamma}\left(r, r^{\prime}\right)-J_{\gamma}^{\left(\ell_{-, \gamma}\right)}\left(r,\left\langle r^{\prime}\right\rangle\right)\right)\left(\rho_{q}\left(r^{\prime}\right)-\rho_{q}^{\hat{p}}\right)\right| \\
& \leq u\left(\sum_{j \in\left(\ell_{-, \gamma} \mathbb{Z}\right)^{d}} J_{\gamma}^{\left(\ell_{-, \gamma}\right)}(r,\langle x\rangle) \int_{r^{\prime} \in C_{j}^{\left(\ell_{-, \gamma}\right)}}\left|\rho_{q}\left(r^{\prime}\right)-\rho_{q}^{\hat{p}}\right| d r^{\prime}+2^{d} \sqrt{d}\|\nabla J\|_{\infty} \gamma \ell_{-, \gamma}\right) \\
& \leq u\left(1+c_{d} \kappa_{0}\right) \zeta
\end{aligned}
$$

for $\gamma^{\alpha} \leq \kappa_{0} \zeta$ and $c_{d}=2^{d} \sqrt{d}\|\nabla J\|_{\infty}$. Hence

$$
\sup _{r}\left\|\overrightarrow{\mathcal{L}}^{u}(\vec{\rho})-\vec{\rho} \hat{p}\right\|_{\star} \leq u\left(1+c_{d} \kappa_{0}\right) \zeta \leq 2 \zeta
$$

for $\kappa_{0}$ small enough. 
In order to prove (B.3), we take $\zeta_{0}^{\prime}$ small enough so that theorem A.3 holds ( for example $\left.\zeta_{0}^{\prime}<\left(\frac{1}{2 \beta Q}\right)^{2}\right)$ for all $\zeta<\zeta_{0}^{\prime}$. We get for any $r \in \Lambda$

$$
\begin{aligned}
\left\|\overrightarrow{\mathfrak{M}}^{(u, \hat{p})}(\vec{\rho})(r)-\vec{\rho} \hat{p}(r)\right\|_{\star} & =\left\|\vec{g}\left(\overrightarrow{\mathcal{L}}^{u}(\vec{\rho})\right)(r)-\vec{g}(\vec{\rho} \hat{p})(r)\right\|_{\star} \\
& \leq\left(1-\frac{1}{2 Q}\right)\left\|\overrightarrow{\mathcal{L}}^{u}(\vec{\rho})(r)-\vec{\rho}{ }^{\hat{p}}(r)\right\|_{\star} \\
& \leq\left(1-\frac{1}{2 Q}\right) u\left(1+c_{d} \kappa_{0}\right) \zeta \leq u\left(1-\kappa_{0}\right) \zeta
\end{aligned}
$$

having chosen $\kappa_{0}$ so small that

$$
\kappa_{0} \leq \frac{1}{2 Q\left(1+c_{d}\right)}
$$

and (B.3) holds.

\section{Appendix C. Existence of the pressures $P_{\mathrm{abs}, \gamma, \beta}^{ \pm}$of the abstract models}

Let $\left\{\Lambda_{n}\right\}$ a sequence of sets of side $2^{n} \ell_{+, \gamma}$ and

$$
D_{\gamma, \beta}(n):=\frac{\ln Z_{\mathrm{abs}, \Lambda_{n} \gamma, \beta}^{ \pm}\left(\vec{\rho}_{\beta}^{ \pm}\right)}{\beta\left|\Lambda_{n}\right|}-\frac{\ln Z_{\mathrm{abs}, \Lambda_{n-1}, \gamma, \beta}^{ \pm}\left(\vec{\rho}_{\beta}^{ \pm}\right)}{\beta\left|\Lambda_{n-1}\right|}
$$

The proof of existence and continuity in $\beta$ o the abstract pressures follows by the continuity in $\beta$ of $D_{\gamma, \beta}(n)$ and by proving that there is a constant $\kappa_{7}$ :

$$
\left|D_{\gamma, \beta}(n)\right| \leq \kappa_{7} 2^{-n}
$$

Proof. Decomposing $\Lambda_{n}$ into cubes $\Lambda_{n-1}(k), k=1, \ldots, 2^{d}$, since the interaction energy is bounded uniformly in $\vec{\xi}$ and recalling (6.6), we have:

$$
\begin{aligned}
& \ln Z_{\mathrm{abs}, \Lambda_{n} \gamma, \beta}^{ \pm}\left(\vec{\rho}_{\beta}^{ \pm}\right) \geq \\
& \quad 2^{d} \ln Z_{\mathrm{abs}, \Lambda_{n-1} \gamma, \beta}^{ \pm}\left(\vec{\rho}_{\beta}^{ \pm}\right)-c \gamma^{-1}\left(2^{n} \ell_{+, \gamma}\right)^{d-1}-2 d\left(2^{n-1}\right)^{d-1} e^{-\frac{\mathfrak{K}_{\gamma}}{2}}
\end{aligned}
$$

where, denoting by $\left[\delta \Lambda_{n}^{0}\right]:=\sqcup_{k=1}^{2^{d}} \delta_{i n}^{\ell+, \gamma}\left[\Lambda_{n-1}(k)\right]$, we have used the estimate

$$
\left|\sum_{\Delta \sqsubset \Lambda_{n}} U_{\Delta}(\vec{\xi})-\sum_{k} \sum_{\Delta \sqsubset \Lambda_{n-1}(k)} U_{\Delta}(\vec{\xi})\right|=\sum_{\Delta: \Delta \sqcap\left[\delta \Lambda_{n}^{0}\right] \neq \emptyset}\left\|U_{\Delta}(\vec{\xi})\right\|_{\infty} \leq 2 d\left(2^{n-1}\right)^{d-1} e^{-\frac{\mathfrak{K}_{\gamma}}{2}}
$$

(C.2) gives:

$$
D_{\gamma, \beta}(n) \geq-c 2^{-n}
$$


the same arguments give also the upper bound:

$$
D_{\gamma, \beta}(n) \leq-c 2^{-n}
$$

\section{Appendix D. Existence of the pressure $P_{\mathrm{abs}, \gamma ; 0}^{ \pm}$and surface correction.}

In this appendix we prove the following Lemma:

Lemma D.1. There exists a constant $c_{d}$ such that for $\gamma$ small enough

$$
\left|P_{\mathrm{abs}, \gamma ; 0}^{ \pm}+\phi_{\beta}^{\mathrm{mf}}\left(\rho^{ \pm}\right)\right| \leq c_{d} \gamma
$$

where $P_{\mathrm{abs}, \gamma ; 0}^{ \pm}$is defined in (4.16) for $u=0$, and

$$
R_{\mathrm{abs}, \Lambda ; 0}^{ \pm}=R_{\gamma, \Lambda}^{\mathrm{mf}}:=\frac{\beta}{2} \sum_{\substack{x \in \Lambda \\ y \in \Lambda^{c}}} J_{\gamma}(x, y)\left(\vec{\rho}^{ \pm} \cdot \vec{\rho}^{ \pm}\right)
$$

ProOF.

We denote by $C_{0} \equiv C_{0}^{\ell_{+, \gamma}}$, the cube of the partition $\mathcal{D}^{\ell_{+, \gamma}}$ that contains the point 0 ,

$$
\begin{aligned}
\ln Z_{\mathrm{abs}, \beta, \Lambda ; 0}^{ \pm} & =\ln \left\{\sum_{\vec{\xi}_{\Lambda}} \prod_{x \in \Lambda} e^{\beta\left(\left(\vec{\xi}_{x}-\rho^{ \pm}\right) \cdot \overrightarrow{\mathcal{L}}\left(\rho^{ \pm}\right)\right)} \mathbf{1}_{\vec{\xi} \in \mathcal{X}_{C_{0}}^{ \pm}}\right\}-\beta H_{\gamma, \Lambda}\left(\vec{\rho}_{\Lambda}^{ \pm} \mid \vec{\rho}_{\Lambda^{c}}^{ \pm}\right) \\
= & \ln \left\{\sum_{\vec{\xi}_{\Lambda}} \prod_{x \in \Lambda} e^{\beta\left(\vec{\xi}_{x} \cdot \overrightarrow{\mathcal{L}}\left(\rho^{ \pm}\right)\right)} \mathbf{1}_{\vec{\xi} \in \mathcal{X}_{C_{0}}^{ \pm}}\right\}-\beta|\Lambda| \mathfrak{J}_{\gamma}\left(\rho^{ \pm} \cdot \vec{\rho}^{ \pm}\right)-\beta H_{\gamma, \Lambda}\left(\vec{\rho}_{\Lambda}^{ \pm} \mid \rho_{\Lambda^{c}}^{ \pm}\right) \\
= & \frac{|\Lambda|}{\left|C_{0}\right|} \ln \left\{\sum_{\vec{\xi}_{C_{0}}} \prod_{x \in C_{0}} e^{\beta\left(\vec{\xi}_{x} \cdot \overrightarrow{\mathcal{L}}\left(\rho^{ \pm}\right)\right)} \mathbf{1}_{\vec{\xi} \in \mathcal{X}_{C_{0}}^{ \pm}}\right\}-\frac{\beta|\Lambda|}{2} \mathfrak{J}_{\gamma}\left(\rho^{ \pm} \cdot \vec{\rho}^{ \pm}\right) \\
& +\frac{\beta \mid}{2} \sum_{\substack{x \in \Lambda \\
y \in \Lambda^{c}}} J_{\gamma}(x, y)\left(\vec{\rho}^{ \pm} \cdot \vec{\rho}^{ \pm}\right)
\end{aligned}
$$

where we recall $H_{\gamma, \Lambda}\left(\vec{\rho}_{\Lambda}^{ \pm} \mid \vec{\rho}_{\Lambda^{c}}^{ \pm}\right)=-\frac{1}{2} \sum_{x \in \Lambda} J_{\gamma}(x, y)\left(\vec{\rho}^{ \pm} \cdot \vec{\rho}^{ \pm}\right)-\sum_{y \in \Lambda} \underset{y \in \Lambda}{ } J_{\gamma}(x, y)\left(\vec{\rho}^{ \pm} \cdot \vec{\rho}^{ \pm}\right)=$ $-\frac{1}{2} \sum_{\substack{x \in \Lambda \\ y \in \mathbb{Z}^{d}}} J_{\gamma}(x, y)\left(\vec{\rho}^{ \pm} \cdot \vec{\rho}^{ \pm}\right)-\frac{1}{2} \sum_{\substack{x \in \Lambda \\ y \in \Lambda^{c}}} J_{\gamma}(x, y)\left(\vec{\rho}^{ \pm} \cdot \vec{\rho}^{ \pm}\right)$. Then we get:

$$
\begin{aligned}
P_{\mathrm{abs}, \gamma, 0}^{ \pm} & :=\lim _{\Lambda \nearrow \infty} \frac{1}{\beta|\Lambda|} \ln Z_{\mathrm{abs}, \beta, \Lambda ; 0}^{ \pm}=\lim _{\Lambda \nearrow \infty} \frac{1}{\beta|\Lambda|} \ln \left\{\sum_{\vec{\xi}_{C_{0}}} \prod_{x \in C_{0}} e^{-\beta \mathfrak{H}_{\gamma, \Lambda}^{ \pm}(\vec{\xi})} \mathbf{1}_{\vec{\xi} \in \mathcal{X}_{C_{0}}^{ \pm}}\right\} \\
& \equiv \frac{1}{\beta\left|C_{0}\right|} \ln \left\{\sum_{\vec{\xi}_{C_{0}}} \prod_{x \in C_{0}} e^{\beta\left(\vec{\xi} \cdot \overrightarrow{\mathcal{L}}\left(\rho^{ \pm}\right)\right)} \mathbf{1}_{\vec{\xi} \in \mathcal{X}_{C_{0}}^{ \pm}}\right\}-\frac{1}{2} \mathfrak{J}_{\gamma}\left(\vec{\rho}^{ \pm} \cdot \vec{\rho}^{ \pm}\right)
\end{aligned}
$$


A comparison of (D.3) and (D.4) gives directly (D.2).

We now prove (D.1)

Let consider the first term of (D.4):

$$
\begin{aligned}
\frac{1}{\beta\left|C_{0}\right|} \ln \left\{\sum_{\vec{\xi}_{C_{0}}} \prod_{x \in C_{0}} e^{\beta\left(\vec{\xi} \cdot \overrightarrow{\mathcal{L}}\left(\rho^{ \pm}\right)\right)} \mathbf{1}_{\vec{\xi} \in \mathcal{X}_{C_{0}}^{ \pm}}\right\} \\
=\frac{1}{\beta\left|C_{0}\right|} \ln G_{ \pm, \gamma ; 0}\left[\mathbf{1}_{\vec{\xi} \in \mathcal{X}_{C_{0}}^{ \pm}}\right]+\frac{1}{\beta\left|C_{0}\right|} \ln \sum_{\vec{\xi}_{C_{0}}} \prod_{x \in C_{0}} e^{\beta\left(\vec{\xi}_{x} \cdot \overrightarrow{\mathcal{L}}\left(\rho^{ \pm}\right)\right)} \\
=\frac{1}{\beta\left|C_{0}\right|} \ln G_{ \pm, \gamma ; 0}\left[\mathbf{1}_{\vec{\xi} \in \mathcal{X}_{C_{0}}^{ \pm}}\right]+\frac{1}{\beta} \ln \sum_{i=1, Q} e^{\beta \mathfrak{J}_{\gamma} \rho_{i}^{ \pm}}
\end{aligned}
$$

where $G_{ \pm, \gamma ; 0}\left[\mathbf{1}_{\vec{\xi} \in \mathcal{X}_{C_{0}}^{ \pm}}\right]$is the probability of the event $\mathbf{1}_{\vec{\xi} \in \mathcal{X}_{C_{0}}^{ \pm}}$w.r.t. the Gibbs measure specified by:

$$
\mu_{\gamma ; 0}^{ \pm}:=\frac{e^{\beta \sum_{x}\left(\vec{\xi}_{x} \cdot \overrightarrow{\mathcal{L}}\left(\rho^{ \pm}\right)\right)}}{Z_{\gamma ; 0}^{ \pm}}
$$

Postponing the proof that the first term is negligible, as

$$
G_{ \pm, \gamma ; 0}\left[\mathbf{1}_{\vec{\xi} \in \mathcal{X}_{C_{0}}^{ \pm}}\right]>1-C e^{-c \gamma^{2 a}\left|C^{\ell-, \gamma}\right|}
$$

we consider the second term. We have

$$
\left|\frac{1}{\beta} \ln \sum_{i=1, Q} e^{\beta \mathfrak{J}_{\gamma} \rho_{i}^{ \pm}}-\frac{1}{\beta} \ln \sum_{i=1, Q} e^{\beta \rho_{i}^{ \pm}}\right| \leq 2^{d} \sqrt{d}\|\nabla \mathcal{J}\|_{\infty} \gamma
$$

and

$$
\begin{aligned}
\frac{1}{\beta} \ln \sum_{i=1, Q} e^{\beta \rho_{i}^{ \pm}} & =\frac{1}{\beta} \sum_{j} \rho_{j}^{ \pm} \ln \sum_{i=1, Q} e^{\beta \rho_{i}^{ \pm}} \\
& =\frac{1}{\beta} \sum_{j} \rho_{j}^{ \pm}\left[-\ln \rho_{j}^{ \pm}+\beta \rho_{j}^{ \pm}\right]=-\phi_{\beta}^{\mathrm{mf}}\left(\vec{\rho}^{ \pm}\right)
\end{aligned}
$$

Going back to (D.4), and using (D.5), we get

$$
\left|P_{\mathrm{abs}, \gamma, 0}^{ \pm}+\phi_{\beta}^{\mathrm{mf}}\left(\vec{\rho}^{ \pm}\right)\right| \leq c_{d} \gamma+C e^{-c \gamma^{2 a}\left|C^{\ell-, \gamma}\right|} \leq c_{d}^{\prime} \gamma
$$

for $\gamma$ small enough.

To conclude we are then left with the proof of (D.5). Since the derivation is similar (and simpler) to what is done in section 8 but with a different free energy functional, we just sketch the proof here. We consider the one body functional $\mathcal{F}_{\beta, C_{0}}^{(1)}$ on $C_{0}$,

$$
\mathcal{F}_{\beta, C_{0}}^{(1)}\left(\vec{\rho}_{C_{0}}\right)=-\frac{1}{2} \int_{C_{0}}\left(\vec{\rho}^{ \pm} \cdot \vec{\rho}_{C_{0}}(x)\right) d x-\frac{1}{\beta} \int_{C_{0}} I\left(\vec{\rho}_{C_{0}}(x)\right) d x
$$


Using a result similar to 5.1 leads to an estimate valid for $\gamma$ small enough of $G_{ \pm, \gamma ; 0}\left[\mathbf{1}_{\vec{\xi} \notin \mathcal{X}_{C_{0}}^{ \pm}}\right]$ in terms of the functional $\mathcal{F}_{\beta, C_{0}}^{(1)}$, as

$$
\begin{aligned}
G_{ \pm, \gamma ; 0}\left[\mathbf{1}_{\vec{\xi} \notin \mathcal{X}_{C_{0}}^{ \pm}}\right] & =\frac{\sum_{\vec{\xi}_{C_{0}}} \prod_{x \in C_{0}} e^{\beta\left(\vec{\xi}_{x} \cdot \overrightarrow{\mathcal{L}}\left(\rho^{ \pm}\right)\right)} \mathbf{1}_{\vec{\xi} \notin \mathcal{X}_{C_{0}^{+}}^{ \pm}}}{\sum_{\vec{\xi}_{C_{0}}} \prod_{x \in C_{0}} e^{\beta\left(\vec{\xi}_{x} \cdot \overrightarrow{\mathcal{L}}\left(\rho^{ \pm}\right)\right)}} \\
& =\frac{\sum_{\vec{\xi}_{C_{0}}} \prod_{x \in C_{0}} e^{\beta \mathfrak{J}_{\gamma}\left(\vec{\xi}_{x} \cdot \vec{\rho}^{ \pm}\right)} \mathbf{1}_{\vec{\xi} \notin \mathcal{X}_{C_{0}}^{ \pm}}}{\sum_{\vec{\xi}_{C_{0}}} \prod_{x \in C_{0}} e^{\beta \mathfrak{J}_{\gamma}\left(\vec{\xi}_{x} \cdot \vec{\rho}^{ \pm}\right)}} \\
& \approx \exp \left\{-\beta\left(\inf _{\vec{\rho}_{C_{0}} \notin \mathcal{X}_{C_{0}}^{ \pm}} \mathcal{F}_{\beta, C_{0}}^{(1)}\left(\vec{\rho}_{C_{0}}\right)-\inf _{\vec{\rho}_{C_{0}}} \mathcal{F}_{\beta, C_{0}}^{(1)}\left(\vec{\rho}_{C_{0}}\right)\right)\right\}
\end{aligned}
$$

Following the analysis of section $[$, one gets a similar estimate for the large deviation cost as

$$
\inf _{\vec{\rho}_{C_{0}} \notin \mathcal{X}_{C_{0}}^{ \pm}} \mathcal{F}_{\beta, C_{0}}^{(1)}\left(\vec{\rho}_{C_{0}}\right)-\inf _{\vec{\rho}_{C_{0}}} \mathcal{F}_{\beta, C_{0}}^{(1)}\left(\vec{\rho}_{C_{0}}\right) \geq c \gamma^{2 a}\left|C^{\ell,-}\right|
$$

for some constant $c$ and $\gamma$ small enough. The estimate (D.5) then follows.

\section{Appendix E. Proof of (7.57)-(7.58)}

In this appendix we prove that for any $\psi_{B} \in \mathcal{B}^{0}$ there is $\vec{\psi}_{B}^{*}$ :

$$
\vec{\psi}_{B}^{*}= \begin{cases}\vec{\psi}_{B} & \text { on } \delta_{\text {in }}^{\ell_{+} / 4}[B] \\ \vec{\rho}^{ \pm} & \text {elsewhere. }\end{cases}
$$

so that:

$$
\mathcal{F}_{\gamma, B, u}^{\mathrm{eff}}\left(\vec{\psi}_{B} \mid \vec{\xi}_{B^{c}}^{\left(\ell_{0}\right)}\right) \geq \mathcal{F}_{\gamma, B, u}^{\mathrm{eff}}\left(\vec{\psi}_{B}^{*} \mid \vec{\xi}_{B^{c}}^{\left(\ell_{0}\right)}\right)+c \gamma^{1 / 4}\left(\gamma^{1 / 8}|A|\right)
$$

The proof is analogous to the case of the Ising model widely analyzed in [26] to which we refer for details. A sketchy version is reported here for completeness.

Let $\Sigma$ as in (7.54), and

$$
\Delta=A \sqcup \delta_{\text {out }}^{\ell+, \gamma / 4}[A]
$$

Then, recalling that the interaction term appearing in the excess of free energy is always positive, we get a lower bound by neglecting the interaction between $\Delta$ and $B \backslash \Delta$ :

$$
\mathcal{F}_{\gamma, B, u}^{\mathrm{eff}}\left(\vec{\psi}_{B} \mid \vec{\xi}_{B^{c}}^{\left(\ell_{0}\right)}\right) \geq \mathcal{F}_{\gamma, B \backslash \Delta, u}^{\mathrm{eff}}\left(\vec{\psi}_{B \backslash \Delta} \mid \vec{\xi}_{B^{c}}^{\left(\ell_{0}\right)}\right)+\mathcal{F}_{\gamma, \Delta, u}^{\mathrm{eff}}\left(\vec{\psi}_{\Delta}\right)
$$

where, for any sets $D, F \sqsubset \mathbb{R}^{d}$ : 


$$
\begin{aligned}
& \mathcal{F}_{\gamma, D, u}^{\mathrm{eff}}\left(\vec{\psi}_{D}\right):=\int_{D} \Phi_{u}^{\mathrm{eff}, \pm}\left(\vec{\psi}_{D}\right)+\frac{u}{4} \int_{D \times D} J_{\gamma}\left(r, r^{\prime}\right)\left[\vec{\psi}_{D}(r)-\vec{\psi}_{D}\left(r^{\prime}\right)\right]^{2} \\
& \mathcal{F}_{\gamma, D, u}^{\mathrm{eff}}\left(\vec{\psi}_{D} \mid \vec{\xi}_{F}\right):=\int_{D} \Phi_{u}^{\mathrm{eff}, \pm}\left(\vec{\psi}_{D}\right)+\frac{u}{4} \int_{D \times D} J_{\gamma}\left(r, r^{\prime}\right)\left[\vec{\psi}_{D}(r)-\vec{\psi}_{D}\left(r^{\prime}\right)\right]^{2} \\
& +\frac{u}{2} \int_{D \times F} J_{\gamma}\left(r, r^{\prime}\right)\left[\vec{\psi}_{D}(r)-\vec{\xi}_{F}\left(r^{\prime}\right)\right]^{2}
\end{aligned}
$$

For any $\psi_{B} \in \mathcal{B}^{0}(\underline{(7.56)})$, since $\vec{\psi}_{B}^{*}(r) \equiv \vec{\psi}_{B}(r)$ on $r \in \delta_{\text {in }}^{\ell_{+}, \gamma} / 4[B] \sqcup \Sigma$, and $\vec{\psi}_{B}(r)=\vec{\rho}^{ \pm}$on $r \in \Sigma$, we have that:

$$
\mathcal{F}_{\gamma, B \backslash \Delta, u}^{\text {eff }}\left(\vec{\psi}_{B \backslash \Delta} \mid \vec{\xi}_{B^{c}}^{\left(\ell_{0}\right)}\right)=\mathcal{F}_{\gamma, B, u}^{\text {eff }}\left(\vec{\psi}_{B}^{*} \mid \vec{\xi}_{B^{c}}^{\left(\ell_{0}\right)}\right)
$$

in fact the distance between the sets $\delta_{\text {in }}^{\ell_{+, \gamma} / 4}[B]$ and $\Delta$ is larger than $\gamma^{-1}$ and $\mathcal{F}_{\gamma, \Delta, u}\left(\vec{\rho}^{ \pm}\right) \equiv 0$ :

Hence we need to prove that for any $\vec{\psi}_{B} \in \mathcal{B}^{0}$ :

$$
\mathcal{F}_{\gamma, \Delta, u}^{\mathrm{eff}}\left(\vec{\psi}_{\Delta}\right) \geq c \gamma^{1 / 4}\left(\gamma^{1 / 8}|A|\right)
$$

It is convenient here to fix a specific color $\hat{p}$ instead of distinguish only disordered and ordered configurations.

We then denote by

$$
\mathcal{S}^{\hat{p}}:=\left\{r \in \hat{A}:\left\|\vec{\psi}_{B}(r)-\vec{\rho}^{\hat{p}}\right\|_{\star} \geq \gamma^{1 / 8}\right\}
$$

that can be written as the sum of two sets $\mathcal{S}_{0}, \mathcal{S}_{1}$ :

$$
\begin{aligned}
& \mathcal{S}_{0}:=\left\{r \in \mathcal{S}:\left\|\vec{\psi}_{B}(r)-\vec{\rho}^{\hat{p}}\right\|_{\star} \geq \gamma^{1 / 8} \forall \hat{q}=-1,1, \ldots, Q\right\} \\
& \mathcal{S}_{1}:=\quad\left\{r \in \mathcal{S}: \exists \hat{q} \neq \hat{p}:\left\|\vec{\psi}_{B}(r)-\vec{\rho}^{\hat{q}}\right\|_{\star} \leq \gamma^{1 / 8}\right\}
\end{aligned}
$$

Recalling the definition of $\Phi_{u}^{\text {eff, }, \hat{p}}(\vec{v})$ in (7.52), and (7.49)-(17.51), we will prove that there are positive constants, $c_{0}, c_{1}, c_{2}$, so that:

$$
\begin{aligned}
& \int_{\mathcal{S}_{0}} \Phi_{u}^{\mathrm{eff}, \hat{p}}(\vec{\psi}(r)) \geq c_{0} \gamma^{1 / 4}\left|\mathcal{S}_{0}\right| \\
& \int_{\mathcal{S}_{1}} \Phi_{u}^{\mathrm{eff}, \hat{p}}(\vec{\psi}(r)) \geq\left(c_{1} u+c_{2}(1-u)\right)\left|\mathcal{S}_{1}\right|
\end{aligned}
$$

(E.4) and (E.5) prove (E.3) 
Proof of (E.4). (E.4) follows from the bound immediately obtained by the explicit expression of $\Phi_{u}^{\text {eff, }} \hat{p}(\vec{v})$ :

$$
\inf _{u \in(0,1)} \inf _{\left\{\vec{v}:\left\|\vec{v}-\rho^{\hat{q}}\right\|_{\star} \geq \gamma^{1 / 8} \forall \hat{q}\right\}} \Phi_{u}^{\mathrm{eff}, \hat{p}}(\vec{v}) \geq c_{0} \gamma^{1 / 4}
$$

$c_{0}$ a suitable constant

Proof of (E.5). Suppose $\left\|\vec{v}-\bar{\rho}^{\hat{q}}\right\|_{\star} \leq \gamma^{1 / 8}$ for some $\hat{q} \neq \hat{p}$. We will prove separately two bounds:

$$
\begin{aligned}
& \int_{\mathcal{S}_{1}} \Phi_{u}^{\mathrm{eff}, \hat{p}}(\vec{\psi}(r)) \geq 2 c_{2}(1-u)\left|\mathcal{S}_{1}\right| \\
& \int_{\mathcal{S}_{1}} \Phi_{u}^{\mathrm{eff}, \hat{p}}(\vec{\psi}(r)) \geq 2 c_{1} u\left|\mathcal{S}_{1}\right|
\end{aligned}
$$

that together give E.5

Proof of E.6

$$
\Phi_{u}^{\mathrm{eff}, \hat{p}}(\vec{v}) \geq(1-u)\left[-\vec{\rho}^{\hat{p}} \cdot\left(\vec{v}-\vec{\rho}^{\hat{p}}\right)+\frac{1}{\beta}\left[\vec{v} \ln \vec{v}-\vec{\rho}^{\hat{p}} \ln \vec{\rho}^{\hat{p}}\right]\right] \geq 0
$$

Since $\vec{\rho}^{\hat{p}}$ is a solution of the mean field equations $\vec{\rho}^{\hat{p}}=\frac{e^{\beta \vec{p}^{\hat{p}}}}{\sum_{i} e^{\beta \rho_{i}^{\hat{p}}}}$, it satisfies:

$$
\beta \rho_{i}^{\hat{p}}=\ln \rho_{i}^{\hat{p}}-\ln C
$$

with $C=\sum_{i} e^{\beta \rho_{i}^{\hat{p}}}$ and the square parenthesis in r.h.s. of (E.8), can be rewritten as:

$$
\begin{aligned}
& {\left[-\vec{\rho}^{\hat{p}} \cdot\left(\vec{v}-\vec{\rho}^{\hat{p}}\right)+\frac{1}{\beta}\left[\vec{v} \ln \vec{v}-\vec{\rho}^{\hat{p}} \ln \vec{\rho}^{\hat{p}}\right]\right]} \\
& \quad=-\frac{1}{\beta} \sum_{i}\left(v_{i}-\rho_{i}^{\hat{p}}\right) \ln \rho_{i}^{p}+\frac{1}{\beta} \sum_{i}\left(v_{i}-\rho_{i}^{\hat{p}}\right) \ln C^{-1}+\frac{1}{\beta}\left[\vec{v} \ln \vec{v}-\vec{\rho}^{\hat{p}} \ln \vec{\rho}^{\hat{p}}\right] \\
& \quad=-\frac{1}{\beta} \sum_{i}\left(v_{i}-\rho_{i}^{\hat{p}}\right) \ln \rho_{i}^{p}+\frac{1}{\beta}\left[\vec{v} \ln \vec{v}-\vec{\rho}^{\hat{p}} \ln \vec{\rho}^{\hat{p}}\right]
\end{aligned}
$$

where in the last equality we used the fact that $\sum_{i} v_{i}=\sum_{i} \rho_{i}^{\hat{p}}=1$. We then have:

$$
\Phi_{u}^{\text {eff }, \hat{p}}(\vec{v}) \geq \frac{(1-u)}{\beta} \vec{v} \ln \frac{\vec{v}}{\vec{\rho}^{\hat{p}}}
$$

and by Kullback-Leibler inequality:

$$
\begin{aligned}
\Phi_{u}^{\mathrm{eff}, \hat{p}}(\vec{v}) & \geq \frac{(1-u)}{2 \beta}\left(\vec{v}-\vec{\rho}^{\hat{p}}\right)^{2} \\
& \geq \frac{(1-u)}{2 \beta}\left[\left(\vec{\rho}^{\hat{q}}-\vec{\rho}^{\hat{p}}\right)^{2}-\gamma^{1 / 4}\right]
\end{aligned}
$$

We consider separately the case when $\hat{q}=-1, \hat{p}>0$ (or viceversa) and the case where both $\hat{q}, \hat{p}$ are positive. 
In the first case:

$$
\begin{aligned}
\Phi_{u}^{\mathrm{eff}, \hat{p}}(\vec{v}) & \geq \frac{(1-u)}{2 \beta}\left[\left(\rho_{A}-\frac{1}{Q}\right)^{2}+\left(\rho_{B}-\frac{1}{Q}\right)^{2}(Q-1)-\gamma^{1 / 4}\right] \\
& \geq \frac{(1-u)}{2 \beta}\left[\frac{Q(1-2 / Q)^{2}}{(Q-1)}-\gamma^{1 / 4}\right]
\end{aligned}
$$

If both $\hat{q}, \hat{p}$ are positive

$$
\begin{aligned}
\Phi_{u}^{\mathrm{eff}, \hat{p}}(\vec{v}) & \geq \frac{(1-u)}{2 \beta}\left[2\left(\rho_{A}-\rho_{B}\right)^{2}-\gamma^{1 / 4}\right] \\
& \geq \frac{(1-u)}{2 \beta}\left[\left(\frac{Q(1-2 / Q)}{(Q-1)}\right)^{2}-\gamma^{1 / 4}\right]
\end{aligned}
$$

Finally, for $\gamma$ small enough:

$$
\Phi_{u}^{\mathrm{eff}, \hat{p}}(\vec{v}) \geq \frac{(1-u)}{20 \beta}
$$

We now prove (E.7). Let $r \in \mathcal{S}_{1}$

$$
\int d r^{\prime} J_{\gamma}\left(r, r^{\prime}\right)\left(\vec{\psi}_{B}(r)-\vec{\psi}_{B}\left(r^{\prime}\right)\right)^{2} \geq \int d r^{\prime} J_{\gamma}^{\left(\ell_{-, \gamma}\right)}\left(r,\left\langle r^{\prime}\right\rangle\right)\left(\vec{\psi}_{B}(r)-\vec{\psi}_{B}\left(r^{\prime}\right)\right)^{2}-c \gamma \ell_{-, \gamma}
$$

where

$$
J_{\gamma}^{\left(\ell_{-, \gamma}\right)}\left(r,\left\langle r^{\prime}\right\rangle\right):=\frac{1}{\mid C^{\ell_{-, \gamma} \mid}} \int_{C_{r^{\prime}}^{\ell_{-}, \gamma}} J_{\gamma}\left(r, r^{\prime \prime}\right) d r^{\prime \prime}
$$

and it is constant on the cubes of the partition $\mathcal{D}^{\ell_{-, \gamma}}$. By Cauchy-Schwartz inequality:

$$
\begin{aligned}
& \frac{1}{\mid C^{\ell_{-, \gamma} \mid}} \int_{C_{r^{\prime}}^{\ell_{-, \gamma}}} d r^{\prime} J_{\gamma}^{\left(\ell_{-, \gamma}\right)}\left(r,\left\langle r^{\prime}\right\rangle\right)\left(\vec{\psi}_{B}(r)-\vec{\psi}_{B}\left(r^{\prime}\right)\right)^{2} \\
& \quad \geq J_{\gamma}^{\left(\ell_{-, \gamma}\right)}\left(r,\left\langle r^{\prime}\right\rangle\right)\left(\vec{\psi}_{B}(r)-\frac{1}{\mid C^{\ell_{-, \gamma} \mid}} \int_{C_{r^{\prime}}^{\ell_{-, \gamma}}} \vec{\psi}_{B}\left(r^{\prime \prime}\right) d r^{\prime \prime}\right)^{2} \\
& \quad \geq J_{\gamma}^{\left(\ell_{-, \gamma}\right)}\left(r,\left\langle r^{\prime}\right\rangle\right)\left(\left(\vec{\rho}^{\hat{q}}-\vec{\rho}^{\hat{p}}\right)^{2}-\gamma^{1 / 4}-\gamma^{2 a}\right)
\end{aligned}
$$

if both $\hat{q}, \hat{p}>0,\left(\vec{\rho}^{\hat{q}}-\vec{\rho}^{\hat{p}}\right)^{2}=2\left(\rho_{A}-\rho_{B}\right)^{2}=2\left(\frac{Q(1-2 / Q)}{Q-1}\right)^{2}$. While if $\hat{q}$ or $\hat{p}$ is equal to -1 : $\left(\vec{\rho}^{\hat{q}}-\vec{\rho}^{\hat{p}}\right)^{2}=2(1-2 / Q)^{2}$ Then:

$$
\int d r^{\prime} J_{\gamma}\left(r, r^{\prime}\right)\left(\vec{\psi}_{B}(r)-\vec{\psi}_{B}\left(r^{\prime}\right)\right)^{2} \geq \frac{1}{5}-c \gamma \ell_{-, \gamma}-\gamma^{1 / 4}-\gamma^{2 a}
$$

and

$$
\frac{u}{4} \int_{\mathcal{S}_{1}} d r d r^{\prime} J_{\gamma}\left(r, r^{\prime}\right)\left(\vec{\psi}_{B}(r)-\vec{\psi}_{B}\left(r^{\prime}\right)\right)^{2} \geq\left(\frac{1}{30}\right) u\left|\mathcal{S}_{1}\right|
$$




\section{Appendix F. Proof of Theorem 6.1}

(6.2) follows from (4.6) and (6.3), by setting

$$
e^{-\beta \mathcal{H}_{\Lambda}^{\hat{p}}\left(\vec{\xi}_{\Lambda}\right)}=\sum_{\underline{\Gamma} \in \mathcal{B}_{\Lambda}^{+}} W^{\hat{p}}\left(\underline{\Gamma}, \vec{\xi}_{\Lambda}\right)
$$

To prove the remaining statements we use a cluster expansion to express the energy $\mathcal{H}_{\Lambda}^{\hat{p}}\left(\vec{\xi}_{\Lambda}\right)$ in terms of a sum of weights of polymers, which will then identify the many-body potentials $U_{\Delta}^{\hat{p}}\left(\vec{\xi}_{\Delta}\right)$.

Polymers are functions $I:\{\Gamma\}^{\hat{p}} \rightarrow \mathbb{N}_{+}$such that the collection $\{\Gamma: I(\Gamma)>0\}$ is finite and connected, where two elements $\Gamma$ and $\Gamma^{\prime}$ in $\{\Gamma\}^{\hat{p}}$ are connected if $\overline{\operatorname{sp}(\Gamma)} \sqcap \overline{\operatorname{sp}\left(\Gamma^{\prime}\right)} \neq \emptyset$. Denote by $\mathcal{P}^{\hat{p}}$ the collection of all polymers and by $\mathcal{P}_{\Lambda}^{\hat{p}}$ those made by contours in $\{\Gamma\}_{\Lambda}^{\hat{p}}$. It then follows from Kotecký and Preiss, [20, that, if the Peierls constant is large enough, there are numbers $\varpi(I, \underline{s})$, such that

$$
\ln \sum_{\underline{\Gamma} \in \mathcal{B}_{\Lambda}^{\hat{p}}} W^{\hat{p}}(\underline{\Gamma}, \vec{\xi})=\sum_{I \in \mathcal{P}_{\Lambda}^{\hat{p}}} \varpi^{\hat{p}}(I, \vec{\xi})
$$

Calling $\operatorname{sp}_{+}(I)=\bigsqcup_{\Gamma: I(\Gamma)>0} \operatorname{sp}_{+}(\Gamma)$, with $\operatorname{sp}_{+}(\Gamma):=\operatorname{sp}(\Gamma) \sqcup \delta_{\text {out }}^{\ell_{+}, \gamma}[\operatorname{sp}(\Gamma)]$, we then set:

$$
U_{\Delta}^{\hat{p}}\left(\vec{\xi}_{\Delta}\right)=-\frac{1}{\beta} \sum_{I \in \mathcal{P}_{\Lambda}^{\hat{p}}, \mathrm{sp}_{+}(I)=\Delta} \varpi^{\hat{p}}\left(I, \vec{\xi}_{\Delta}\right)
$$

$\varpi^{\hat{p}}(I, \vec{\xi})$ satisfy the bound:

$$
\sum_{I: \operatorname{sp}(I) \ni x}\left\|\varpi^{\hat{p}}(I, \vec{\xi})\right\|_{\infty}\left\{\prod_{\Gamma: I(\Gamma)>0} e^{\frac{\hat{\kappa}_{\gamma}}{2} N_{\Gamma} I(\Gamma)}\right\}<1
$$

(F.12) follows by the general theory (see [20]) after noting that the number of contours $\#(\Delta):=\#\{\Gamma: \operatorname{sp}(\Gamma)=\Delta\}$ is bounded by $(Q+1)^{N \Delta \gamma^{-2 \alpha d}}$ and for $\gamma$ small enough, since $2 \alpha \ll 1, \#(\Delta) e^{-\mathfrak{K}_{\gamma} N_{\Delta}} \ll 1,\left(\mathfrak{K}_{\gamma} / 2\right.$ is not optimal). (6.6) and (6.7) then follows from (F.11)-(F.12):

$$
\begin{aligned}
\beta \sum_{\Delta \ni x}\left|U_{\Delta}^{\hat{p}}\left(\vec{\xi}_{\Delta}\right)\right| & \leq \sum_{I: \mathrm{sp}_{+}(I) \ni x}\left\|\varpi^{\hat{p}}(I, \vec{\xi})\right\|_{\infty} \\
& \leq \sum_{I: \mathrm{sp}_{+}(I) \ni C_{x}}\left\|\varpi^{\hat{p}}(I, \vec{\xi})\right\|_{\infty}\left\{\prod_{\Gamma: I(\Gamma)>0} e^{\frac{\hat{\kappa}_{\gamma}}{2}\left(N_{\Gamma} I(\Gamma)-N_{\Gamma}\right)}\right\}
\end{aligned}
$$

Where we used the fact that $N_{\Gamma} I(\Gamma)-N_{\Gamma} \geq 0$. Since $\min _{\Gamma} N_{\Gamma}=3^{d}$, then:

$$
\begin{aligned}
\beta \sum_{\Delta \ni x}\left|U_{\Delta}^{\hat{p}}\left(\vec{\xi}_{\Delta}\right)\right| & \leq e^{-\frac{\mathfrak{K}_{\gamma}}{2} 3^{d}} \sum_{C \in\left[C_{x} \sqcup \delta_{\text {out }}^{\ell+\gamma}\left[C_{x}\right]\right]} \sum_{I: \mathrm{sp}_{+}(I) \sqsupset C}\left\|\varpi^{\hat{p}}(I, \vec{\xi})\right\|_{\infty}\left\{\prod_{\Gamma: I(\Gamma)>0} e^{\frac{\mathfrak{K}_{\gamma}}{2} N_{\Gamma} I(\Gamma)}\right\} \\
& \leq 3^{d} e^{-\frac{\mathfrak{K}_{\gamma}}{2} 3^{d}} .
\end{aligned}
$$


last inequality uses the (F.12) and the translation invariance of $U^{\hat{p}}$, for $\gamma$ small enough (6.6) follows. (6.7) can be proven analogously. The proof of (6.10) follows from (4.9) in a similar way. Theorem 6.1 is proved.

\section{References}

[1] M. Aizenman, J. T. Chayes, L Chayes, C. M. Newman: Discontinuity of the magnetization in onedimensional $\frac{1}{|x-y|^{2}}$ Ising and Potts models, J. Stat. Phys. 50 1-40, (1988)

[2] F. Baffioni, T. Kuna, I. Merola, E. Presutti: A liquid vapor phase transition in quantum statistical mechanics, Submitted to Memoirs AMS (2004)

[3] F. Baffioni, T. Kuna, I. Merola, E. Presutti: A relativized Dobrushin uniqueness condition and applications to Pirogov-Sinai models, Submitted to Memoirs AMS (2004)

[4] R. J. Baxter: Potts model at the critical temperature, J. Phys. C 6, L445 (1973)

[5] R. J. Baxter, H. N. V. Temperley, S. E. Ashley: Triangular Potts model at its transition temperature, and related models, Proc. Roy. Soc. London, Ser. A 358, 535 (1978)

[6] R. J. Baxter: Exactly solved sodels in statistical mechanics, Academic Press, London (1982).

[7] M.Biskup, L. Chayes: Rigorous analysis of discontinous phase transitions via mean field bounds, Commun. Math. Phys. 238, 53-93 (2003)

[8] M.Biskup, L. Chayes, N.Crawford: Mean-field driven first order phase transitions in systems with longrange interactions, Submitted to J. Stat. Phys.(2005)

[9] A. Bovier, I. Merola, E. Presutti, M. Zahradnik: On the Gibbs phase rule in the Pirogov-Sinai regime, J. Stat. Phys. 114, 1235-1267 (2004)

[10] A. Bovier, M. Zahradnik: The low-temperature phase of Kac-Ising models, J. Stat. Phys. 87, 311-332 (1997)

[11] A. Bovier,M. Zahradnik: Cluster expansions and Pirogov-Sinai theory for long range spin systems, Markov Process and Related Fields 8, 443-478 (2002)

[12] J. Bricmont, K. Kuroda, JL Lebowitz, First order phase transitions in lattice and continuous systems: Extension of Pirogov-Sinai theory, Commun. Math. Phys. 101:501 (1985).

[13] M. Cassandro, E. Presutti: Phase transitions in Ising systems with long but finite range, Markov Processes and Related Fields 2, 241-262 (1996)

[14] C. M. Fortuin, P. W. Kasteleyn: On the random cluster model I: introduction and relation to other models, Physica 57, 536-564 (1972) 
[15] H. Georgii, O. Häggström, C. Maes The Random Geometry of Equilibrium Phases, Phase Transitions and Critical Phenomena, vol 18, Eds C. Domb and J.L. Lebowitz (Academic Press, London) pp 1-142.

[16] G. R. Grimmett: The stochastic random cluster process and the uniqueness of random cluster measures, Ann. Prob. 23, 1461-1510 (1995).

[17] G. R. Grimmett: The random-cluster model, Probability, Statistics and Optimization, F.P.Kelly ed., John Wiley \& Sons, Chichester, 49-63 (1994)

[18] H. Kesten, R. H. Schonmann: Behavior in large dimension of the Potts and Heisenberg models, Reviews in Math. Phys. 1, 147-182 (1990)

[19] R. Kotecký, S. B. Shlosman: First order phase transition in large entropy lattice models, Commun. Math. Phys. 83, 493-515 (1982)

[20] R. Kotecký, D. Preiss: Cluster expansion for abstract polymer models Comm. Math. Phys., 103, 491-498 (1986)

[21] J. L. Lebowitz, A. E. Mazel and E. Presutti: Liquid-vapour phase transitions for systems with finite range interactions, J. Stat. Phys. 94, 955-1025 (1999)

[22] J. L. Lebowitz, A. E. Mazel, E. Presutti: Rigorous proof of a liquid-vapour phase transition in a continuum particle system Phys. Rev. Letters 80, 4701 (1998)

[23] J. L. Lebowitz and O. Penrose: Rigorous treatment of the Van der Waals Maxwell theory of the liquidvapor transition J. Math. Phys. 7, 98-113 (1966)

[24] S.A. Pirogov, Ya. G. Sinai: (i) Teor. Mat. Fiz. 25, 358-369, (1975), in Russian; English translation: Phase diagrams of classical lattice systems, Theor. Math. Phys. 25 1185-1192, (1975). (ii) Teor. Mat. Fiz. 26, 61-76, (1976), in Russian; English translation: Phase diagrams of classical lattice systems. Continuation, Theor. Math. Phys. 26 39-49, (1976)

[25] R. Potts: Some generalyzed order-disorder transformations Proc. Camb. Phil. Soc. 48, 106-109 (1952)

[26] E. Presutti: Scaling limits in statistical mechanics $\&$ microstructures in continuum mechanics. In preparation (2005)

[27] S. Reynal, H. T. Diep: Reexamination of the long-range Potts model: A multicanonical approach Phys. Rev E69 026109 (2004)

[28] A. D. Sokal: Chromatic polynomials, Potts models and all that, Physica A279, 324-332 (2000)

[29] D.J.A. Welsh and C. Merino, The Potts model and the Tutte polynomial,Journal of Mathematical Physics 41(3), 2000, 11271149.

[30] F. Y. Wu: The Potts model, R. Mod. Phys. 54, 235-268 (1982) 
[31] M. Zahradnì: A short course on the Pirogov-Sinai theory, Rend. Mat. Appl 18, 411-486 (1998)

Thierry Gobron, CNRS-UMR 8089, Laboratoire de Physique théorique et modélisation, Université de Cergy-Pontoise, France

E-mail address: gobron@ptm.u-cergy.fr

Immacolata Merola, Dipartimento di matematica pura e applicata, Università dell'Aquila, ITALY

E-mail address: merola@univaq.it 